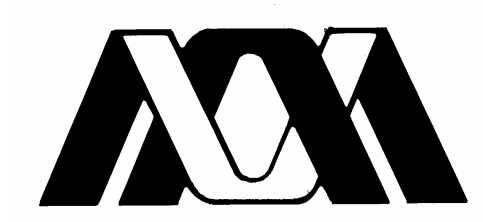

Casa abierta al tiempo

\author{
UNIVERSIDAD AUTÓNOMA METROPOLITANA \\ UNIDAD IZTAPALAPA
}

DIVISIÓN DE CIENCIAS BÁSICAS E INGENIERIA

CONSERVATIVIDAD DE LA SOLUCIÓN MINIMAL DE LA ECUACION DE LINDBLAD

TESIS QUE PARA OBTENER EL GRADO DE

MAESTRO EN CIENCIAS (MATEMATICAS)

PRESENTA

MAXIMINO CRUZ MARTINEZ

DIRECTOR DE TESIS

DR. JULIO CESAR GARCIA CORTE

México, D.F., A 19 DE MARZO DE 2004. 


\section{$\Lambda \mathbf{U}$ Universidad Autónoma Metropolitana- Iztapalapa. División de Ciencias Básicas e Ingeniería.}

\section{"CONSERVATIVIDAD DE LA SOLUCIÓN MINIMAL DE LA ECUACIÓN DE LINDBLAD"}

Tesis que para obtener el grado de Maestro en Ciencias (Matemáticas) presenta:

Maximino Cruz Martínez

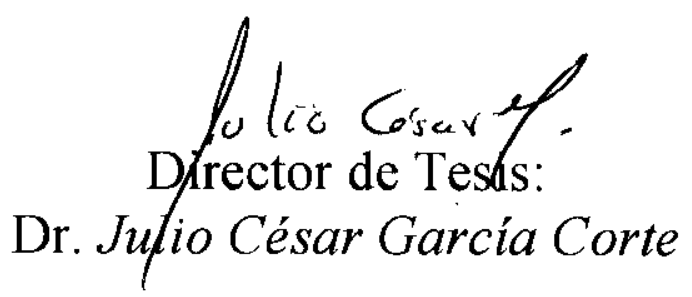

México. D. F. a 19 de Marzo de 2004 


\section{$\Lambda \mathbf{U}$ Universidad Autónoma Metropolitana- Iztapalapa. División de Ciencias Básicas e Ingeniería.}

\section{"CONSERVATIVIDAD DE LA SOLUCIÓN MINIMAL DE LA ECUACIÓN DE LINDBLAD "}

Tesis que para obtener el grado de Maestro en Ciencias (Matemáticas) presenta:

Maximino Cruz Martínez

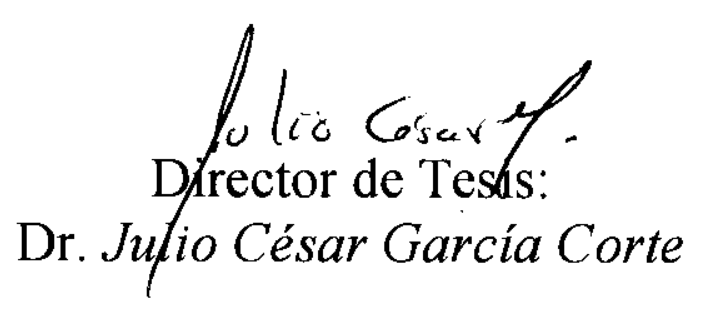

México. D. F. a 19 de Marzo de 2004 


\section{AGRADECIMIENTOS}

El presente trabajo está dedicado a las siguientes

personas:

\section{Mi hermano:}

Tiburcio Cruz Martínez.

Gracias a su apoyo económico incondicional, fue posible que concluyese la maestría.

\section{Dr. Julio Cesar García Corte.}

Investigador de tiempo completo, en el área de probabilidad, U. A. M. I. Gracias a su ardua labor e interés inagotable por el trabajo fue posible que éste se concluyese con éxito.

A los doctores :

Dr. Jorge A. León Vázquez.

Dr. Roberto Quezada Batalla.

Dr. Juan Ruíz de Chavez Somosa.

Por aceptar ser mis sinodales.

Mis padres:

Angel Cruz Arias.

Juana Martínez, Ramírez.

Mis hermanos:

Aurelio, Herminia, Angel Jr. Matilde y Mariana.

Mis sobrinos:

Edi y Griselda.

Todos ellos aun en la distancia, nunca dejaron de apoyarme moralmente.

vfil eject 


\section{INTRODUCCION}

El propósito del presente trabajo es exponer la teoría de los semigrupos dinámicos cuánticos (S.D.C.). Los resultados que se exponen se encuentran en [8], aquí los hemos desarrollado incluyendo sus respectivos detalles con al intención de que este trabajo sirva de motivación y apoyo a otros sobre esta misma área de investigación. La proposición 3.2.11 es una extensión de la proposición 3.33 pág 64 de [8] y se demuestra por primera vez en [12]. En el primer capítulo se exponen los antecedentes necesarios para comprender en qué consiste la teoría de los semigrupos dinámicos cuánticos. Habrá quizás uno que otro resultado que no esté demostrado en esta primera parte, pero se incluyen referencias donde el lector podrá consultar las demostraciones necesarias.

Un problema importante de esta teoría es determinar cuándo un semigrupo dinámico cuántico (S.D.C.) es conservativo. El semigrupo dinámico cuántico minimal (S.D.C.M.)) que en este trabajo se construye en capítulo 3 a partir de un semigrupo de contracciones y cuando éste es conservativo, es decir, preserva a el operador identidad, es la única solución a la ecuación de Lindblad, ecuación (3.6) Capítulo 3, pág. 49 de este trabajo. También en el capítulo 3 se dan condiciones necesarias y suficientes para la conservatividad del S.D.C.M. Posteriormente en el capítulo 4 bajo la hipótesis AA y C, (págs. 69-84) se desarrollan los principales criterios (condiciones suficientes) que garantizan la conservatividad del S.D.C.M. Por último en el capítulo 5 se exponen ejemplos en los cuales aplicamos la teoría desarrollada 


\section{Índice general}

1. Preliminares 5

1.1. El espacio $\varnothing(h) \ldots \ldots \ldots \ldots \ldots$

1.2. Topologías en $B(h) \ldots \ldots \ldots$

2. Semigrupos dinámicos cuánticos(S.D.C.) 23 2.1 .

3. El Semigrupo Dinámico Cuántico M inimal (S.D.C.M.) 41

3.1. Hipótesis A . . . . . . . . . . . . . . . . . . . 42

3.2. El Resolvente del Semigrupo Minimal . . . . . . . . . . . 63

4. Criterios de Conservatividad 81

4.1. Hipótesis C . . . . . . . . . . . . . . . . . . . . . 82

5. Ejemplos de S. D. C. 99

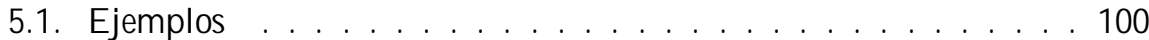
Bibliografía 
ÍNDICE GENERAL 


\section{Capítulo 1}

\section{Preliminares}

En este capítulo desarrollamos los prerrequisitos necesarios para el resto del trabajo. A unque no demostramos todos los resultados aquí enunciados indicaremos las referencias donde el lector podrá encontrar más detalles y las demostraciones correspondientes. 
De..nición 1.0.1 Sea U un espacio vectorial con coe..cientes en los complejos C. El espacio U es Ilamado un \$lgebra si existe una operación tal que a cada par de elementos a; b2 U se le asocia un elemento denotado por ab: Esta operación tiene las siguientes propiedades.

1) $a(b c)=(a b) c$

2) $a(b+c)=a b+a c$

3) $Æ(a b)=($ Ea) $b=a(A b)$, con $Æ 2 C$.

EI álgebra $U$ es Ilamada conmutativa 0 abeliana si $a b=$ ba; para todo a; b2 U:

De..nición 1.0.2 El álgebra $U$ es normada si a cada elemento a 2 U hay asociado un número real positivo jjajj; donde jj:jj satiface:

1) jjajj , 0; y jjajj $=0$ si y sólo si $a=0$ :

2) jjabj · jjajj jjbj:

3) jja + bjj · jjajj + jjbj:

4) $\mathrm{jj} /$ Æajj $=\mathrm{j}$ /f jjajj; con $Æ 2$ C.

De..nición 1.0.3 Una ææ-\$lgebra es una álgebra compleja U con una involución denotada por * con las siguientes propiedades:

$$
\begin{array}{ll}
\left(a^{\alpha}\right)^{\infty} & =a: \\
(a b)^{\alpha} & =b^{\alpha} a^{\alpha}: \\
(\Pi a+\pi b)^{\alpha} & =\Pi^{\alpha}+\hbar b^{\alpha} ;
\end{array}
$$

donde Пу п 2 C.

De..nición 1.0.4 U na x-\$lgebra U normada completa con la propiedad jja ${ }^{x} \mathrm{jj}=$ jjajj, para a 2 U; se Ilama*-álgebra de Banach.

De..nición 1.0.5 U na C*-álgebra es una xxálgebra U de B anach con la propiedad de que para todo a $2 \mathrm{U}$ jjaa ${ }^{\mathrm{a} j \mathrm{j}}=\mathrm{jjajj}^{2}$ : 
Ejemplo 1.0.6 Sea $\mathrm{h}$ un espacio de Hilbert y denotemos por B (h) el espacio de todos los operadores acotados en h: Las operaciones de suma y productos de elementos de $B(h)$ son las usuales. La norma de operadores $\mathrm{jj}: \mathrm{j}_{1}$ en $B(\mathrm{~h})$ es la siguiente, sea Z 2 B (h)

$$
\mathrm{jjZ}_{\mathrm{j} j_{1}}=\operatorname{supfjjZ}(\mathrm{a}) \mathrm{jj}: \mathrm{a} 2 \mathrm{~h} ; \mathrm{jjajj}=1 \mathrm{~g}
$$

y la involución es el adjunto. Claramente se tiene que ( $B(h) ; j j: j j_{1}$ ) es una $C^{x}$ álgebra.

Ejemplo 1.0.7 Sea $X$ un espacio localmente compacto, $C_{0}(X ; C)$ el espacio de las funciones continuas sobre $X$ que se anulan en el in..nito, es decir dado $f$ $\mathrm{C}_{0}(\mathrm{X} ; \mathrm{C})$ y " $>0$ existe $\mathrm{K} 1 / 2 \mathrm{X}$ compacto tal que jf $(\mathrm{x}) \mathrm{j}<$ ", para todo $\times 2 \mathrm{~K}^{\mathrm{c}}$;el complemento de $\mathrm{K}$ : De..namos una norma en $\mathrm{C}_{0}(\mathrm{X} ; \mathrm{C})$ de la siguiente forma

$$
\mathrm{jjf}_{\mathrm{j} j_{1}}=\operatorname{supfjf}(\mathrm{x}) \mathrm{j}: \times 2 \times \mathrm{g} \text {; }
$$

como involución * tómese $f(x)^{\alpha}=\overline{f(x)}$. Así $C_{0}(X ; C)$ es una $C^{*}$-álgebra y además es conmutativa.

De..nición 1.0.8 Sea U un álgebra con identidad y a 2 U, el conjunto resolvente de a es:

$$
\Omega(a)=f \Pi 2 C: \Pi \text { i a es invertibleg }
$$

y el espectro de a es el complemento de $\Omega($ (a).

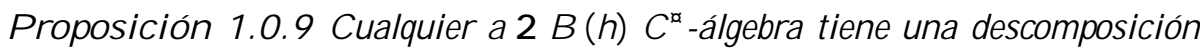
única en términos de elementos autoadjuntos $a_{1} y_{2} a_{2}$,

$$
a_{1}=\frac{a+a^{\infty}}{2} \quad \text { y } \quad a_{2}=\frac{a_{i} a^{\infty}}{2 i}
$$

donde $\mathrm{a}=\mathrm{a}_{1}+\mathrm{i} \mathrm{a}_{2}$. Además $\mathrm{a}_{1}$ y $\mathrm{a}_{2}$ se pueden expresar como combinación lineal de dos positivos.

\section{Demostración.}

Ver.[3], pág.38.

De..nición 1.0.10 Un *-mor..smo entre dos *-álgebras $U$ y $V$,es una tranformación lineal $\stackrel{0}{:} \mathrm{U} ! \mathrm{V}$ tal que:

1) $\cong\left(u_{i}\right)=0(u) \subseteq(i):$

2) $\circ\left(u^{a}\right)=(\underline{o} u)^{a}$

para todo $\mathrm{u} ; \mathrm{i} 2 \mathrm{U}$ :

Si además $\cong(I)=1$ entonces $\cong$ se llama *-homomor..smo. 
De..nición 1.0.11 Una representación de una $U C^{\circledR}$-álgebra es una pareja $(h ; \underline{\circ})$; donde $h$ es un espacio de Hilbert y $\varrho$ es un ${ }^{*}$-mor..smo de $U$ a $B(h)$ :

De..nición 1.0.12 Un elemento a $2 \cup$ C álgebra es llamado positivo(a , 0) si existe $b 2 U$ tal que $a=b^{a} b$; 0 si es combinación lineal con coe..cientes positivos de elementos de esta forma, y es estrictamente positivo si es distinto de cero.

De..nición 1.0.13 Sean $U, V$ dos $C^{\bowtie}$-álgebras y T: U ! V lineal, T es llamado $\mathrm{n}$ positivo si para cada $a_{1} ;: ; ; a_{n} 2 \mathrm{U}$ y $b_{1} ;:: ; b_{n} 2 \mathrm{~V}$ se tiene lo siguiente:

$$
x_{i ; j=1}^{n} b^{a} T\left(a_{i}^{a} a_{j}\right) b, 0:
$$

T es llamado positivo si es 1 positivo, es decir manda elementos positivos en positivos: $\mathrm{T}$ es Completamente $\mathrm{P}$ ositivo (CP) si es $\mathrm{n}$ positivo para cada $\mathrm{n}, 1$ :

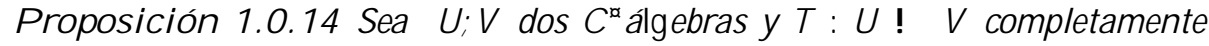
positivo entonces se cumple que

$$
\mathrm{T}\left(\mathrm{a}^{\alpha}\right)=\mathrm{T}(\mathrm{a})^{\alpha}:
$$

Demostración.

a) Sea $a, 0$ entonces $a=a^{\infty}$. Pero siendo $T C P$ se sigue que $T(a), 0$, de lo cual se deduce

$$
\mathrm{T}\left(\mathrm{a}^{\mathrm{a}}\right)=\mathrm{T}(\mathrm{a})=\mathrm{T}(\mathrm{a})^{\mathrm{a}}:
$$

b) Si a es autoadjunto entonces $a=a^{+}$i ai con $a^{+}$; $a^{i}, 0$ : De donde obtenemos que

$$
\begin{aligned}
\mathrm{T}\left(\mathrm{a}^{\mathrm{\alpha}}\right) & =\mathrm{T}^{\mathrm{i}} \mathrm{a}^{+}{ }^{\phi}{ }_{\mathrm{i}} \mathrm{T}^{\mathrm{i}}{ }_{\mathrm{a}^{\mathrm{i}}} \phi \\
& =\mathrm{T}^{\mathrm{i}} \mathrm{a}^{+} \phi_{\infty}{ }^{\mathrm{i}} \mathrm{T}^{\mathrm{i}} \mathrm{a}^{\mathrm{i}} \phi_{\infty} \\
& ={ }^{\mathrm{i}} \mathrm{T}^{\mathrm{i}} \mathrm{a}^{+} \phi^{\mathrm{i}} \mathrm{T}^{\mathrm{i}} \mathrm{a}^{\mathrm{i}} \phi \phi_{\infty} \\
& =\mathrm{T}(\mathrm{a})^{\infty}:
\end{aligned}
$$

c) Si a es arbitrario entonces

$$
a=\frac{a+a^{a}}{2}+i \frac{a i a^{a}}{2 i}
$$


donde $\frac{a+a^{\mathrm{x}}}{2} ; \frac{a_{i} a^{\mathrm{a}}}{2 \mathrm{i}}$ son autoadjuntos. Por lo tanto también se tiene que

$$
\begin{aligned}
T\left(a^{\infty}\right) & =T^{\mu} \frac{a+a^{x}}{2} i T^{\mu} \frac{a_{i} a^{x}}{2 i} \\
& =T \frac{\mu+a^{\infty}}{2}+i T \frac{a_{i} a^{x}}{2 i} \\
& =T(a)^{x}:
\end{aligned}
$$

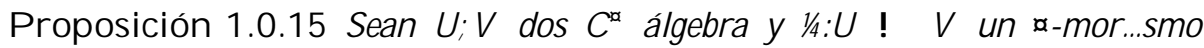
entonces $\stackrel{\circ}{ }$ es completamente positivo.

Demostración. Sea $n, 1, a_{1} ;:: ; a_{n} 2 \cup$ y $b_{1} ;:: ; b_{n} 2$ V

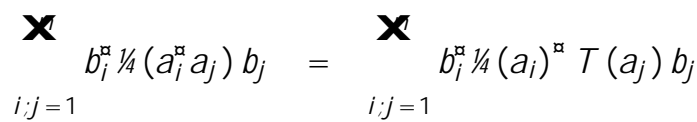

$$
\begin{aligned}
& =X^{n} b^{a_{0}}-\left(a_{i}\right)^{x} \underline{X^{n}} o\left(a_{j}\right) b
\end{aligned}
$$

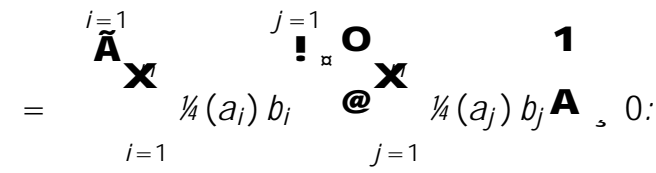

De..nición 1.0.16 a) Sea $U$ una $C^{a}$ álgebra. Para cada $n, 1$ denotamos por $\mathrm{U}-\mathrm{M}_{\mathrm{n}}$ a la álgebra de matrices $\mathrm{n} f \mathrm{n}$ con entradas en $\mathrm{U}$ : A sí cada $\times 2 \mathrm{U}-\mathrm{M}_{\mathrm{n}}$ es de la forma

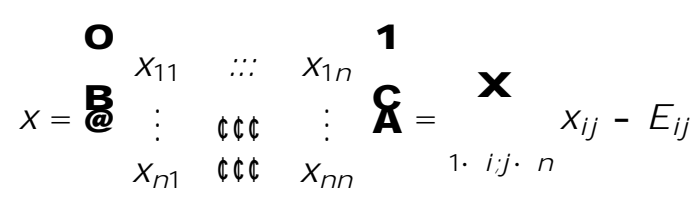

donde $E_{i j}$ es la matriz de $n \in n$ con ceros en todas sus entradas excepto en la ij -ésima entrada donde hay un 1: Las operaciones de suma, resta, multiplicación y tranpuesta conjugada de matrices en $U-M_{n}$ son las usuales en el álgebra lineal. Sólo que aquí las entradas son elementos de la $C^{\text {×álgebra }}$ $U$.

b) Sea $\mathrm{V}$ otra $\mathrm{C}$ álgebra y $\mathrm{T}: \mathrm{U}$ ! $\mathrm{V}$ una transformación lineal. Para cada $\mathrm{n} 2 \mathrm{~N} ; \mathrm{T}$ induce un operador lineal $T_{n}: U-M_{n}$ ! $V-M_{n}$ dado por,

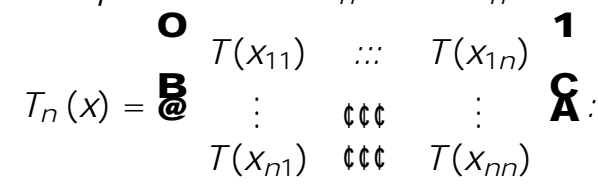


Proposición 1.0.17 Sea $U$ una $C^{\star}$ álgebra contenida en $B(h)$ para algún espacio de Hilbert $h$ y $\times 2 U-M_{n}$. Las siguientes condiciones son equivalentes:

1) $x$ es positivo ;

2) x es una suma ..nita de matrices de la forma

$$
\begin{aligned}
& a_{1}^{x} a_{1} \quad \pitchfork \notin \varnothing \quad a_{1}^{x} a_{n}
\end{aligned}
$$

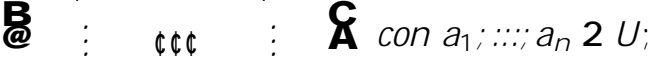

$$
\begin{aligned}
& a_{n}^{a} a_{1} \quad \phi \pitchfork \varnothing \quad a_{n}^{a} a_{n}
\end{aligned}
$$

3) Para todo $a_{1} ;: \ldots ; a_{n} 2 \cup$ se tiene que ${ }^{P} 1 \cdot i ; j \cdot n a_{i}^{\alpha} x_{i j} a_{j}, 0$ :

Demostración. Seguimos [8], pág.18.

1) implica 2)

Como $x$ es positivo entonces $x=y^{x} y$ con y $2 U-M_{n}$;

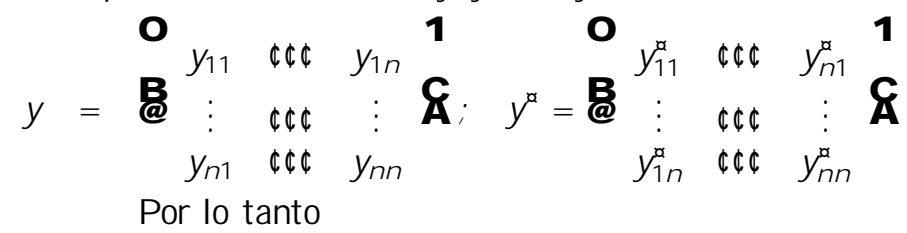

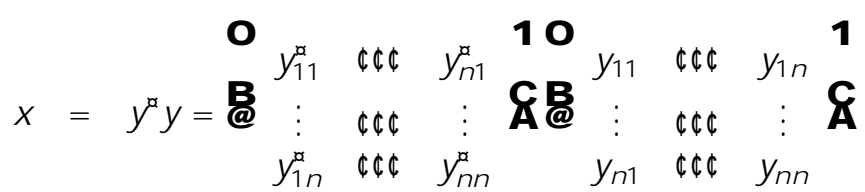

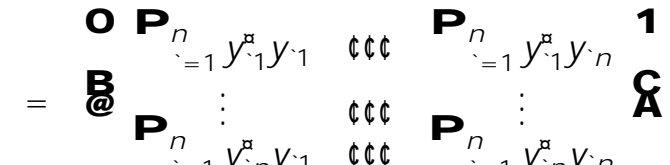

$$
\begin{aligned}
& 0=1 y_{n} y_{1}
\end{aligned}
$$

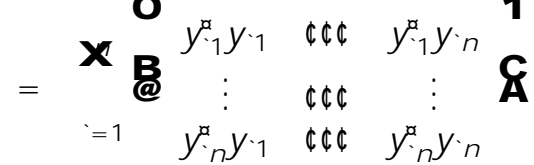


2) implica 3)

Como $x$ tipene la representación dado por 2) entonces claramente se tiene que $x_{i j}={ }^{n}=1 y_{i}^{a} y_{j}$; con lo cual

$$
\begin{aligned}
& X^{n} \quad A_{4} x_{i j} \\
& \mathrm{i} ; \mathrm{j}=1 \\
& =X_{i ; j=1}^{n} A_{i=1}^{\tilde{A}} X^{n} y_{i j}^{\alpha} y_{j} F_{f} \\
& =x^{n} @^{X^{n}} A_{i}^{\infty} l\left(y_{i}^{a} y_{j}\right) F_{F} A
\end{aligned}
$$

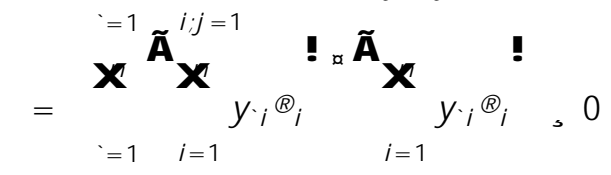

3) implica 1)

Sea $\mathrm{h}$ en subespacios cíclicos ortogonales y i $2 \mathrm{~h}$ un vector cíclico, es decir $\mathrm{fa}_{i}$ : a 2 Ug es denso en h: Ver [3] Teorema 2;1;10, pág.60.

Por hipótesis se tiene que

$$
{ }_{1 \cdot i ; j \cdot n} r_{i} i ; x_{i j} a_{j} i^{i}, 0 ;
$$

ahora sean $i_{1} ;: \ldots ; i_{n} 2 \mathrm{~h}$ entonces existe f $a_{i k} g_{k=1}^{1}$ tal que $a_{i k} i_{k !}{ }_{1} \quad i_{i}$ para $\mathrm{i}=1 ;:: ;$;: Con lo cual se tiene que

$$
h_{i} ; x_{i j} i_{j} i=\lim _{k !} h_{i k} a_{i k} ; x_{i j} a_{j k} i
$$

Por lo tanto,

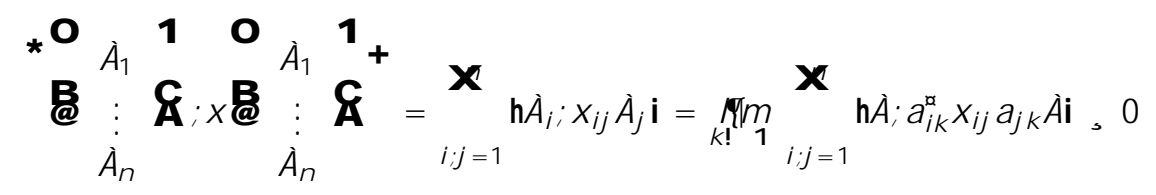

lo cual dice que $x$ es positivo.

Proposición 1.0.18 Sea T : B (h) ! B (h) una transformación lineal. Las siguientes condiciones son equivalentes:

1) $\mathrm{T}$ es $\mathrm{CP}$;

2) Para cada $n, 1$; la transformación lineal $T_{n}$ es positiva. 
Demostración. Seguiremos [8], pág.18.

1) implica 2)

Sea $x$ cualquier elemento positivo entonces por 1) y 2) de la Proposición anterior tenemos que

$$
x=x_{=11 \cdot i ; j \cdot n}^{x} y_{y_{i}^{\prime} y_{j}-E_{i j},}
$$

y como T es CP

$$
\begin{aligned}
& X^{n} X \underset{1}{x} T\left(y_{i j}^{o} y_{j j}\right)-E_{i j} \\
& =11 ; j \cdot n
\end{aligned}
$$

es positivo. Con lo cual resulta que $T_{n}(x)$ es positiva, por lo tanto $T_{n}$ es positiva.

2) implica 1)

Supongamos que para cada $n, 1 ; T_{n}$ es positiva entonces usando 2) y 3) de la proposición anterior tenemos que

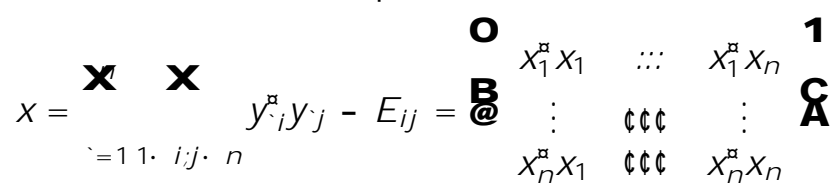

$$
\begin{aligned}
& \text { es un elemento positivo en } \boldsymbol{\beta}(b)-M_{n} ; T_{n}(x), 0 y \quad 10
\end{aligned}
$$

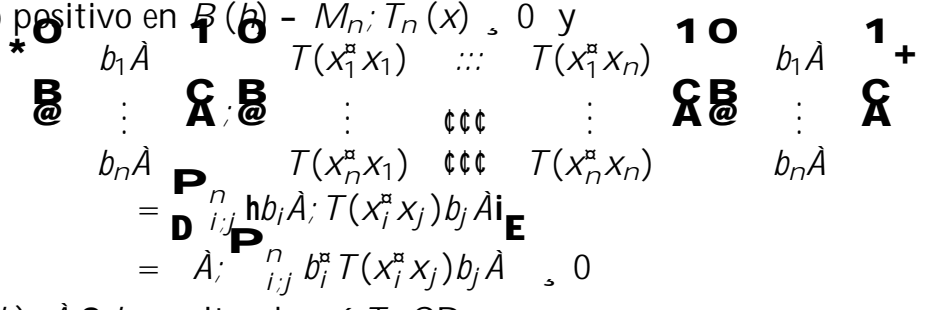

donde b 2 B (h) ; ¿ 2 h;resultando así T CP.

Proposición 1.0.19 Sea T : B (h) ! B (h) una transformación lineal: Entonces $\mathrm{T}$ es completamente positiva si y sólo si para cada $\mathrm{n}, 1$ y cada $\mathrm{u}_{1} ;:: ; \mathrm{u}_{\mathrm{n}} 2 \mathrm{~B}(\mathrm{~h}), \mathrm{k}_{1} ;:: ; ; \mathrm{k}_{\mathrm{n}} 2 \mathrm{~h}$ se tiene lo siguiente

$$
{ }_{1 \cdot i ; j \cdot n} \quad k_{i} ; T\left(u_{i}^{\alpha} u_{j}\right) k_{j} i, 0:
$$

Demostración. Seguimos [8], pág.19.

Sean $b_{1} ;: ; ; b_{n}, u_{1} ;:: ; u_{n} 2$ B (h) y $k 2$ h entonces el resultado se tiene como consecuencia de las piguientes igualdades:

$k_{i p} 1 \cdot i ; j \cdot n h^{\alpha} T\left(u_{i}^{a} u_{j}\right) b k i={ }_{1} \cdot i ; j \cdot{ }_{n} h k ; b^{\alpha} T\left(u_{i}^{\alpha} u_{j}\right) b k i$ $=1 \cdot i ; j \cdot{ }_{n} h b k ; T\left(u_{i}^{a} u_{j}\right) b k i={ }_{1} \cdot i ; j \cdot{ }_{n} h k_{i} ; T\left(u_{i}^{a} u_{j}\right) k_{j} i, 0$; donde hemos tomado la correspondencia de $b_{k}=k_{i} ; 1 \cdot i \cdot n$ : 
Proposición 1.0.20 Sea T : B (h) ! B (h) una tranformación lineal 2-positiva. Entonces se tiene lo siguiente:

1) Si T (1) es invertible en B (h) entonces para todo u 2 B (h) se tiene la siguiente desigualdad

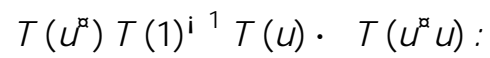

2) Para todo u 2 B (h) se tiene la desigualdad

$$
T\left(u^{\ltimes}\right) T(u) \cdot j j T(1) j_{1} T\left(u^{\alpha} u\right) \text { : }
$$

3) $T$ es continuo $y$

$$
\mathrm{jJT}_{\mathrm{j}} \mathrm{j}_{\mathrm{B}(\mathrm{B}(\mathrm{h}))}=\mathrm{jjT}(1) \mathrm{jj}_{1}:
$$

Demostración. Ver. [8]; P rop.2;10, págs.19 i 20.

\subsection{El espacio $\varnothing(\mathrm{h})$}

En esta pequeña sección $\varnothing(h)$ denota al espacio de los operadores de traza ..nita en el espacio de Hilbert h. Cada $\Omega 2 \varnothing(\mathrm{h})$ tiene la expresión

$$
\Omega={ }^{X} Æ_{n} j e_{n} i f_{n} j
$$

donde $\epsilon_{4}, 0$ con la propiedad, ${ }^{P}{ }_{n} \epsilon_{n}<1$ y fe $g_{n}, f_{n} g_{n}$ son sistemas ortonormales de $h$.

El operador je $i f_{n} j$ evaluado en $i 2 \mathrm{~h}$ tiene la expresión, je $i \mathrm{ff}_{\mathrm{n}} \mathrm{j} i=$ $h_{i} ; f_{n} i e_{n} ; y$ además je $i f_{n} j^{\infty}=j f_{n} i h_{n} j$ : Como caso particular cuando $f_{n}=e_{n}$, $\Omega$ resulta ser un operador positivo y $\Omega^{\alpha}=\Omega$ Una función sobre estos operadores que se usa para de..nir a la topología æedébil en B (h) es la función traza en $\varnothing(h)$ : Existen muchos resultados referentes a $\varnothing(\mathrm{h})$ que por el momento no se examinan aquí, pero se pueden consultar en [21] pág.7, [18] Capítulo I, dos de estos resultados útiles son: $\varnothing(h)$ es un ideal bilateral en $B(h)$; es decir dado $\Omega 2 \varnothing(\mathrm{h})$ y x 2 B (h); $\Omega$; $x \Omega 2 \varnothing(h)$ : El segundo resultado a..rma que $\operatorname{tr}(\Omega x)=\operatorname{tr}(x \Omega)$ : La relación existente entre $\varnothing(h)$ y $B(h)$ (Teorema de Schatten) [18], pág.51;es $\varnothing(h)^{\ltimes}=B(h)$ aunque no es cierto que $B(h)^{\alpha}=\varnothing(h)$; pues $\varnothing(h)$ no es rełexivo [17] págs.64-77 y 167.

De..nición 1.1.1 La función traza tr en $\varnothing(h)$ se de..ne de la siguiente forma

$$
\operatorname{tr}(\Omega)={ }_{m=1}^{X} \operatorname{hr}_{m} ; \underline{a}_{m} i ;
$$


con $\mathrm{f} \underline{a}_{m} g_{m}$ sistema ortonormal de $h$ y $\Omega 2 \varnothing(h)$ :

Proposición 1.1.2 Para cada X 2 B (h) y $\Omega, 0$ se tiene que

$$
\operatorname{tr}(\Omega \mathrm{X})={ }_{n}^{\mathrm{X}} \mathscr{F}_{\mathrm{h}} h \mathrm{X} \mathrm{e}_{\mathrm{n}} ; \mathrm{e}_{\mathrm{n}} \mathrm{i} .
$$

Demostración. Sea $f e_{n} g_{n}$ sistema ortonormal de $h$ completo,

$$
\begin{aligned}
& \operatorname{tr}(\Omega X)={ }^{X} \operatorname{rX} e_{m} ; e_{m} i=^{X{ }^{\tilde{A}} X} \rightleftharpoons \epsilon_{m} h X e_{m} ; e_{n} i e_{n} ; e_{m} i \\
& ={ }_{m}^{X^{m}} A_{m} h X e_{m} ; e_{m} i e_{m}^{m} ; e_{m}^{n} i={ }_{m}^{X} \mathscr{E}_{m} h X e_{m} ; e_{m} i:
\end{aligned}
$$

Proposición 1.31.3 Dado $\Omega 2 \varnothing(\mathrm{h})$ entonces $\mathrm{j} \Omega=\left(\Omega \Omega^{\frac{1}{2}} 2 \varnothing(\mathrm{h})\right.$ y la asociación $\Omega$ ! $\operatorname{tr}\left(\Omega \Omega^{\frac{1}{2}}\right.$ de..ne una norma en $\varnothing(h)$, el cual hace a $\varnothing(h)$ un espacio de Banach.

Demostración. Ver. [18]; págs.43 i 53.

Teorema 1.1.4 (Schatten) Sea h algún espacio de Hilbert complejo separable.

1) $\mathrm{jj}_{\mathrm{j}} \mathrm{gj}_{1}=\operatorname{tr}(\mathrm{j} \Omega$ y el espacio $\varnothing(\mathrm{h})$ es isométricamente isomorfo al dual de $\mathrm{C}(\mathrm{h})$; espacio de los operadores compactos en $\mathrm{h}$ con la norma de operadores, bajo la correspondencia $f_{\Omega}(X)=\operatorname{tr}(\Omega X)$ con $\Omega 2 \varnothing(h)$..jo y X $2 C(h)$ : 2) $B(h)$ es isométricamente isomorfo al dual de $\varnothing(h)$ con la norma jj:jj, bajo la correspondencia $f_{X}(\Omega=\operatorname{tr}(\Omega X)$ con $\Omega 2 \varnothing(h)$ y X 2 B (h) ..jo.

Demostración. Ver. [18]; pág.51.

\subsection{Topologías en $B(h)$}

Para una exposición de conceptos de topológicos como conjunto dirigido y red, el lector puede consultar [22], Capítulo I.

De..nición 1.2.1 Un espacio topológico es un par $(X ; \varnothing)$ donde $X$ es un conjunto y $\varnothing$ es una familia de subconjuntos de $X$; a para la cual se satisfacen las siguienrtes condiciones:

(1) $\odot 2 \varnothing$ y $\times 2 \varnothing ;$

(2) Si A y B $2 \varnothing$, entonces A \B $2 \varnothing$;

(3) Si U $1 / 2 \varnothing$, entonces [ U 2 ø:

Los elementos de $X$ se llaman puntos de $(X ; \varnothing)$ y los elementos de la familia $\varnothing$ se llaman subconjuntos abiertos del espacio $(X ; \varnothing)$ : R ecuérdese que el conjunto [ U es la unión de la familia U: 
De..nición 1.2.2 Sea $\pitchfork$ un conjunto no vacío. Una relación $R$ en $\pitchfork \notin \notin$ (o sea un conjunto $R \frac{1}{2} \pitchfork \notin \phi$ ) se llama dirección en $\$ ;$ si

(1) SRs, para todo $s \quad \&$;

(2) $S R t$ y tR $i$ implica $S R_{i}$, para todo $s ; t ; i 2 \phi$;

(3) Para todo s; t $2 \notin$ existe un $i 2 \notin$ tal que sR $i$ y sRt:

Si R es una dirección en $\$$, entonces $\$$ se llama dirigido por $R$.

De..nición 1.2.3 Sea $X$ un espacio topológico. Una red $S$ en $X$ es cualquier mapeo de un conjunto dirigido(que se llama dominio) en $X$.

Si el conjunto dirigido es de los número naturales con el orden usual, entonces $\mathrm{S}$ se llama sucesión. E $n$ muchas ocaciones una red en $\mathrm{X}$ se denota por $\left(\mathrm{X}_{\mathrm{t}}\right)_{\mathrm{t} 2 \mathrm{q}}$ donde $\phi$ es el dominio de $S$.

De..nición 1.2.4 Dado un espacio toposógico $X$ y un punto $x_{0} 2 X$, diremos que una red $\mathrm{S}=\left(\mathrm{X}_{\mathrm{t}}\right)_{\mathrm{t} 2 \Phi}$ converge a $\mathrm{x}_{0} \mathrm{~S} !_{\mathrm{t}} \mathrm{x}_{0}$ si para toda vecindad $\mathrm{U}$ de $\mathrm{x}_{0}$ en $X$ existe $t_{0} 2 \notin$ tal que $X_{t} 2 U$ para todo $t, t_{0}$ :

De..nición 1.2.5 La topología fuerte en $B(h)$, es la topología inducida por la familia de seminormas $\mathrm{fP}_{i}: i 2$ hg de..nidas por $\mathrm{P}_{i}(X)=j \mathrm{j} X(i) \mathrm{jj} \mathrm{h}$ :

De..nición 1.2.6 La topología débil en $B(h)$; es la topología inducida por la

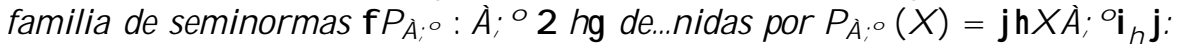

De..nición 1.2.7 La topología ultradébil (ædébil ) en B (h) ; es la topología inducida por la familia de seminormas $\mathrm{fP}_{\Omega}: \Omega 2 \varnothing(\mathrm{h}) \mathrm{g}$ de..nidas por

$$
P_{\Omega}(X)=j \operatorname{tr}(\Omega X) j:
$$

Cabe mencionar que $P_{\Omega}(X)$ está bién de. nida porque como ya mencionamos antes $\varnothing(h)$ es un ideal bilateral en $B(h)$. 
Proposición 1.2.8 Sea $₫$ un conjunto dirigido. Una red $f X_{i} g_{i 2 屯}$ en $B(h)$ converge a $X 2$ B (h):

1)Fuertemente si y sólo si $j \mathrm{j} \mathrm{X}_{\mathrm{i}}\left(\int\right) \mathrm{i} X\left(\int\right) \mathrm{jj}_{\mathrm{h}}$ ! 0 ; para todo $\int 2 \mathrm{~h}$ :

2) Débilmente si y sólo si $j h \mathrm{X}_{i} i_{i} \int \mathrm{i}_{\mathrm{h}} \mathrm{i} h \mathrm{X}_{i ;} ; \mathrm{i}_{\mathrm{h}} \mathrm{j}$ ! 0 ; para todo $\int ; i 2 \mathrm{~h}$ :

3) Ultradébilmente si y sólo si jtr $\left(\Omega\left(X_{i}\right.\right.$ i $\left.\left.X\right)\right)$ j ! 0 ; para todo $\Omega 2 \mathrm{~h}$ :

Demostración. Es inmediata de las de..niciones 1.2.1, 1.2.2 y 1.2.3.

Proposición 1.2.9 La red $f X_{i} g_{i 2 屯}$ converge ultradébilmente $a_{p} X 2 \mathrm{~B}(\mathrm{~h})$ si y sópo si para cualesquiera $f \int n g ; f_{i n} g$ sucesiones en $h$ tales que ${ }_{n} j j \int n j j h$ y ${ }_{n} \mathrm{jj}_{i n} \mathrm{nj}_{\mathrm{h}}^{2}<1$ se tiene que

$$
{ }_{n} \not X_{i} \int_{n} i_{i n} i_{h} !_{i}{ }_{n}^{X} h \int_{n} ; i_{n} i_{h}:
$$

Demostración. Tomando $\Omega={ }^{P}{ }_{n} / F_{n} j e_{n} i e_{n} j$ y $\int_{n}=i_{n}=A^{\frac{1}{2}} e_{n}$ se obtiene el resultado.

Lema 1.2.10 ( Dini) Sea $\pitchfork$ un conjunto dirigido. Sean $f_{\mathbb{E} ;} f: K$ ! $R$ con-

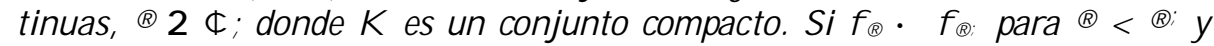

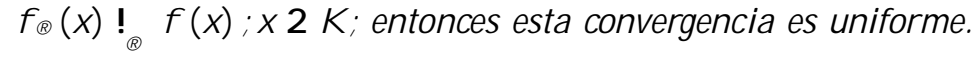

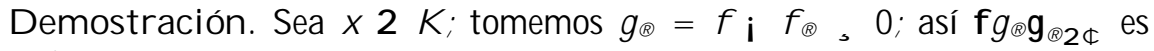
decreciente $y$

I $_{\mathbb{E}} g_{\mathscr{E}}(\mathrm{x})=0$ : Por lo tanto dado " $>0$ existe una vecindad $\mathrm{U}$ de $\mathrm{x}$

y algún $\mathscr{E}_{x}$ tal que $g_{\mathbb{E}}(\mathrm{x})<$ "; para $\mathscr{E}_{E}, \mathscr{F}_{x}$ : A hora para este " >0;

considér ese $\mathrm{fU}_{\mathrm{x}}: \times 2 \mathrm{Kg}$ una cubierta de $\mathrm{K}$, por la compacidad

de $\mathrm{K}$ existe un $\mathrm{n} 2 \mathrm{~N}$ y $\mathrm{x}_{1} ;: ;: ; \mathrm{x}_{\mathrm{n}} 2 \mathrm{~K}$ tal que $\mathrm{K}=\left[\mathrm{i}=1 \mathrm{U} \mathrm{U}_{\mathrm{i}}\right.$; sea

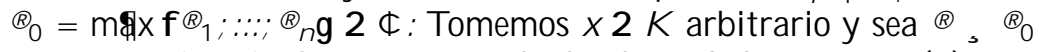
entonces existe $i$ tal que $x 2 U_{x_{i}}$ de donde se deduce que $g_{k}(x)<"$; lo cual quiere decir que jf $f_{\mathbb{E}}(x)$ i $f(x) j<"$ "; el cual es la propia convergencia uniforme.

Teorema 1.2.11 La topología ultradébil y la débil coinciden en subconjuntos acotados de $B(h)$ :

Demostración. Seguimos [8], pág.9.

Sea $B(0 ; 1)=f \times 2 B(h): \mathrm{jjxjj}_{1} \cdot 1 \mathrm{~g}$, como $\varnothing(h)^{\ltimes}=B(h)$ entonces resulta que $\left(B(0 ; 1) ; \varnothing_{\not a}\right.$ dibil $)$ es compacto por el Teorema de A laoglu y por el teorema del mapeo abierto basta tomar

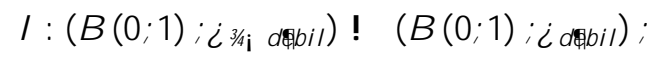

claramente I la función identidad, es una biyección continua y como las topologías son de Hausdora, se tiene que ambas topologías coinciden. 
De..nición 1.2.12 Un álgebra de von Neumann U es una C*-subálgebra de $B(h)$ con identidad la cual es cerrada en la topología débil.

Ejemplo 1.2.13 Toda álgebra de von-Neumann es una $C^{\circledR}$-álgebra, pero no inversamente. Para ello tómese el siguiente ejemplo.

Sea $C^{0}=f f:[0 ; 1]$ ! $\quad C: f$ es continuag ${ }^{1 / 2} B(h) ;$ donde $h=L^{2}([0 ; 1] ; \pi)$ siendo $\pi$ la medida de Lebesgue: Ahora con $\mathrm{jjf}_{\mathrm{j} j} \mathrm{j}_{1}=\operatorname{supj} \mathrm{f}(\mathrm{x}) \mathrm{j}$ y $\mathrm{f}^{\mathrm{x}}(\mathrm{x})=$ $\overline{f(x)}$ se tiene que $C^{0}$ es una $C^{\alpha}$-subálgebra de $B(h)$. A hora sea $\left.\neg 0 ; \frac{1}{2}\right]$; claramente se tiene que $\left.\neg 0 ; \frac{1}{2}\right]=C^{0}$ : Pero existe $\mathrm{ff}_{\mathrm{n}} \mathrm{g}^{1 / 2} \mathrm{C}^{0}$ tal que $\left.\left.\mathrm{f}_{\mathrm{n}}{ }_{\mathrm{n}} !{ }_{1}\right] 0 ; \frac{1}{2}\right]$ puntualmente, donde

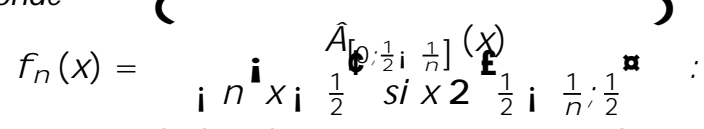

Para ver que $\mathrm{C}^{0}$ no es un álgebra de von Neumann, veámoslo contenido en $B(h)$ mediante el mapeo que asocia $f 2 C^{0} ; f$ ! $m(f) 2 B(h)$ sienso $m(f)$ el operador de multiplicaçión por, $f$ : De aquí se sigue que $\left.m\left(f_{n}\right) ! s m \neg 0 ; \frac{1}{2}\right]$ pero esto implica que $\left.\mathrm{m} \backslash 0 ; \frac{1}{2}\right]$ está en $\mathrm{m}^{\mathrm{i}} \mathrm{C}^{0^{\phi}}$ cosa que no puede ser ya que $\left.\neg 0 ; \frac{1}{2}\right] \mathrm{C}^{0}$;resultando así que $\mathrm{C}^{0}$ no es álgebra de von Neumann.

P roposición 1.2.14 Sea U un álgebra de von Neumann de operadores actuando sobre un espacio de Hilbert $h$. Sea 4 un conjunto dirigido y $f X_{i} g_{i 2 \downarrow}$ una red creciente de elementos de $U$ tales que Supjj $X_{i} j_{1}<1$ : Entonces existe $X 2$ $U$ tal que $X_{i} ! X$ en la topología débil, ultradébil y fuerte. $A X$ se le denota también con $X \underset{i 2 \Phi}{=} \operatorname{SupX}_{i}$.

Demostración. Ver. [3] Teorema 2;4;21; pág: 76:

De..nición 1.2.15 Sea U un álgebra de von Neumann $\mathrm{y}^{\prime}$ ' fungional lineal positiva en U: Se dice que ' es normal si $\operatorname{Sup}_{i 2 \Phi}\left(X_{i}\right)={ }^{\prime} \underset{i 2 \Phi}{\operatorname{SupX}_{i}}$ :

Teorema 1.2.16 Sea $T: B(h)$ ! B (h) lineal, positivo y $æ$ débil continuo entonces existe $S: \varnothing(h) ! \varnothing(h)$ tal que

$$
\operatorname{tr}(S(\Omega)=\operatorname{tr}(\Omega T(x))
$$

para todo $\Omega 2 \varnothing(\mathrm{h})$ y $2 \times 2 \mathrm{~B}(\mathrm{~h})$ :

Demostración. Ver. [3] Teorema 2;4;20; págs.76 ; 78: 
Proposición 1.2.17 a) Sean T ; E : B (h) ! B (h) transformaciones lineales completamentes positivas entonces $\mathrm{E}+\mathrm{T}$ es completamente positiva.

b) $E \pm T$ : B (h) ! B (h) es completamente positiva.

c) Sea $\mathrm{K}$ un espacio de Hilbert y $\mathrm{fT}_{\mathrm{m}} \mathrm{g}_{\mathrm{m}}$ una sucesión transformaciones completamentes positivas, $\mathrm{T}_{\mathrm{m}}: \mathrm{B}(\mathrm{h})$ ! $\mathrm{B}(\mathrm{K})$ : Supóngase que para cada u 2 $B(h)$ la sucesión $\mathrm{fT}_{\mathrm{m}}(\mathrm{u}) \mathrm{g}_{\mathrm{m}},{ }_{1}$ converge débilmente. Entonces la transformación lineal $T: B(h)$ ! $B(K)$ de..nida por $T(u)=\lim _{m} T_{m}(u)$ es completamente positiva.

D emostración. Seguimos [8], págs.20-21.

a) Sean $b_{1} ;: ; ; b_{n}$ y $u_{1} ;: ; ; u_{n} 2 \mathrm{~B}(h)$ entonces

$$
\begin{gathered}
X \quad b^{\alpha}[E+T]\left(u_{i}^{\alpha} u_{j}\right) b \\
={ }_{1 \cdot i ; j \cdot n}^{X} b^{\alpha} E\left(u_{i}^{\alpha} u_{j}\right) b+{ }_{1 \cdot i ; j \cdot n} b^{\alpha} T\left(u_{i}^{\alpha} u_{j}\right) b, 0:
\end{gathered}
$$

b) Para esto únicamente veremos que $(E \pm T)_{n}=E_{n} \pm T_{n}$, donde $(E \pm T)_{n}: B(h)-M_{n}$ ! $B(h)-M_{n}$.

Sea $u-E_{i j} 2 B(h)-M_{n}$ entonces se tiene lo siguiente:

$$
\begin{aligned}
(E \pm T)_{n}\left(u-E_{i j}\right)=(E \pm T)(u)-E_{i j}=E(T(u))-E_{i j} \\
=E_{n}\left(T(u)-E_{i j}\right)=E_{n}\left(T_{n}\left(u-E_{i j}\right)\right)=E_{n} \pm T_{n}\left(u-E_{i j}\right)
\end{aligned}
$$

con lo cual resulta que $(E \pm T)_{n}=E_{n} \pm T_{n}$ :

c) Sea $n_{p} 1, u_{1} ; \ldots ; u_{n} 2 B(h)$ y $k_{1} ; \ldots ; k_{n} p^{2} K$

$$
1 \cdot i ; j \cdot{ }_{n} k_{i} ; T\left(u_{i}^{\alpha} u_{j}\right) k_{j} i=\lim _{m}\left[m_{1} 1 \cdot i ; j \cdot{ }_{n} k_{i} ; T_{m}\left(u_{i}^{\alpha} u_{j}\right) k_{j} i, 0:\right.
$$

Teorema 1.2.18 (Stinespring) Sea h un espacio de Hilbert. ' : B (h) ! B (h) es lineal y completamente positivo si y sólo si existe $\mathrm{K}$ espacio de Hilbert y $\mathrm{L}$ $: \mathrm{h}$ ! $\mathrm{h}-\mathrm{K}$ lineal tal que

$$
\text { ' }(x)=L^{x}(x-1) L:
$$

Nota 1.2.19 El operador $x-1: h-K ! h-K$, que se de.ne como $(x-1)\left(\int-i\right)=x\left(\int\right)-i$, se llama la ampliación de $x$ en $h-K$ :

Para hacer la demostración de este teorema haremos uso de 4 resultados cuyas demostraciones se pueden encontrar en [18]; pág.251 i 254.

1) Proposición 29.2.Sea T : B $\left(h_{2}\right)$ ! B $\left(h_{1}\right)$ completamente positivo, defínase

$$
K\left(x_{1} ; y_{1} ; u_{1} ; x_{2} ; y_{2} ; u_{2}\right)=h u_{1} ; y_{1}^{a} T\left(x_{1}^{a x} x_{2}\right) y_{2} u_{2} i
$$


donde $\left(x_{i} ; y_{i} ; u_{i}\right) 2 B\left(h_{2}\right) £ B\left(h_{1}\right) £ h_{1}$ para $i=1 ; 2$ : Entonces $K$ es de..nido positivo en $B\left(h_{2}\right) \notin B\left(h_{1}\right) \notin h_{1}$; es decir dado $n 2 N$; para todo $\left(x_{i} ; y_{i} ; u_{i}\right) 2 B\left(h_{2}\right) £ B\left(h_{1}\right) £ h_{1} ; i=1 ;: . ; n$ y $\Pi_{h} ;:: ; \Gamma_{h} 2$ C;

$$
\mathrm{x}_{\mathrm{i} ; \mathrm{j}=1}^{\mathrm{n}} \Pi_{\mathrm{N}} \mathrm{K}\left(\mathrm{x}_{\mathrm{i}} ; \mathrm{y}_{\mathrm{i}} ; \mathrm{u}_{\mathrm{i}} ; \mathrm{x}_{\mathrm{j}} ; \mathrm{y}_{\mathrm{j}} ; \mathrm{u}_{\mathrm{j}}\right), 0 \text { : }
$$

2) Proposición 29.3. Sea $T: B\left(h_{2}\right)$ ! $B\left(h_{1}\right)$ completamente positivo entonces existe un espacio de Hilbert $h$ y $П: B\left(h_{2}\right) f h_{1}$ ! $h$ tal que se tiene lo siguiente:

i) $f \Pi(x ; u) j x 2$ B $\left(h_{2}\right)$; u $2 h_{1} g$ es total en $h$ :

ii) $\Pi$ es lineal en cada variable $x, u$ :

iii) $h \prod\left(x_{1} ; u_{1}\right) ; \Pi\left(x_{2} ; u_{2}\right) i=h u_{1} ; T\left(x_{1}^{a} x_{2}\right) u_{2} i$ :

iv) En particular $\int_{0}(u)=\Pi(1 ; u)$ es una isometría de $h_{1}$ ! $h$ :

3) Proposición 29.4. A partir de 2) para $\mathrm{T}$; $\Pi$ y $\int_{0}$ existe un *- homomor..smo unital $\cong$ : $B\left(h_{2}\right)$ ! $B(h) ;(\circ(1)=1)$ tal que

$$
\Pi(\mathrm{x} ; \mathrm{u})=\underline{\mathrm{o}}(\mathrm{x}) \int_{0} \mathrm{u} ; \mathrm{x} 2 \mathrm{~B}\left(\mathrm{~h}_{2}\right) ; \mathrm{u} 2 \mathrm{~h}_{1}:
$$

4) Proposición 29.5. A hora para el o construido en 3) existe un espacio de Hilbert $\mathrm{K}$ y un isomor..smo unitario $\varnothing: \mathrm{h} ! \mathrm{h}_{2}-\mathrm{K}$ tal que $o(x)=\varnothing^{1} x-1 \varnothing$ con $x 2 B\left(h_{2}\right)$ y 1 es el operador identidad en $K$ :

Ahora si ya podemos demostrar el Teorema 2;1;16.

Demostración. Demostremos la condición su..ciente. Siendo i es completamente positivo

de $B(h)$ a $B(h)$ mismo y usando los 4 resultados anteriores todos con

$\mathrm{h}=\mathrm{h}_{1}=\mathrm{h}_{2}$ y $\mathrm{L}=\varnothing \int 0 ; \mathrm{T}={ }^{\prime}$ se tiene que para $\mathrm{u}_{1} ; \mathrm{u}_{2} 2 \mathrm{~h}$

$$
\begin{aligned}
& h u_{1} ; L^{\infty}(x-1) L u_{2} i={ }^{-} u_{1} ; \int_{0}^{\infty} \varnothing^{i}{ }^{1}(x-1) \varnothing \int_{0} u_{2}{ }^{\circledR} \\
& =\mathrm{h} \int \mathrm{u}_{1} ; \underline{\mathrm{o}}(\mathrm{x}) \int_{0} \mathrm{u}_{2} \mathrm{i} \\
& =\mathrm{r}(1) \int_{0} \mathrm{u}_{1} ; \underline{\mathrm{o}}(\mathrm{x}) \int_{0} \mathrm{u}_{2} \mathrm{i} \\
& =\eta \Pi\left(1 ; u_{1}\right) ; \Pi\left(x ; u_{2}\right) \mathrm{i} \\
& =h u_{1} ; i\left(1^{x} x\right) u_{2} i \\
& =h u_{1} ; i(x) u_{2} i \text {; }
\end{aligned}
$$


por lo tanto,

$$
i(x)=L^{x}(x-1) L:
$$

A hora veamos la necesidad.

$i(x)=L^{x}(x-1) L:$

La linealidad es fácil, sean $x$ y y 2 B (h) entonces

$$
\begin{aligned}
i(x+y) & =L^{x}((x+y)-1) L \\
& =L^{x}((x-1)+(y-1)) L \\
& =L^{x}(x-1) L+L^{x}(y-1) L \\
& =i(x)+{ }^{\prime}(y):
\end{aligned}
$$

i es completamente positivo. Sea $n, 1$ y $x_{1} ;:: ; x_{n} ; y_{1} ;: ; ; y_{n} 2$ B (h).

Tomemos u $2 \mathrm{~h}$; entonces tenemos lo siguiente

$$
\begin{aligned}
& * \\
& u_{i ; j=1}^{x} y_{i}^{a} i\left(x_{i}^{\alpha} x_{j}\right) y_{j} u=x_{i ; j=1}^{x} h y_{i} u ; i\left(x_{i}^{\alpha} x_{j}\right) y_{j} u i \\
& =x_{i ; j=1}^{n} h y_{i} u ; L^{x}\left(\left(x_{i}^{\alpha} x_{j}\right)-1\right) L y_{j} u i \\
& =X_{i ; j=1}^{X}-L y_{i} u ;\left(x_{i}-1\right)^{\infty}\left(x_{j}-1\right) L y_{j} u^{\circledR} \\
& =X^{h} h\left(x_{i}-1\right) L y_{i} u ;\left(x_{j}-1\right) L y_{j} u i \\
& =x_{i=1}^{x_{*}^{i j j}=1}\left(x_{i}-1\right) L y_{i} u_{j=1}^{X^{n}}\left(x_{j}-1\right) L y_{j} u+ \\
& =j j_{j=1}^{X}\left(x_{j}-1\right) L y_{j} u j j^{2}, 0:
\end{aligned}
$$

i es ædébil continuo.

Sea $X_{F E}-1 \underset{f E}{\text { qw }} x-1$, es decir dado $\Omega 2 \varnothing(h), \operatorname{tr}\left(\Omega\left(x_{\mathbb{E}}-1\right)\right) ! \operatorname{tr}(\Omega(x-1))$

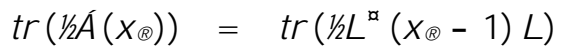

$$
\begin{aligned}
& =\operatorname{tr}\left(L \Omega^{x}\left(x_{E E}-1\right)\right) \\
& \lim _{F E}\left(\Omega_{A}\left(x_{F E}\right)\right)=\lim _{F E} \operatorname{tr}\left(L \Omega^{\infty}\left(X_{F E}-1\right)\right)=\operatorname{tr}\left(L \Omega^{\infty}(X-1)\right) \\
& =\operatorname{tr}\left(\Omega^{x}(x-1) L\right)=\operatorname{tr}\left(\Omega_{4}(x)\right)
\end{aligned}
$$

por lo tanto i es ædébil continuo. 
Teorema 1.2.20 (Krauss) La transformación lineal T : B (h) ! B (h) es normal y completamente positiva si y sólo si puede ser expresada en la forma

$$
\mathrm{T}(\mathrm{a})={ }_{\mathrm{j}=1}^{\mathrm{X}} \mathrm{V}_{\mathrm{i}}^{\mathrm{x}} \mathrm{a} \mathrm{V}_{j}
$$

Donde $f V_{j} g_{j=1}^{1}$ son operadores acotados de $h$ en $h$ mismo y la serie ${ }_{j=1}^{P} V_{i}^{\alpha} V_{j}$ converge debilmente.

Demostración. Ver. [8]; pág. 24, [13], págs. 311 i 335: 


\section{Capítulo 2}

\section{Semigrupos dinámicos cuánticos(S.D.C.)}

En este capítulo se proporciona la forma explícita que debe tener el generador in..nitesimal de un semigrupo dinámico cuántico uniformemente continuo. Los dos resultados importantes que garantizan esto y con los cuales se ..naliza este capítulo son: el Teorema de Linblad 2;1;17 y el Teorema de Linblad-GoriniK ossakowski-Sudarshan 2;1;18: 


\section{1.}

De..nición 2.1.1 Un Semigrupo Dinámico Cuántico(S. D. C) en B (B (h)) es una familia $T=\mathrm{fT}_{\mathrm{t}} \mathrm{g}_{\mathrm{t}}, 0$ de operadores acotados con las siguientes propiedades:

1) $T_{0}(x)=x, \quad x 2 B(h)$;

2) $T_{t+s}(x)=T_{t}\left(T_{s}(x)\right)$, para s; $t, 0 ; x 2 B(h)$;

3) $T_{t}$ es completamente positivo para todo $t, 0$;

4) $T_{t}$ es æedébil continuo en $B(h)$ para todo $t, 0$..jo;

5) Para cada $x 2 \mathrm{~B}(\mathrm{~h})$..jo, el mapeo $t$ ! $T_{t}(x)$ es continuo respecto a la topología ædébil en $B(h)$ : [8], pág.28.

De..nición 2.1.2 El generador in..nitesimal de un Semigrupo Dinámico Cuántico T. Es el operador $\$$, cuyo dominio $D(\$)$ es el espacio de los elementos de $B(h)$ para los cuales existe un elemento b2 B (h) tal que

$$
\mathrm{b}=\lim _{\mathrm{t} !} \min _{0} \frac{\mathrm{T}_{\mathrm{t}}(\mathrm{x}) \mathrm{i} \mathrm{x}}{\mathrm{t}}
$$

en la topología ædébil, y para a $2 \mathrm{D}(\$) ; \$(\mathrm{a})=\mathrm{b}$

De..nición 2.1.3 Un semigrupo $T=\mathrm{fT}_{\mathrm{t}} \mathrm{g}_{\mathrm{t}, 0}$ en $\mathrm{B}(\mathrm{h})$, es decir $\mathrm{T}$ satisface 1) y 2) de la De.nición 2;1;1: Es de contracciones si $\mathrm{jj}_{\mathrm{t}} \mathrm{tj}_{\mathrm{B}(\mathrm{B}(\mathrm{h}))} \cdot 1$; donde $j j T_{t} j_{B}(B(h))=\operatorname{Sup}_{\substack{x 2 B(h) \\ j j x j j=1}} j j T(x) j j_{B}(h):$

N ota 2.1.4 T es llamado uniformemente continuo si

$$
\lim _{\mathrm{t} !} \mathrm{m}_{0} k \mathrm{~T}_{\mathrm{t}} \mathrm{i} \mathrm{T}_{0} \mathrm{k}_{\mathrm{B}(\mathrm{B}(\mathrm{h}))}=0 \text { : }
$$

Proposición 2.1.5 Sea $(-; z ; \pi)$ un espacio medible con $\pi$ medida ...nita sobre $z$, y $f U(t ; x) g_{t, 0 ; x z \text {. }}$ una familia de operadores acotados sobre un espacio de

Hilbert $h$ tal que:

1) Para todo $x 2$ - ; la transformación $t$ ! $U(t ; x)$ es fuertemente continua, es decir para cada i $2 \mathrm{~h}$; $\mathrm{t}$ ! $\mathrm{U}(\mathrm{t} ; \mathrm{x})$ i es continua;

2) Para todo $t, 0$; la transformación $x$ ! $U(t ; x)$ es fuertemente medible, es decir dado dado $i 2 \mathrm{~h} ; \mathrm{x} ! \mathrm{U}(\mathrm{t} ; \mathrm{x}) i$ es medible;([17] Teorema IV.22, págs.116 i 117).

3) Para todo $t, 0$, existe una función positiva g sobre - integrable con respecto a $\pi$; tal que

$$
\operatorname{Sup}_{0 . \mathrm{s} \cdot \mathrm{t}} \mathrm{kU}(\mathrm{s} ; \mathrm{x}) \mathrm{k}^{2} \cdot g(\mathrm{x}) ; \quad \operatorname{con} g \mathrm{t} 2 \mathrm{~L}^{1}(-; z ; \pi) \text { : }
$$

Entonces la transformación a : [0; 1 ) f B (h) ! B (h) de..nida por la inte- 
gral

$$
\mathrm{a}(\mathrm{t} ; \mathrm{a})=\mathrm{-}_{-}^{\mathrm{Z}} \mathrm{U}(\mathrm{t} ; \mathrm{x})^{\mathrm{a}} \mathrm{aU}(\mathrm{t} ; \mathrm{x}) \mathrm{dr}(\mathrm{x})
$$

es ædébil continua en ambos argumentos y completamente positiva en la segunda variable.

Demostración.

Seguimos [8], págs.32-33.

Observemos que

$0 \cdot \mathrm{hU}(\mathrm{t} ; \mathrm{x}) \int ; \mathrm{aU}(\mathrm{t} ; \mathrm{x}) \int \mathrm{i} \cdot \mathrm{kU}(\mathrm{t} ; \mathrm{x}) \int \mathrm{kkaU}(\mathrm{t} ; \mathrm{x}) \int \mathrm{k} \cdot \mathrm{kak}_{1} \mathrm{kU}(\mathrm{t} ; \mathrm{x}) \int \mathrm{k}^{2}$

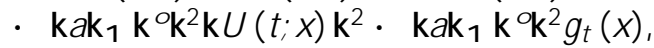

así como función de $\mathrm{x}$ resulta integrable, y por lo tanto

$Z$

$\mathrm{hU}(\mathrm{t} ; \mathrm{x}) \int ; \mathrm{aU}(\mathrm{t} ; \mathrm{x}) \int \mathrm{i} d \pi(\mathrm{x})$

de. ne una forma sesquilineal acotada que está representada por un operador al que denotaremos por $\underline{a}(\mathrm{t} ; \mathrm{a})$ o por $\mathrm{U}(\mathrm{t} ; \mathrm{x})^{\mathrm{a}} \mathrm{aU}(\mathrm{t} ; \mathrm{x}) \mathrm{dr}(\mathrm{x})$. Veamos que a es completamente positivo en la segunda variable, para eso tomando $t . . j o$, sea $n, 1 ; a_{1} ; \ldots ; a_{n} ; b_{1} ; \ldots ; b_{n} 2$ B (h)

$$
\begin{aligned}
& \int_{i ; j=1}^{x^{n}} b^{\alpha a}\left(t ; a_{i}^{\alpha} a_{j}\right) b \int_{i ; j=1}^{+}=x^{n} \operatorname{lb} \int ; \underline{a}\left(t ; a_{i}^{\alpha} a_{j}\right) b \int i
\end{aligned}
$$

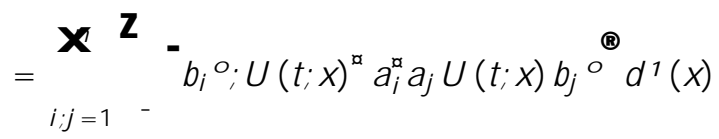

$$
\begin{aligned}
& =x_{i ; j=1}^{x^{n} Z} r_{i} U(t ; x) b \int ; a_{j} U(t ; x) b \int i d \pi(x) \\
& =Z x^{n} r a_{i} U(t ; x) b \int ; a_{j} U(t ; x) b \int i d \pi(x) \\
& z^{i ; j=1} \\
& =k_{i=1}^{X^{n}} a_{i} U(t ; x) b \int k^{2} d \pi(x), 0
\end{aligned}
$$

por lo tanto tenemos que a es completamente positivo, en la segunda variable operador.

Enseguida demostramos que es ædébil continuo en el tiempo. Para ello tomemos s; t $2[0 ; r]$ :

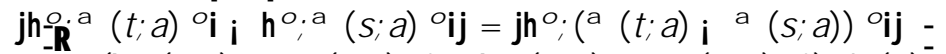

$=\mathrm{R}-\left(\mathrm{hU}(\mathrm{t} ; \mathrm{x}) \int ; \mathrm{aU}(\mathrm{t} ; \mathrm{x}) \int \mathrm{i} \mathrm{i} \mathrm{hU}(\mathrm{s} ; \mathrm{x}) \int ; \mathrm{aU}(\mathrm{s} ; \mathrm{x}) \int \mathrm{i}\right) \mathrm{dr}(\mathrm{x})$

- R jhU $(t ; x) \int ; a(U(t ; x)$ i $U(s ; x)) \int i j d \pi(x)+$

. $j h(U(t ; x)$ i $U(s ; x)) \int ; a U(s ; x) \int i j d r(x)$ 
el Rrimer sumando de esta desigualdad lo estimamos por

- $\mathrm{kU}_{3}(\mathrm{t} ; \mathrm{x}) \int k \mathrm{kak}_{1} \mathrm{k}\left(\mathrm{U}(\mathrm{t} ; \mathrm{x}), \mathrm{i} \mathrm{U}_{3}(\mathrm{~s} ; \mathrm{x})\right) \int \mathrm{kdm}(\mathrm{x})$

- $\mathrm{kak}_{1}{ }^{\mathrm{R}} \mathrm{kU}(\mathrm{t} ; \mathrm{x}) \int \mathrm{k}^{2} \mathrm{dr}(\mathrm{x})^{\frac{1}{2}^{3} \mathrm{R}} \mathrm{-}(\mathrm{U}(\mathrm{t} ; \mathrm{x}) ; \mathrm{U}(\mathrm{s} ; \mathrm{x})) \int \mathrm{k}^{2} \mathrm{dr}(\mathrm{x})^{\frac{1}{2}}$

- $k a k_{1}{ }^{i R}-g_{r}(x) d \pi(x)^{\phi_{\frac{1}{2}}{ }^{3} R}-k(U(t ; x) i U(s ; x)) \int k^{2} d \pi(x)^{\frac{1}{2}}$

pero de aquí mismo obsérvese que

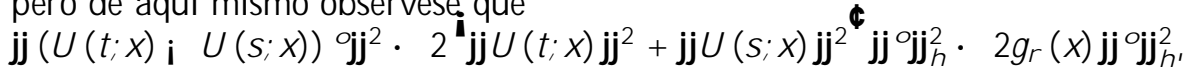
entonces por el Teorema de Convergencia Dominada de Lebesgue, se concluye que el primer sumando tiende a 0 ; conforme $t$ ! s; un análisis similar muestra que el segundo sumando también tiende a 0 conforme $t$ ! s:

Enseguida demostramos que a es æedébil continuo en el segundo parámetro. B astará ver que a $\left(\mathrm{t}\right.$; a) es normal, es decir que ha (t; a) $\int ; \int i=\operatorname{tr}(\Omega \mathrm{a})$ donde $\Omega$ es un operador de traza ...nita en $\varnothing(h)$ y $\int 2 h$, para ello tomemos

$$
\Omega={ }^{Z} j U(t ; x) \int i h U(t ; x) \int j d \pi(x)
$$

y comprobemos que en verdad este $\Omega$ es el adecuado, sea $f$ e $g_{i=1}^{1}$ una base ortonormal para nuestro h: Vamos a demostrar que

$$
X_{i=1}^{A} \text { he; } \text { నq }_{i}<1 \text { : }
$$

Es claro que

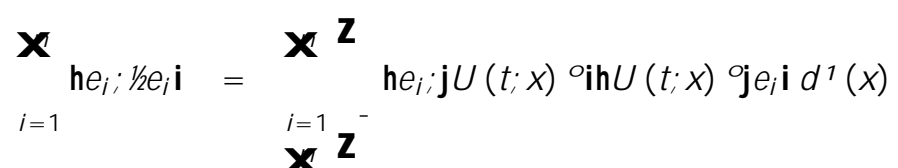

$$
\begin{aligned}
& =h_{e} ; \mathrm{hU}(\mathrm{t} ; \mathrm{x}) \int ; \mathrm{e} i \mathrm{U}(\mathrm{t} ; \mathrm{x}) \int \mathrm{i} d \pi(\mathrm{x}) \\
& =x_{i=1}^{i=1} z^{-} j h U(t ; x) \int ; e i j^{2} d \pi(x) \\
& ={ }_{-i=1}^{Z} j h U(t ; x) \int ; e i j^{2} d \pi(x) \underset{n ! 1}{!_{1}}{ }_{-i=1}^{Z} j h U(t ; x) \int ; e_{i j} j^{2} d \pi(x)
\end{aligned}
$$

y usando la fórmula de Parseval se tiene que

$$
\begin{aligned}
& { }_{i=1}^{X} h_{1} ; \Omega_{1} i={ }_{-i=\frac{Z}{Z}}^{Z} j h U(t ; x) \int ; e_{i} i j^{2} d \pi(x)={ }_{-Z}^{Z} j j U(t ; x) \int j j^{2} d \pi(x) \\
& \text { - } \quad j j \int j j_{h}^{2} \quad j j U(t ; x) j j^{2} d \pi(x) \cdot j j \int j j_{h}^{2} \quad g_{r}(x) d \pi(x)<1 \text {; }
\end{aligned}
$$

así $\Omega 2 \varnothing(\mathrm{h})$ : 
Pero por otra parte recordemos que: $\mathrm{R}$

$\mathrm{h} \int ; \underline{\mathrm{a}}(\mathrm{t} ; \mathrm{a}) \int \mathrm{i}=\operatorname{tr}\left(\mathrm{j} \int \mathrm{ih}(\mathrm{t} ; \mathrm{a}) \int \mathrm{j}\right)=\mathrm{hU}(\mathrm{t} ; \mathrm{x}) \int ; \mathrm{aU}(\mathrm{t} ; \mathrm{x}) \int \mathrm{i} d \pi(\mathrm{x})$

por lo tanto

$$
\begin{aligned}
& \operatorname{tr}(\Omega \mathrm{a})=\mathrm{x}_{\mathrm{i}=1}^{\mathrm{Z}} \mathrm{he}_{1} ; \mathrm{HU}(\mathrm{t} ; \mathrm{x}) \int ; \operatorname{ae} i \mathrm{U}(\mathrm{t} ; \mathrm{x}) \int \mathrm{i} d \pi(\mathrm{x}) \\
& X^{i=1} Z \\
& =\quad h U(t ; x) \int ; a e i h_{i} ; U(t ; x) \int i d r(x) \\
& i=1 Z^{-} \\
& =\quad \operatorname{ra}^{\mathrm{x}} \mathrm{U}(\mathrm{t} ; \mathrm{x}) \int \text {; ei he; } \mathrm{U}(\mathrm{t} ; \mathrm{x}) \int \mathrm{i} d \pi(\mathrm{x}) \\
& x^{i=1} Z^{-} \\
& =\quad h U(t ; x) \int ; a U(t ; x) \int i d \pi(x) \\
& i=1 \text {. } \\
& =\mathrm{h} f ; \underline{\underline{a}}(\mathrm{t} ; \mathrm{a}) \int \mathrm{i} \text { : }
\end{aligned}
$$

Así hf ; a (t; a) $\int \mathrm{i}=\operatorname{tr}(\Omega \mathrm{a})$; lo que prueba que a es normal.

De..nición 2.1.6 Un Semigrupo Dinámico Cuántico $\mathrm{fT}_{\mathrm{t}} \mathrm{g}_{\mathrm{t}, 0}$ es llamado conservativo si $T_{t}(1)=18 \mathrm{t}, 0$.

De..nición 2.1.7 El predual de un Semigrupo Dinámico Cuántico T que actúa sobre $B(h)$ es el semigrupo $S$ actuando sobre $\varnothing(h)$ de..nido por:

$$
\operatorname{tr}\left(S_{t}(\Omega)(x)=\operatorname{tr}\left(\Omega \pi_{t}(x)\right)\right.
$$

para cada $\Omega 2 \varnothing(\mathrm{h})$ y x 2 B (h). Ver Teorema 1.2.11, Capítulo 1.

Proposición 2.1.8 Sea T un S.D.C sobre B (h) entonces los siguientes son equivalentes:

1) Existen dos números reales $M, 1$ y $\varnothing, 0$ tal que

$\mathrm{kT}_{\mathrm{t}} \mathrm{k}_{\mathrm{B}(\mathrm{B}(\mathrm{h}))} \cdot \mathrm{M} \exp (\varnothing \mathrm{t})$, para $\mathrm{t}, 0$;

2) El generador in..nitesimal \$ es densamente de..nido y cerrado en la topología ædébil;

3) Si Re $\Pi>$ Øentonces el rango de $\Pi$ i $\$$ coincide con $B(h)$ y se tiene la desigualdad siguiente

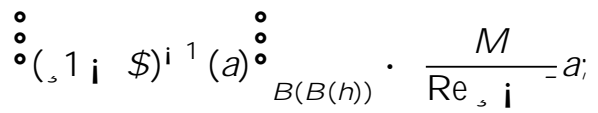

4) El operador resolvente $\left(\Pi_{i} \$\right)^{i}$, está dado por la transformada de Laplace

$$
(\Pi \mathbf{i} \$)^{i 1}(a)={ }_{0}^{Z_{1}} T_{t}(a) \exp (; \Pi) d t
$$

para cada a 2 B (h) y cada número complejo $\Pi$ con $\operatorname{Re} \Pi>\varnothing$. 
Demostración.

Ver. [3] Teorema 3;1;6, págs.166 i 167.

De..nición 2.1.9 Sea E un espacio de Banach con norma jj:j: Un semigrup $T: E$ ! $E$ es fuertemente continuo si $T_{t} \pi \underset{t !}{j i j}{ }_{0}^{j} \pi$, y es uniformemente continuo

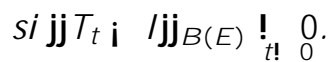

Proposición 2.1.10 Sea $T_{t}$ un semigrupo de operadores acotados sobre un espacio de Banach $\mathrm{E}$ con norma jj:jj:

Los siguientes son equivalentes:

1) La transformación lineal t ! $T_{t}$ es uniformemente continua;

2) La transformación lineal t ! $T_{t}$ es uniformemente diferenciable;

3) El generador in..nitesimal \$ es un operador acotado y

$$
T_{t}={ }_{n, 0} \frac{t^{n}}{n !} \$^{n}
$$

donde la serie converge en la norma de operadores para cada t real.

Si estas condiciones son satisfechas entonces $T$ puede ser extendido a un grupo uniformemente continuo de operadores sobre E que satisface

$$
k T_{t} k_{B(E)} \cdot \exp j t j k \$ k_{B(E)}:
$$

Demostración. Seguimos [3],P rop.3.1.6 pág.166.

3) implica 2)

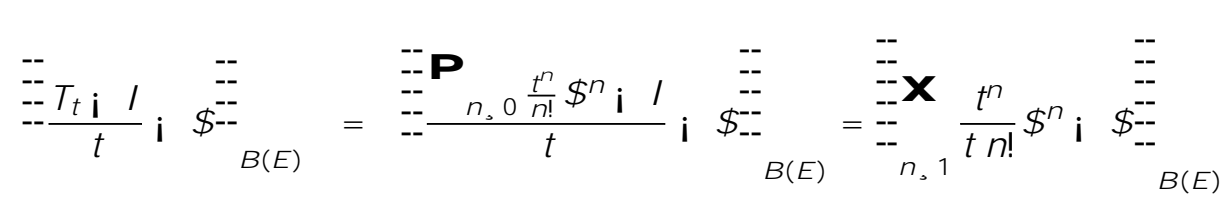


2.1.

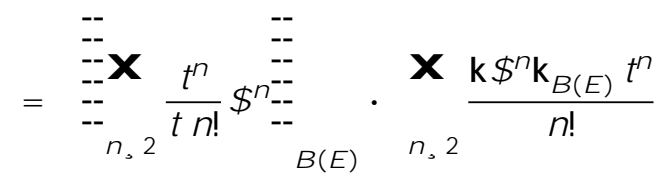

$$
\begin{aligned}
& \text {. } X \frac{k \$ k_{B(E)}^{n_{i} 2} k \$ k_{B(E)}^{2} t^{n_{i} 2} t^{2}}{n !} \\
& \text { n, } 2 \\
& =t^{2} k \$ k_{B(E)}^{2}{ }_{n, 2}^{x} \frac{k \$ k^{n_{i} 2} k \$ k^{2} t^{n_{i} 2}}{n\left(n_{i} 1\right)\left(n_{i} 2\right) !} \\
& \text { - } t^{2} k \$ k_{B(E)}^{2}{ }_{n, 2}^{X} \frac{k \$ k_{B(E)}^{n_{i} 2} k \$ k_{B(E)}^{2} t^{n_{i} 2}}{(n i 2) !} \\
& =t^{2} k \$ k_{B(E)}^{2}{ }_{k, 0}^{X} \frac{k \$ k_{B(E)}^{k} t^{k}}{k !} \\
& =t^{2} j j \$ j j_{B(E)}^{2} e^{t k \$ k} t_{t} 0 .
\end{aligned}
$$

Así T es uniformemente diferenciable.

2) implica 1)

$$
j j T_{t} i \quad T_{0} j j_{B(E)}=\frac{\overline{-}}{-T_{t} i T_{0}} \frac{\overline{-}}{t}{ }_{B(E)} t_{t} !{ }_{0} j j \$ j j B(E)(0)=0
$$

porque

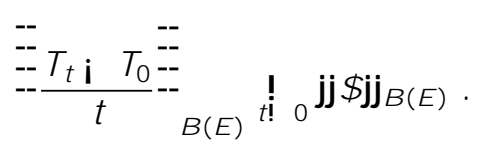

Así resulta que T es uniformemente continuo.

1) implica 2)

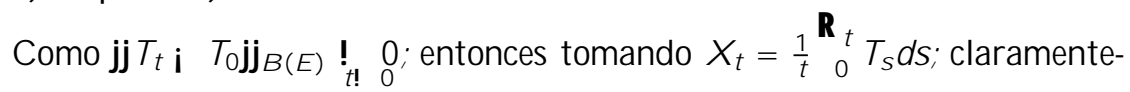
se tiene que $j \mathrm{j} \mathrm{X}_{\mathrm{t}} \mathrm{i} \mathrm{T}_{0} \mathrm{jj}_{\mathrm{B}(\mathrm{E})} !_{\mathrm{t} !} \quad 0$; existe $\pm>0$ tal que si $0<\mathrm{t}<\mathrm{s}$ entonces$j j X_{t} i \quad j j_{B(E)}<1$ con lo cual resulta que $X_{t}=\frac{1}{t}{ }_{0}^{R_{t}} T_{s} d s$ es invertible, acota- 
do y tiene inverso acotado

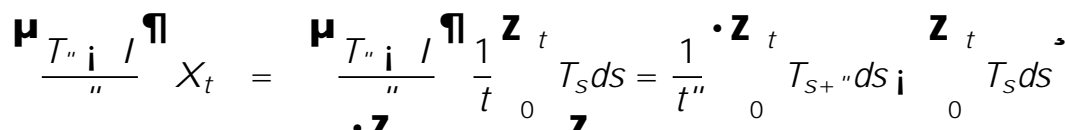

$$
\begin{aligned}
& =\frac{1}{\mathrm{t}^{\prime \prime}}{ }^{\cdot \mathrm{Z}_{\mathrm{t}+"}} \mathrm{Z}_{\mathrm{t}+"} \mathrm{~T}_{\mathrm{s}} \mathrm{ds} \mathrm{i}_{\mathrm{t}} \mathrm{Z}_{\mathrm{s}}^{0} \mathrm{ds}
\end{aligned}
$$

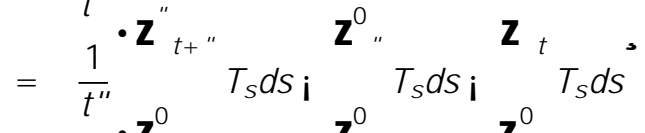

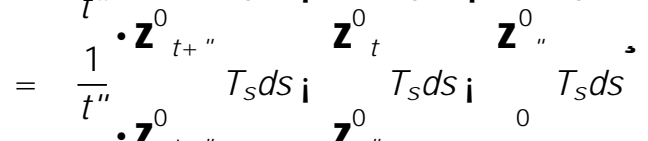

$$
\begin{aligned}
& =\frac{1}{\mathrm{t}^{\prime \prime}} \cdot \mathrm{Z}_{\mathrm{t}+\mathrm{Z}^{\mathrm{t}}}^{0} \mathrm{~T}_{\mathrm{s}} \mathrm{ds} \mathrm{i}^{\mathrm{l}} \mathrm{Z}^{0}{ }^{0} \mathrm{~T}_{\mathrm{s}} \mathrm{ds} \text {, } \\
& =\frac{1}{\mathrm{t}^{\prime \prime}} \quad \mathrm{T}_{\mathrm{s}+\mathrm{t}} \mathrm{ds} \mathrm{i} \quad \mathrm{T}_{\mathrm{s}} \mathrm{ds} \\
& =\frac{\mu}{T_{t} i I^{9}}{ }^{1}{ }^{Z}{ }_{0} T_{s} d s=\frac{1}{t}\left(T_{t} i \quad I\right) X "
\end{aligned}
$$

por lo tanto se tiene que

$$
\lim _{0} \frac{T_{n} i}{11}=\frac{1}{t}\left(T_{t} ; \quad I\right) X_{t}^{i} 1
$$

es decir

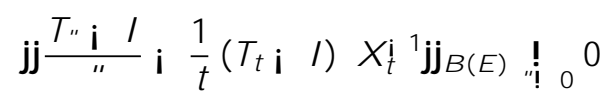

resultando así que T" es uniformemente diferenciable para todo " , 0 :

2) implica 3)

Denotemos por \$ el generador de $T$ ", entonces \$ resulta acotado y además por la parte 2 ) se tiene que $\$=\frac{1}{t}\left(T_{t} i \quad I\right) X_{t}^{i 1}$ de donde obtenemos que

$$
\begin{aligned}
\$ X_{t} & =\frac{T_{t} i}{t} \\
t \$ X_{t} & =T_{t} I^{\prime} Z_{t} \\
T_{t} & =1+\${ }_{0} T_{s} d s:
\end{aligned}
$$

Iterando se obtiene que

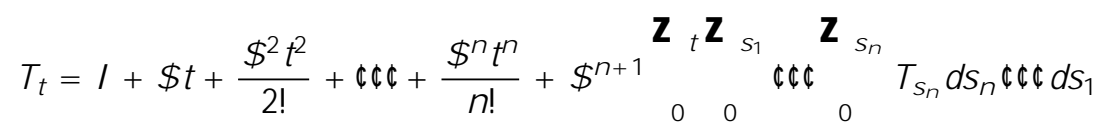

el último elemento de ésta suma se puede estimar porque existen $M, 1$ 
y $\varnothing, 0$ tales que $j j T_{t} j_{B(E)} \cdot M e^{\emptyset t}$, por lo tanto

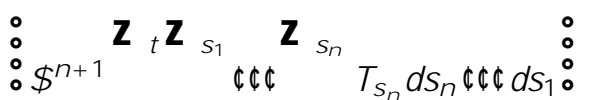

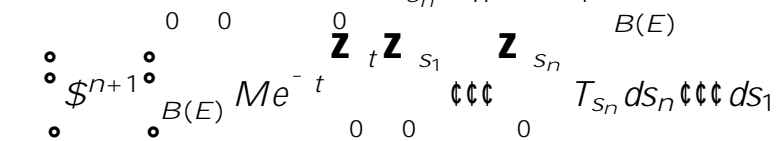

$$
\begin{aligned}
& =\frac{{ }^{\circ} \$^{n+1^{\circ}} B(E) M e^{\varnothing t} t^{n+1}}{n !} ! 0_{0}^{0}
\end{aligned}
$$

de donde concluimos que $T_{t}=P_{n=0} \frac{t^{n} \$^{n}}{n !}$ :

Proposición 2.1.11 Sea $\mathrm{fT}_{t} g_{t}$ o un semigrupo cualquiera(no necesariamente dinámico cuántico) uniformemente continuo de operadores acotados en $B(h)$ con generador in..nitesimal \$. Las siguientes condiciones son equivalentes:

1) $T_{t}$ es ædébil continuo para cada $t, 0$;

2) \$ es ædébil continuo.

Demostración. Seguimos [8], pág.37.

1) implica 2)

Como $T_{t}$ e I son ædébil continuos entonces $\frac{T_{t i} \mid}{t}$ es ædébil continuo y $\$=\mid m_{t !} \frac{T_{t i} \mid}{t}$ en la norma de $B(B(h))$, con lo cual resulta que $\$$ es ædébil continuo, pues el conjunto de los operadores ædébil continuo es cerrado bajo la topología ædébil.

2) implica 1)

Como \$ es ædébil continuo entonces cualquier potencia de éste es ædébilcontinuo, así se tiene que

$$
T_{t}=e^{t \$}=\operatorname{lm}_{m !} \prod_{1} x_{n=0}^{m} \frac{(t \$)^{n}}{n !}=x_{n=0}^{x} \frac{t^{n} \$^{n}}{n !}
$$

es el límite de una sucesión de sumas parciales de operadores ædébil continuos, con lo cual $T_{t}$ resulta ædébil continuo.

Proposición 2.1.12 Sea $\$$ un operador acotado sobre $B(h)$ tal que $\$\left(a^{\circledR}\right)=$ $(\$(a))^{\alpha}$ para cada a 2 B (h). Los siguientes son equivalentes:

1) Para todo a 2 B (h) y t, 0

$$
\exp (t \$)\left(a^{\infty}\right) \exp (t \$)(a) \cdot \exp (t \$)\left(a^{\infty} a\right) ;
$$

2) Para todo a 2 B (h) $a^{x} \$(a)+\$\left(a^{x}\right) a \cdot \$\left(a^{x} a\right)$ : 
Demostración. 1) implica 2)

$$
\begin{aligned}
& \exp (t \$)\left(a^{x}\right) \exp (t \$)(a) ; a^{x} a \cdot \exp (t \$)\left(a^{x} a\right) ; a^{x} a \\
& \frac{e^{t \$} a^{x} e^{t \$} a i a^{x} e^{t \$} a+a^{x} e^{t \$} a_{i} a^{x} a}{t} \cdot \frac{e^{t \$}\left(a^{x} a\right) i a^{x} a}{t} \\
& \frac{{ }^{i} e^{t \$} a^{\infty} i a^{x} e^{t \$} a+a^{\alpha} e^{t \$} a_{i} a^{\phi}}{t} \cdot \frac{e^{t \$} a^{x} a i a^{x} a}{t}
\end{aligned}
$$

por lo tanto cuando t ! 0

$$
a^{a} \$(a)+\$\left(a^{a}\right) a \cdot \$\left(a^{a} a\right):
$$

2) implica 1)

Observemos primero que para a; b2 B (h), tal que $a b=0$, se tiene lo siguiente:

$b^{a} \$\left(a^{a} a\right) b, \quad b^{a} a^{a} \$(a b)+b^{a} \$\left(a^{a}\right) a b=(a b)^{a} \$(a) b+b^{a} \$\left(a^{a}\right)(a b)=0$

Demostraremos que $\left(\Pi_{i} \$\right)^{i}{ }^{1}$ es no negativo para cada $\Pi>k \$ k_{B(B(h))}$, para ello se demostrará que si a $2 \mathrm{~B}(\mathrm{~h})$ es autoadjunto tal que $\left(\Pi_{\mathrm{i}} \$\right)(\mathrm{a})$ es no negativo entonces a es no negativo. Sea $a=x_{i}$ y su descomposición en la parte positiva y negativa. Claramente se tiene que $x y=0$ y usando la observación de arriba se obtiene que $\mathrm{y} \$(\mathrm{x})$ y , 0, así también se tiene que:

$$
\begin{aligned}
0 . & y^{i i} 1_{i} \Pi^{1^{1}} \$(a)^{\Phi} y \\
= & i y^{3} i \Pi^{1} y \$(x) y+\Pi^{1} y \$(y) y \\
& =i y^{3}+\Pi^{1} y \$(y) y
\end{aligned}
$$

por lo tanto,

$$
0 \cdot \mathrm{i}^{3}+\Pi^{1}{ }^{1} \mathrm{y} \$(\mathrm{y}) \mathrm{y}
$$

con lo cual,

$$
y^{3} \cdot \quad \Pi^{1} y \$(y) \text { y y } j j y j j^{3} \cdot \Pi^{i}{ }^{1} j j \$ j j_{B}(B(h)) ~ j j y j j^{3}
$$

ya que $\Pi^{1}{ }^{1} \mathrm{k} \$ \mathrm{k}<1$ se obtiene que $\mathrm{y}=0$; así $\mathrm{a}=\mathrm{x}$ es no negativo. A hora usando el hecho de que $\mathrm{e}^{\mathrm{t} \$}$ es positivo y lineal se tiene que,

$$
e^{t \$}\left(a^{x}\right)={ }^{i} e^{t \$}(a)^{\phi_{x}}
$$




$$
\begin{aligned}
& \text { por lo tanto, } \\
& \qquad \begin{array}{c}
\frac{d^{3}}{d s} e^{\left(t_{i} s\right) \$} e^{s \$}\left(a^{\infty}\right) e^{s \$}(a) \\
=e^{\left(t_{i} s\right) \$}{ }_{i}^{f} \$^{i} e^{s \$}\left(a^{\infty}\right) e^{s \$}(a)^{\phi}+\$ e^{s \$}\left(a^{\infty}\right) e^{s \$}(a)+e^{s \$}\left(a^{\infty}\right) \$ e^{s \$}(a)^{\alpha} \\
\cdot a^{\infty} \$(a)+\$\left(a^{\alpha}\right) a:
\end{array}
\end{aligned}
$$

integrando esta desigualdad sobre el intervalo $[0 ; t](t, 0)$ se obtiene que $\exp (t \$)\left(a^{a}\right) \exp (t \$)(a) \cdot \exp (t \$)\left(a^{a} a\right):$

De..nición 2.1.13 Un operador acotado \$ sobre B (h) es Ilamado condicionalmente completamente positivo, si para cada $n$, 1 la transformación lineal $\$^{n}$ sobre $B(h)-M_{n}$ de..nido por:

$$
\$^{n}\left(a-E_{i j}\right)=\$(a)-E_{i j}(1 \cdot i ; j \cdot n)
$$

satisface la desigualdad

$$
\$^{n}\left(x^{x} x\right) \text { i } x^{x} \$^{n}(x) \text {; } \$^{n}\left(x^{x}\right) x+x^{x} \$^{n}(1) x, 0
$$

para cada $\times 2 \mathrm{~B}(\mathrm{~h})-\mathrm{M}_{\mathrm{n}}$.

Proposición 2.1.14 Sea $T$ un semigrupo uniformemente continuo sobre $B(h)$ con generador in..nitesimal $\$$. E ntonces $T_{t}$ es completamente positivo para cada $\mathrm{t}$, 0 si y sólo si $\$$ es condicionalmente completamente positivo y $\$\left(a^{\mathbb{\alpha}}\right)=$ $(\$(a))^{x}$ para cada a 2 B (h) :

Demostración. Seguimos [8], págs.39-40.

Sea T un semigrupo dinámico cuántico. Claramente con la notacion de los $T_{t ; n}$ para cada $T_{t}, f T_{t}^{n} g_{t, o}$ resulta ser un semigrupo uniformemente continuo en $B(h)-M_{n}$. A hora usando la Ecuación $(1 ; 2)$ del Capítulo 1 se tiene que

$$
T_{t}^{n}\left(x^{x}\right)\left(T_{t}^{n}(1)\right)^{i}{ }^{1} T_{t}^{n}(x) \cdot T_{t}^{n}\left(x^{x} x\right) ; x 2 B(h) \text { y t, } 0
$$

al derivar esta desigualdad en $\mathrm{t}=0$ se obtiene que,

$$
\$^{n}\left(x^{a} x\right) \text { i } x^{a} \$^{n}(x) \text { i } \$^{n}\left(x^{a}\right) x+x^{a} \$^{n}(1) x, 0
$$

resultando así que \$ es condicional completamente positivo.

Para ver que $\$\left(a^{\infty}\right)=(\$(a))^{\infty}$ obsérvese que

$$
\begin{aligned}
\$\left(a^{a}\right) & =\lim _{t !} \frac{T_{t}\left(a^{a}\right) ; a^{a}}{t}=\lim _{t !} \frac{T_{t}(a) ; a^{9_{a x}}}{t} \\
& =(\$(a))^{a}
\end{aligned}
$$

pues la operación * es continua en la norma de $B(h)$.

Recíprocamente, supongamos primero que $\$(1) \cdot 0$; pues de no ser 
el caso así, es su..ciente considerar el operador $\${ }_{1}=\$$ i c; donde $\mathrm{C}=\mathrm{k} \$(1) \mathrm{k}_{\mathrm{B}(\mathrm{h})}$ : Claramente se tiene que la E cuación $(2 ; 1)$ se cumple tanto para $\$$ y $\$ 1$; así $T_{t}=e^{t \$}=e^{c t+t \$ 1}=e^{t c} e^{t \$ 1}$; resultando que $T_{t}$ es completamente positivo si $\mathrm{e}^{\mathrm{t} \$ 1}$ lo es. A sí pues supóngase que $\$(1) \cdot 0$ :

Entonces para cada $\mathrm{n}, 1$; la Desigualdad $(2 ; 10)$ dice que

$$
\begin{aligned}
& x^{a} \$^{n}(x)+\$^{n}\left(x^{a}\right) x \cdot \$^{n}\left(x^{a} x\right) \\
& \mathrm{e}^{t \$^{n}}\left(x^{\mathbb{d}}\right) \mathrm{e}^{t \$^{n}}(x) \quad \cdot \quad \mathrm{e}^{t \$^{n}}\left(x^{\mathbb{d}} x\right) \text {; por la ecuación 2;8) }
\end{aligned}
$$

así los $T_{t}^{n}$ son positivos con lo cual los $T_{t}$ son completamente positivos.

Lema 2.1.15 Sea \$ : B (h) ! B (h) condicionalmente completamente positivo. Entonces para cada $n, 1$ y $a_{1} ;:: ; a_{n} 2 A ; u_{1} ;:: ; u_{n} 2 h$, tal que ${ }_{j=1}^{n} a_{j} u_{j}=$ 0 , se tiene lo siguiente

$$
x_{i ; j=1}^{n} h u_{i} ; \$\left(a_{i}^{x} a_{j}\right) u_{j} i, 0:
$$

Demostración. Seguimos [8], pág.40.

Sea $\times 2 B(h)-M_{n}$ que tenga la forma

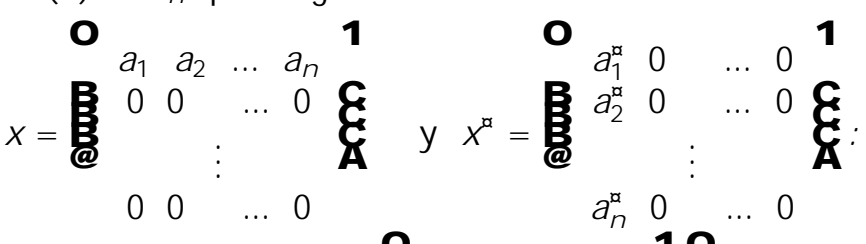

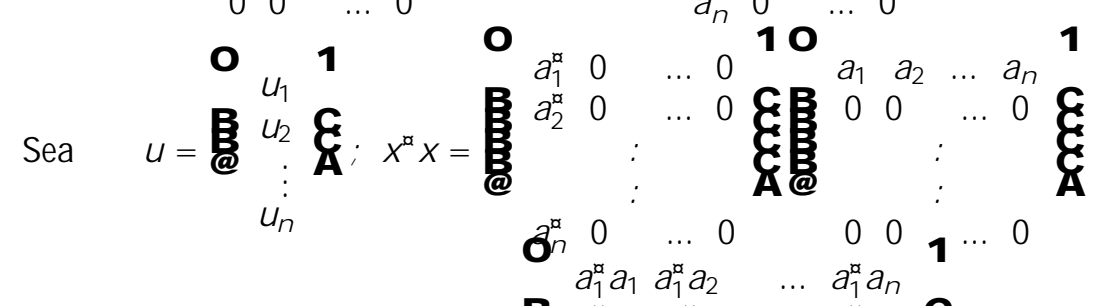

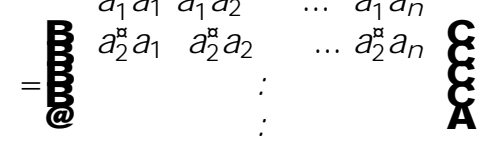

$$
\begin{aligned}
& a_{n}^{\alpha} a_{1} \quad a_{n}^{\alpha} a_{2} \quad \ldots \quad a_{n}^{\alpha} a_{n}
\end{aligned}
$$

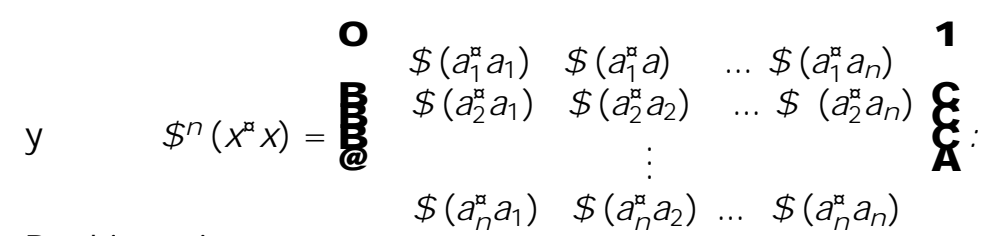

Por hipótesis tenemos que:

0 . hu; $\left(\$^{n}\left(x^{a} x\right)\right.$ i $x^{a} \$^{n}(x)$ i $\left.\$^{n}\left(x^{a}\right) x+x^{a} \$^{n}(1) x\right)$ ui
$=$ hu; $\$^{n}\left(x^{\natural} x\right)$ ui i hxu; $\$^{n}(x)$ ui i hu; $\$^{n}\left(x^{a}\right) x u i+h u ; \$^{n}(1) x u i$ : 
Por separado se puede demostrar que en esta desigualdad el único término que no es 0 es

es decir $0 \cdot h u ; \$^{n}\left(x^{a} x\right)$ ui $=\stackrel{h u ; \$^{n}\left(x^{a} x\right) u i ;}{n}, h_{i} ; \$\left(a_{i}^{a} a_{j}\right) u_{j} i$.

Teorema 2.1.16 Sea \$: B (h)! B (h) operador lineal con la propiedad $\$\left(a^{\mathbb{a}}\right)=$ $(\$(a))^{\infty}$ para cada a 2 B (h) : \$ es condicionalmente completamente positivo si y sólo si existe una transformación completamente positiva $i$ en $B(h)$ y un operador G 2 B (h) tal que para cada a 2 B (h)

$$
\$(x)=i(a)+G^{x} a+a G:
$$

A demás $G$ satisface la desigualdad $G+G^{\alpha} \cdot \$(1)$ :

Demostración. Seguimos [8], págs.40-42.

Supongamos que $\$(a)=i(a)+G^{x} a+a G$. La linealidad de $\$$ es obvia y es claro que $\$\left(a^{\infty}\right)=(\$(a))^{\infty}$ por la positividad de $\mathbf{i}$. A hora veremos que esta transformación \$ es condicionalmente completamente positiva. Fijemosun $n$ entero positivo y denotemos por $G_{n}$ al operador $G-1$ en $B(h)-M_{n}$. Para cada $\times 2$ B ( $\mathrm{h})-\mathrm{M}_{\mathrm{n}}$ tenemosque

$$
\$^{n}(x)=i^{n}(x)+G_{n}^{\alpha} x+x G_{n}:
$$

A hora usando la Desigualdad $(2 ; 10)$ obtenemos que:

$$
i^{n}\left(x^{\alpha} x\right) i i^{n}\left(x^{\alpha}\right) x i x^{\alpha} i^{n}(x)+x^{\alpha} i^{n}(1) x, 0:
$$

Ya que $\mathbf{i}$ es completamente positiva, entonces $i^{n}$ es positiva para cada $n, 1$ y como para cada $t, 0 e^{t_{i}{ }^{n}}$ es positivo, concluimos que $e^{t_{i}}$ es completamente positivo. Se sigue de la desigualdad $(2 ; 10)$ que i condicionalmente completamente positivo. Por la igualdad arriba de $\$^{\mathrm{n}}$ tenemos que $\$$ es condicionalmente completamente positivo.

R ecíprocamente, supóngase que \$ escondicionalmente completamente positivo y que $\$\left(a^{a}\right)=(\$(a))^{x}$ con a $2 \mathrm{~B}(\mathrm{~h})$. Fijemos un vector unitario $¥ 2 \mathrm{~h}$ y consideremos el operador $\mathrm{G}$ en $\mathrm{h}$ con su adjunto de..nido por

$$
\left.G^{x} u=\$(j u i h ¥ j) ¥ i \quad \frac{1}{2} h ¥ ; \text { (j¥i } ¥ j\right) ¥ i u
$$

para cada u $2 \mathrm{~h}$. A hora para cada $\mathrm{n}, 1$ y $\mathrm{a}_{1} ;:: ; ; \mathrm{a}_{\mathrm{n}} 2 \mathrm{~A}, \mathrm{u}_{1} ;:: ; \mathrm{u}_{\mathrm{n}} 2 \mathrm{~h}$ hagamos $u_{n+1}=¥, \int=i \quad{ }_{j=1}^{n} a_{j} u_{j}, a_{n+1}=j \int i h \neq j:$ Con estas notaciones tenemos que

$$
x_{j=1}^{x+1} a_{j} u_{j}={ }_{j=1}^{X^{n}} a_{j} u_{j}+a_{n+1} u_{n+1}={ }_{j=1}^{X^{n}} a_{j} u_{j}+j \int i h \neq j \neq
$$




$$
={ }_{j=1}^{X} a_{j} u_{j}+h \supsetneqq ; ¥ i \int={ }_{j=1}^{X} a_{j} u_{j}+@_{i}{ }_{j=1}^{n} a_{j} u_{j} A=0
$$

con lo cual ahora aplicando la desigualdad $(2 ; 11)$;

$$
\begin{aligned}
& 0 \cdot{ }_{i ; j=1}^{x+1} h u_{i} ; \$\left(a_{i}^{\alpha} a_{j}\right) u_{j} i \\
& =\sum_{i ; j=1}^{X n} h u_{i} ; \$\left(a_{i}^{\alpha} a_{j}\right) u_{j} i+{ }_{i=1}^{x+1} h u_{i} ; \$\left(a_{i}^{\alpha} a_{n+1}\right) u_{n+1} i+ \\
& { }^{x+1}-u_{n+1} ; \$^{i} a_{n+1}^{\alpha} a_{j}{ }^{\phi} u_{j}{ }^{\circledR}{ }^{i}-u_{n+1} ; \$ a_{n+1}^{\alpha} a_{n+1}{ }^{\phi} u_{n+1}{ }^{\circledR} \\
& j=1 \\
& =x_{i ; j=1}^{n} h u_{i} ; \$\left(a_{i}^{\alpha} a_{j}\right) u_{j} i+{ }_{i=1}^{X n} h u_{i} ; \$\left(a_{i}^{\alpha} a_{n+1}\right) u_{n+1} i+
\end{aligned}
$$

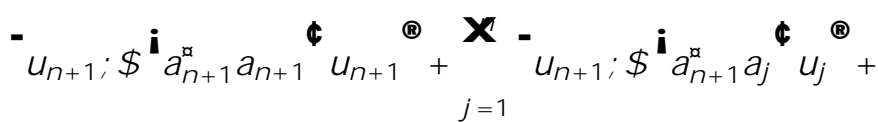

$$
\begin{aligned}
& -u_{n+1} ; \$ a_{n+1}^{\alpha} a_{n+1}{ }^{\Phi} u_{n+1}{ }^{\circledR} i^{-} u_{n+1} ; \${ }^{i} a_{n+1}^{\alpha} a_{n+1}{ }^{\Phi} u_{n+1}{ }^{\circledR} \\
& =x_{i ; j=1}^{n} h u_{i} ; \$\left(a_{i}^{\alpha} a_{j}\right) u_{j} i+{ }_{i=1}^{X n} h u_{i} ; \$\left(a_{i}^{\alpha} a_{n+1}\right) u_{n+1} i+ \\
& X^{n}-u_{n+1} ; \${ }^{i} a_{n+1}^{x} a_{j} \phi^{\phi} u_{j}{ }^{\circledR}-u_{n+1} ; \${ }^{i} a_{n+1}^{x} a_{n+1}{ }^{\phi} u_{n+1}{ }^{\circledR} \\
& j=1 \\
& =X_{i ; j=1}^{n} h u_{i} ; \$\left(a_{i}^{\alpha} a_{j}\right) u_{j} i+{ }_{i=1}^{n} h u_{i} ; \$\left(a_{i}^{\alpha} j \int i h \supsetneqq j\right) ¥ i+
\end{aligned}
$$

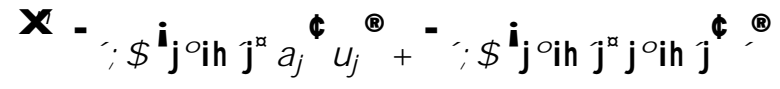

$$
\begin{aligned}
& j=1 \\
& =\sum_{i ; j=1}^{X} h u_{i} ; \$\left(a_{i}^{\alpha} a_{j}\right) u_{j} i+{ }_{i=1}^{X n} h u_{i} ; \$\left(j a_{i}^{\alpha} \int i h \ldots j\right) ¥ i+ \\
& X^{h} \quad h ¥ ; \$\left(j ¥ i h \int j a_{j}\right) u_{j} i+h ¥ ; \$\left(j ¥ i h \int j j \int i h f j\right) ¥ i \\
& j=1 \\
& =\sum_{i ; j=1}^{n} h u_{i} ; \$\left(a_{i}^{\alpha} a_{j}\right) u_{j} i+{ }_{i=1}^{X} h u_{i} ; \$\left(j a_{i}^{\alpha} \int i h f j\right) ¥ i+
\end{aligned}
$$




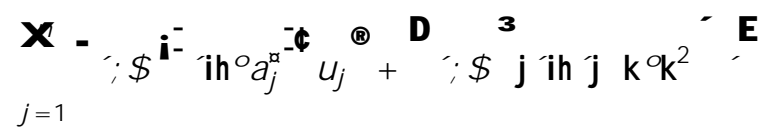

$$
\begin{aligned}
& =x_{i ; j=1}^{n} h u_{i} ; \$\left(a_{i}^{\alpha} a_{j}\right) u_{j} i+X_{i=1}^{n} h u_{i} ; \$\left(j a_{i}^{\alpha} \int i h \supsetneqq j\right) ¥ i+
\end{aligned}
$$

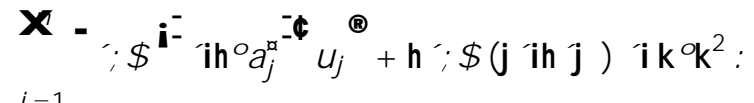

Por lo tanto,

$$
\begin{aligned}
& 0 . x_{i ; j=1}^{X n} h u_{i} ; \$\left(a_{i}^{\alpha} a_{j}\right) u_{j} i+{ }_{i=1}^{X n} h u_{i} ; \$\left(j a_{i}^{\alpha} \int i h f j\right) ¥ i+
\end{aligned}
$$

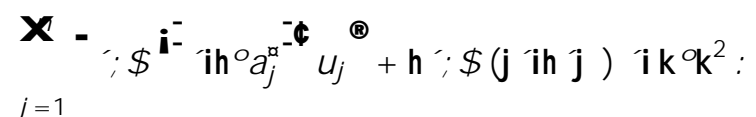

Al recordar la de.nición de $\mathrm{G}^{\mathrm{a}}$; los últimos tres términos se pueden escribir como

$$
\begin{gathered}
X_{i=1}^{n} h u_{i} ; G^{\infty} a_{i}^{\alpha} \int i+{ }_{j=1}^{X n} h G^{\infty} a_{i}^{\alpha} \int ; u_{j} i \\
=i_{i ; j=1}^{X n} h u_{i} ; G^{\infty} a_{i}^{\infty} a_{j} u_{j} i{ }_{i ; j=1}^{X n} h u_{i} ; a_{i}^{\infty} a_{j} G u_{j} i
\end{gathered}
$$

de donde obtenemos la desigualdad

$$
0 \cdot{ }_{i ; j=1}^{X h} h u_{i} ;\left(\$\left(a_{i}^{\alpha} a_{j}\right) i \quad G^{\alpha} a_{i}^{\alpha} a_{j} \quad a_{i}^{\alpha} a_{j} G\right) u_{j} i:
$$

De..namos i de la siguiente forma,

$$
\mathrm{i}(\mathrm{a})=\$(\mathrm{a}) \mathrm{i}^{\mathrm{\alpha}} \mathrm{a} \text { i } \mathrm{aG}
$$

así i es completamente positivo. Finalmente tenemos que,

$$
G+G^{\infty}=\$(1) i \quad i(1) \cdot \$(1)
$$

lo que ..naliza la demostración. 
Teorema 2.1.17 (Lindblad) Un operador acotado \$ sobre B $(h)$ es el generador in..nitesimal de un Semigrupo Dinámico Cuántico uniformemente continuo si y sólo si existen un espacio de Hilbert $\mathrm{K}$; un operador ' $: \mathrm{h}$ ! $\mathrm{h}$ - $\mathrm{K}$ y un operador $\mathrm{G}$ en $\mathrm{h}$ acotado tales que

$$
\$(x)={ }^{x}(x-1)^{\prime}+G^{x} x+x G
$$

para $\times 2$ B (h) : El operador \$ puede ser elegido de tal forma que

$$
f(x-1) ' u: x 2 \text { B (h) ; u } 2 \text { hg }
$$

es total en $\mathrm{h}-\mathrm{K}$.

Demostración.

Sea $T$ semigrupo dinámico cuántico uniformemente continuo. Entonces su generador in..nitesimal \$ es condicionalmente completamente positivo, por lo tanto puede ser presentado como $\$(x)=i(x)+G^{\alpha} x+x G, x 2$ B (h)

(Teorema 2.1.15). Pero, por otra, parte $\mathrm{i}$ es completamente positiva de $B(h)$ a $B(h)$ mismo. A sí usando el Teorema de Stinespring se tiene que existe un espacio de Hilbert $K$ y $L: h$ ! $h$ - $K$ lineal tal que

$$
i(x)=L^{x}(x-1) L
$$

por lo tanto se tiene así, $\$(a)=L^{x}(x-1) L+G^{x} x+x G$ :

Para demostrar el recíproco, supóngase que

$$
\$(x)=L^{x}(x-1) L+G^{x} x+x G
$$

entonces por el Teorema de Krauss, ecuación $(1 ; 2 ; 4), L^{x}(x-1) L$ es æedébil continuo. Entonces $\$$ es condicionalmente completamente positivo y ædébil continuo. A demás para todo $\times 2 \mathrm{~B}(\mathrm{~h})$ se cumple lo siguiente:

$$
\begin{aligned}
& \$\left(x^{\not \alpha}\right)=L^{\not \alpha}\left(x^{\not}-1\right) L+G^{\not \alpha} x^{\not}+x^{\not \alpha} G \\
& =L^{\infty}(x-1)^{\alpha} L+\left(G^{\infty} x\right)^{\alpha}+(x G)^{\infty} \\
& =\left(L^{x}(x-1) L\right)^{x}+\left(G^{x} X+x G\right)^{x} \\
& =(\$(x))^{x}:
\end{aligned}
$$

Claramente se tiene que

$$
\begin{aligned}
& \mathrm{h} f ; \$(1) \int \mathrm{i}=\mathrm{h} f ; \mathrm{L}^{\mathrm{x}}(1) \mathrm{L} \int \mathrm{i}+\mathrm{h} \int ; \mathrm{G}^{\mathrm{x}} \int \mathrm{i}+\mathrm{h} \int ; \mathrm{G} \int \mathrm{i} \\
& =h L \int ; \int i+h \int ; G^{\infty} \int i+h \int ; G \int i \\
& =j j L \int j j^{2}+h \int ; G^{x} \int i+h \int ; G \int i \\
& \text {, } h \int ; G^{\mathfrak{a}} \int i+h \int ; G \int i \text { : }
\end{aligned}
$$

Por lo tanto,

$$
\$(1), G^{\infty}+G \text { : }
$$

Resultando así que \$ es el generador in..nitesimal de un semigrupo dinámico cuántico uniformemente continuo. 
Corolario 2.1.18 (Lindblad-Gorini-Kossakowski-Sudarshan)Si \$ es el generador in..nitesimal de un S.D.C fT $\mathrm{tg}_{\mathrm{t}}$ uniformemente continuopque actúa sobre $\mathrm{B}(\mathrm{h})$ entonces existe una sucesión $f L \cdot g_{=1}^{1}, L \cdot 2 B(h)$ tal que ${ }_{1}^{1}{ }_{=1} L \cdot L \cdot<1$ en la topología fuerte y existe $\mathrm{G} 2 \mathrm{~B}(\mathrm{~h})$ tal que

$$
\$(x)={ }_{`=1}^{x} L^{\alpha} x L \cdot+G^{\alpha} x+x G:
$$

Demostración. A plicando el Teorema de Lindblad se tiene que

$$
\$(x)=i(x)+G^{\alpha} x+x G
$$

con $G 2$ B (h). Por el Teorema de Stinespring, i es completamente positivo y ædébil continuo, y usando el Teorema de Krauss, i $(x)={ }_{:=1}^{1} L{ }^{x} X L$. donde ${ }^{1}=1$ L $L \cdot<1$ en la topología débil. De donde se deduce que

$$
\$(x)={ }_{:=1}^{X} L^{x} x L \cdot+G^{x} x+x G:
$$

Para la construcción del Semigrupo Dinámico Cuántico minimal que en el próximo capítulo se lleva acabo. La ecuación de la parte ii) de la Hipótesis A que se usa en gran parte de ese capítulo resulta ser una condición necesaria para la contracción de este semigrupo minimal; es decir si se tiene que

$$
0 \cdot \mathrm{T}_{\mathrm{t}}^{\min }(\mathrm{I}) \cdot \mathrm{I}
$$

entonces

$$
\begin{aligned}
& T_{t}^{\min }(I) \mathrm{i} \cdot 0 \\
& \frac{\mathrm{T}_{\mathrm{t}}^{\min }(\mathrm{I}) \mathrm{i} \mathrm{I}}{\mathrm{t}} \cdot 0
\end{aligned}
$$

y siendo $\$$ min el generador in..nitesimal de este semigrupo dinámico cuántico minimal, también se cumple que en la topología ædébil(æ )

$$
\$^{\min }(I)=\nsim \lim _{\mathrm{t} ! 0^{+}} \frac{\mathrm{T}_{\mathrm{t}}^{\min }(\mathrm{I}) \mathrm{i} \mathrm{I}}{\mathrm{t}}
$$

de donde resulta así que

$$
\$^{\min }(I) \cdot 0
$$

Por otra parte, más adelante en ese mismo capítulo se demuestra que $D^{1} \$ \min 1 / 2 D(1 / 2)$,

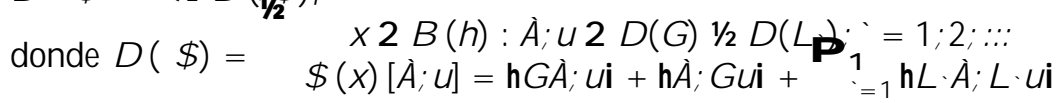

$$
\$(I)[i ; u]=h_{i} ; \$(I) \text { ui }={ }^{-} \int ; \$^{\min }(I) u^{\circledR} \cdot 0
$$


de donde se deduce que

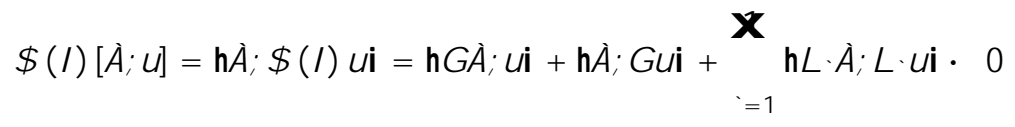

que es la ecuación en la parte ii) de la Hipótesis A.

La fórmula de Leibniz ([1]; pág. 274) del Cálculo elemental será ampliamente usada en egte trabajo, dice la siguiente:

Sea $E=(t ; s) 2 R^{2} j 0 \cdot s \cdot t$ y sea $f: E$ ! $R$ continua tal que $\frac{@}{@ f} f$ es una función continua entonces

$$
\frac{@}{(d)}_{0}^{Z_{t}} f(t ; s) d s=f(t ; t)+{ }_{0}^{Z_{t}} \frac{@}{@} f(t ; s) d s: \quad(2 ; 13)
$$




\section{Capítulo 3}

\section{El Semigrupo Dinámico Cuántico M inimal (S.D.C.M.)}

Este capítulo es el más importante de todo el trabajo, debido a que es aquí donde se presenta por primera vez a la Ecuación de Lindblad, cuya solución es única cuando el semigrupo dinámico cuántico minimal que la resuelve es conservativo. Para garantizar la existencia de una solución se lleva acabo la construcción del semigrupo dinámico cuééntic@ minimal (S.D.C.M .) Teorema 3;1;7; el cual es denotado por $\mathrm{T}^{\mathrm{mm}}=\mathrm{T}_{\mathrm{t}}^{\mathrm{mm}}$ t, o y que no necesariamente es conservativo. Por último el Corolario 3;1;8, muestra que el semigrupo dinámico cuántico minimal cuando es conservativo es la única solución de la Ecuación de Lindblad. EI resultado más importante de este capítulo el cual proporciona un criterio para la conservatividad de este semigrupo minimal y que usaremos posteriormente en los ejemplos del Capítulo 5; es el Teorema 3;2;7. 


\subsection{Hipótesis $A$}

i) Sea G el generador in..nitesimal de un semigrupo de contracciones fuertemente continuo $\mathrm{fP}_{\mathrm{t}} \mathrm{g}_{\mathrm{t}, \mathrm{o}}$ en $\mathrm{h}$ :

ii) Sean $f \mathrm{~L} \cdot \mathrm{g}^{1}=1$ operadores no necesariamente acotados de tal forma que $\mathrm{D}(\mathrm{G}) \mathrm{t}_{2}^{1 / 2} \mathbb{1}_{=1}^{1} \mathrm{D}(\mathrm{L} \cdot)$ y para cada u $2 \mathrm{D}(\mathrm{G})$

$$
h u ; G u i+h G u ; u i+{ }_{=1}^{X} h \cdot u ; L \cdot u i \cdot 0:
$$

Lema 3.1.1 Supóngase que se tienen las Hipótesis A. Entonces para cada - 1 las siguientes condiciones son equivalentes:

1) La transformación $t$ ! L $\cdot P_{t} u$ es continua en la norma de $h$ para cada u 2 $\mathrm{D}(\mathrm{G})$ :

2) La transformación $t$ ! $L \cdot P_{t} u$, es derivable en la norma para cada $u 2$ $D^{1} \mathrm{G}^{2} \mathrm{y}$

$$
\frac{d}{d t} L \cdot P_{t} u=L \cdot P_{t} G u:
$$

3) :La transformación $t$ ! $P_{1}{ }_{1} \mathrm{kAE} \cdot \mathrm{P}_{\mathrm{t}} u \mathrm{k}_{h}^{2}$, es derivable para cada $\mathrm{u} 2$ $D^{1} G^{2}$ y $Æ 2 \quad B(h)$ : Es decir se tiene lo siguiente

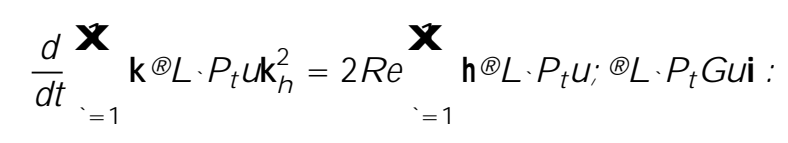

Demostración. Seguimos [8], págs.44.

Para demostrar 1 ), bastará ver que si s ! 0 entonces

$$
k L \cdot P_{t+s} u_{i} L \cdot P_{t} u k_{h}^{2}=k L \cdot\left(P_{t+s} u_{i} P_{t} u\right) k_{h}^{2} ! 0:
$$

Para verlo, haremos uso de la Hipótesis $A$ y el hecho de que $D(G)$ es invariante bajo la acción de $\mathrm{P}_{\mathrm{t}}$

$$
\begin{aligned}
& k L \cdot\left(P_{t+s} u_{i} P_{t} u\right) k_{h}^{2} \cdot{ }_{=1}^{X} k L \cdot\left(P_{t+s} u_{i} P_{t} u\right) k_{h}^{2} \\
& ={ }_{=1}^{X} L \cdot\left(P_{t+s} i P_{t}\right) u ; L \cdot\left(P_{t+s} u_{i} P_{t}\right) u i \\
& \text { - i } 2 R \operatorname{Reh}\left(P_{t+s} \text { i } P_{t}\right) u ;\left(P_{t+s} \text { i } P_{t}\right) \text { Gui }{ }_{s ! 0} 0
\end{aligned}
$$


porque $\mathrm{fP}_{\mathrm{t}} \mathrm{g}_{\mathrm{t}, 0}$ es fuertemente continuo.

A sí tenemos que $L \cdot P_{t+s} u{ }_{s} ! o b \cdot P_{t} u:$

Para probar 2), observemos que debido a que $\mathrm{D}^{\mathrm{i}} \mathrm{G}^{2}{ }^{\Phi}$ es invariante bajo

$\mathrm{fP}_{\mathrm{t}} \mathrm{g}_{\mathrm{t}, 0}$, tenemos por la Hipótesis A

$$
\begin{aligned}
& \stackrel{\circ}{\circ} \frac{\stackrel{0}{\circ} \cdot\left(\left(P_{t+s} i P_{t}\right) u\right)}{s} \text { i L.P.P } \stackrel{\circ}{\circ} \stackrel{\circ}{\circ} \\
& \text {. } \quad i 2 \operatorname{Re} e^{i} \frac{\left(P_{t+s} i P_{t}\right) u}{s} ; P_{t} G u ; G \frac{\left(P_{t+s} i P_{t}\right) u}{s} \text { i } P_{t} G u^{\text {ๆ }}
\end{aligned}
$$

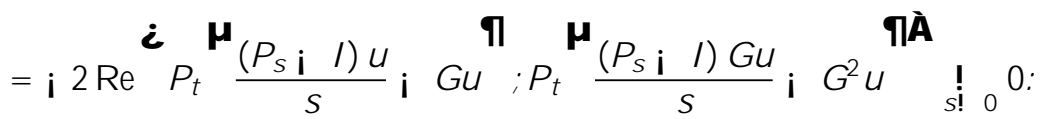

A quí hemos hecho uso de que si u $2 \mathrm{D}^{\mathrm{i}} \mathrm{G}^{{ }^{\Phi}} ; \mathrm{Gu} 2 \mathrm{D}(\mathrm{G})$ y

$$
\frac{\left(P_{s} i \quad \text { I }\right) G u}{s} \sum_{s ! 0} G^{2} u
$$

por lo tanto tenemos que:

$$
\frac{d}{d t} L \cdot P_{t} u=L \cdot P_{t} G u:
$$

La prueba de 3) es como sigue: 
44CAPÍTULO 3. EL SEMIGRUPO DINÁMICO CUÁNTICO MINIMAL (S.D.C.M.)

$$
\begin{aligned}
& \text { Sean s; t , } 0 \text {, u } 2 \mathrm{D}^{\mathrm{i}} \mathrm{G}^{2^{\Phi}} \text { y } Æ 2 \mathrm{~B}(\mathrm{~h}) \text {; entonces } \\
& \text { X } \\
& \text { k但. } P_{t+s} u k_{h}^{2} \\
& =1
\end{aligned}
$$

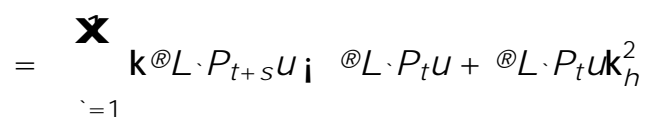

$$
\begin{aligned}
& ={ }_{=1}^{X} k \text { 且 } \cdot\left(P_{t+s} \text { i } P_{t}\right) u+A \text { 且 } \cdot P_{t} u k_{h}^{2}
\end{aligned}
$$

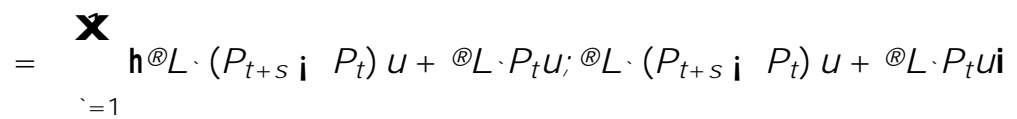

$$
\begin{aligned}
& ={ }_{=1}^{X} h \text { AE } \cdot\left(P_{t+s} \text { i } P_{t}\right) u ; A \text { A } \cdot\left(P_{t+s} \text { i } P_{t}\right) u i+
\end{aligned}
$$

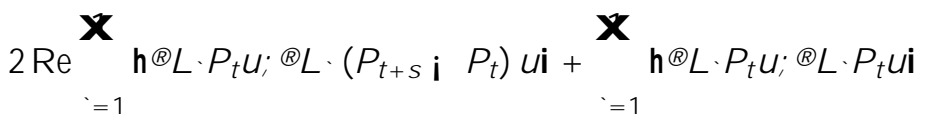

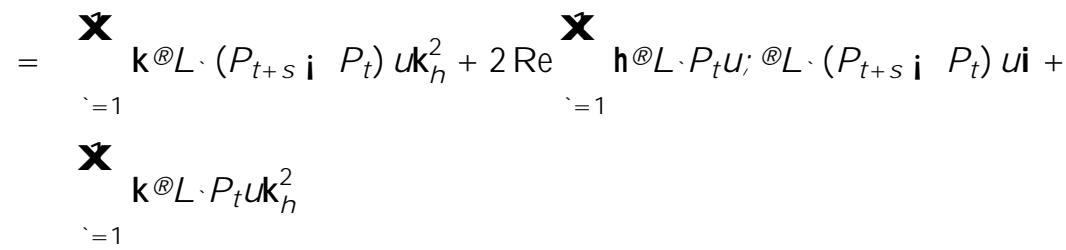

Por lo tanto tenemos que

$$
\begin{aligned}
& { }_{=1}^{X} k \text { AE } \cdot P_{t+s} u k_{h}^{2} i^{X} k \text { AEI } \cdot P_{t} u k_{h}^{2}
\end{aligned}
$$

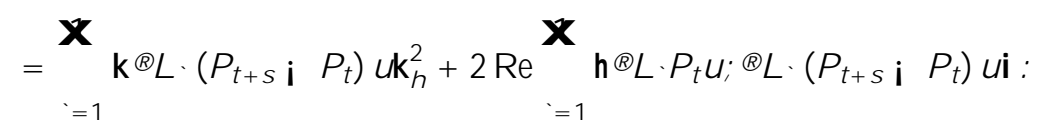

A hora desarrollemos

$$
\begin{aligned}
& 2 R e^{X} h \text { hEE } \cdot P_{t} u \text {; 伍 } \cdot\left(P_{t+s} \text { i } P_{t}\right) u i
\end{aligned}
$$

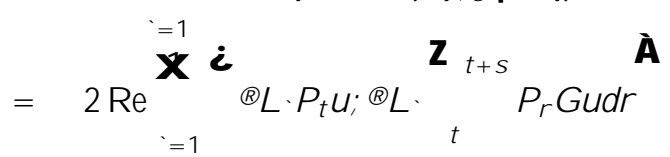

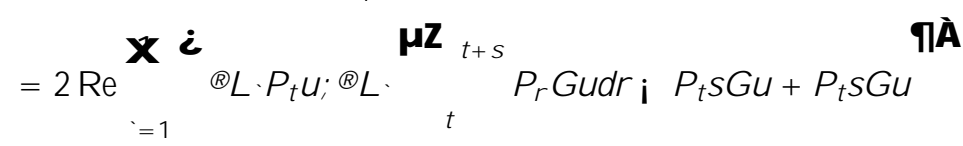




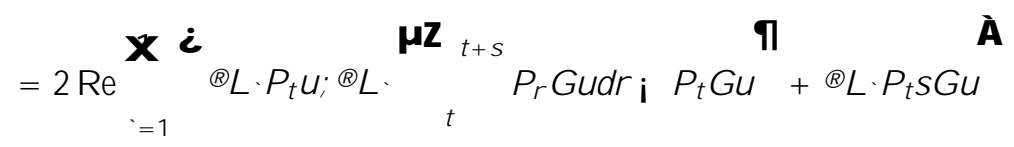

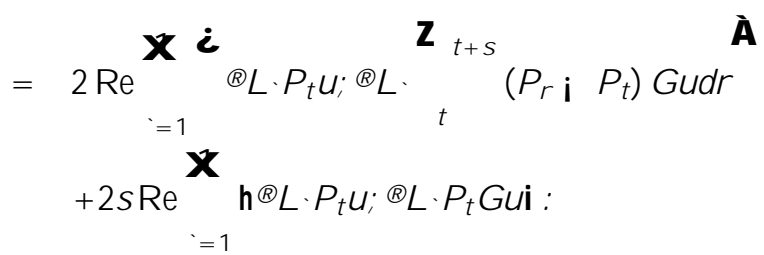

De donde podemos ver que:

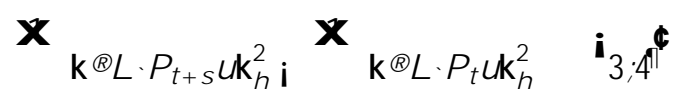

$$
\begin{aligned}
& =\underbrace{X^{`}=1}_{=1} \mathrm{kAE} \cdot\left(\mathrm{P}_{\mathrm{t}+\mathrm{s}} \mathrm{i} \mathrm{P}_{\mathrm{t}}\right) \mathrm{uk}_{\mathrm{h}}^{\mathrm{n}}+ \\
& 2 R e^{X{ }^{X} i} \text { AE } \cdot P_{t} u ; \text { AE }{ }^{Z_{t+s}}\left(P_{r} \text { i } P_{t}\right) \text { Gudr } \\
& +2 \mathrm{sRe} e_{=1}^{X} h \& E \cdot P_{t} u ; \text { AE } \cdot P_{t} G \text { ui : }
\end{aligned}
$$

De la última expresión, analicemos cada sumando acotándolos si es necesario con la Hipótesis A y la desigualdad (3;2):

$$
\begin{aligned}
& P_{{ }^{1}=1} k \text { 但} \cdot\left(P_{t+s} \text { i } P_{t}\right) u k_{h}^{2} \cdot P_{{ }^{1}=1} k A k_{1}^{2} k L \cdot\left(P_{t+s} \text { i } P_{t}\right) u k_{h}^{2} \\
& \text { - i } 2 \text { kAR }_{1}^{2} \operatorname{Reh}\left(P_{t+s} \text { i } P_{t}\right) u ;\left(P_{t+s} \text { i } P_{t}\right) \text { Gui } \\
& \text {. } 2 k \Leftrightarrow k_{1}^{2} k\left(P_{t+s} \text { i } P_{t}\right) u k_{h} k\left(P_{t+s} \text { i } P_{t}\right) G u k_{h}
\end{aligned}
$$

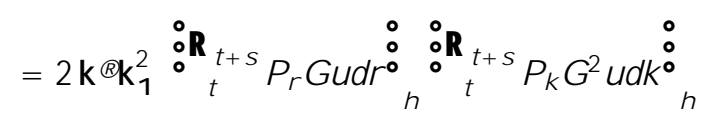


46CAPÍTULO 3. EL SEMIGRUPO DINÁMICO CUÁNTICO MINIMAL (S.D.C.M.)

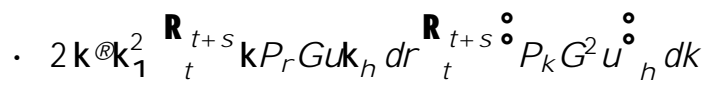

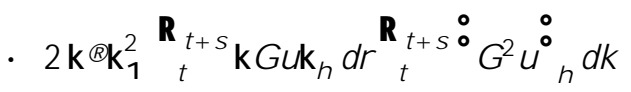

$$
\begin{aligned}
& =2 \mathrm{kAH}_{1}^{2} \mathrm{kGuk}_{\mathrm{h}}{ }^{\circ} \mathrm{G}^{2} \mathrm{o}_{\mathrm{h}}^{\circ} \mathrm{s}^{2}:
\end{aligned}
$$

Es decir, hemos probado que:

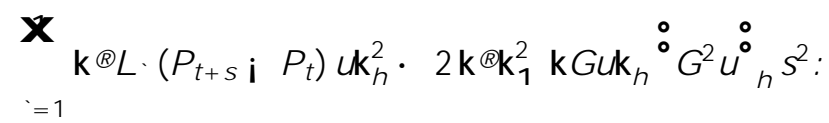

Para el segundo sumando, dividimos entre s;

$$
\begin{aligned}
& \frac{2 R^{P} e_{i=1}^{D} \text { AE. } P_{t} u ; \text { 但 }{ }_{t}^{R_{t+s}}\left(P_{r} ; P_{t}\right) \text { Gudr }}{S} \\
& =2 R e^{X i} A \mathbb{E} \cdot P_{t} u ; A E \cdot \frac{1}{S}{ }_{t}^{Z_{t+s}}\left(P_{r} \text { i } P_{t}\right) \text { Gudr }
\end{aligned}
$$

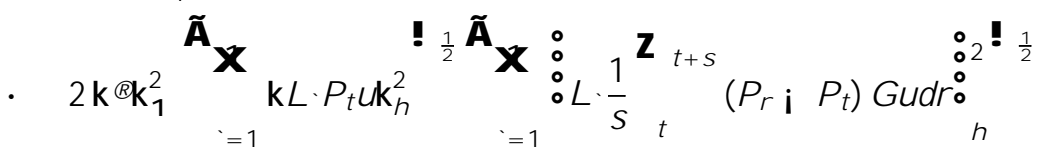

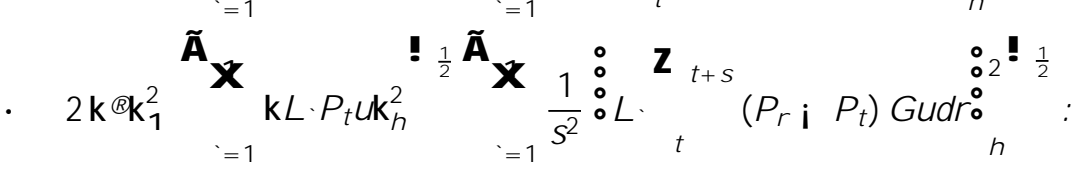

De donde tenemos que:

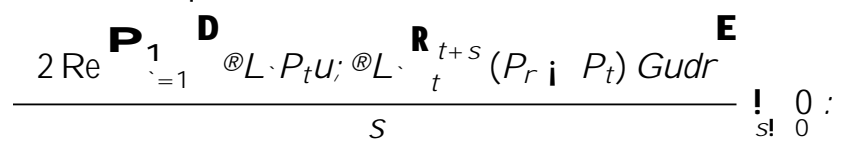

Inmediatamente dividiendo la ecuación ${ }^{\mathrm{i}} 3 ; 5^{41^{\natural}}$ por s tenemos que:

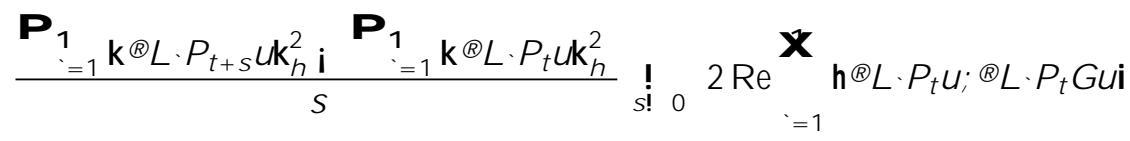


lo que demuestra la parte 3).

A hora consideraremos la forma sesquilineal \$: B (h) $f D(G) f D(G)$ dada por la siguiente regla:

$$
\$(x)[u ; i]=h u ; x G_{i} i+h G u ; x i i+{ }_{=1}^{x} h \cdot u ; x L \cdot i i
$$

claramente \$ es lineal en el operador $x$ y es sesquilineal en $D(G) £ D(G)$ con las condiciones de la Hipótesis A. \$ se le llama el generador formal, generador de Lindblad o Lindbladiano.

Nuestra meta es construir un Semigrupo Dinámico Cuántico que satisfaga la siguiente ecuación, dados u; ¿ 2 D (G)

$$
h u ; T_{t}(x) i i=h u ; x_{i} i+{ }_{0}^{Z_{t}} \$\left(T_{s}(x)\right)[u ; i] d s
$$

o en forma equivalente

$$
\frac{d}{d t} h ; T_{t}(x) i i=\$\left(T_{t}(x)\right)[u ; i]
$$

con valor inicial hu; $T_{t}(x) \sum i j_{t=0}=h u ; x i i$ : Esta última ecuación es llamada Ecuación de Lindblad.

De.nición 3.1.2 Sea a operador lineal densamente de..nido(es decir con dominio denso) en un espacio de $B$ anach $\varnothing$ y $Q$ subespacio vectorial de $\varnothing$ entonces $Q$ es esencia de a si $Q \frac{1}{1 / 2} D(\underline{a})$ y para cada $\times 2$ D ( a $)$ existe $f x_{n} g_{n=1}^{1} 1 / 2 Q$ tal que $x_{n}{ }_{n ! 1} x y \underline{a}\left(x_{n}\right)_{n !}{ }_{1}^{a}(x)$ :

Proposición 3.1.3 Supóngase que se satisface la Hipótesis $A$ y que $\times 2$ $B(h)$ : Sea $f_{t}(x) g_{t, o}$ una familia de elementos de $B(B(h))$ ædébil continua respecto al tiempo tal que

$$
\mathrm{kT}_{\mathrm{S}}(\mathrm{x}) \mathrm{k}_{1} \cdot \mathrm{kxk}_{1}:
$$

Entonces las siguientes condi $\mathrm{R}_{t}$ nes son equivalentes:

1) $\mathrm{hu} ; \mathrm{T}_{\mathrm{t}}(\mathrm{x}) \dot{\mathrm{i}}=\mathrm{hu} ; \mathrm{x}_{\dot{\mathrm{i}}} \mathrm{i}+{ }_{0}^{\mathrm{t}} \$\left(\mathrm{~T}_{\mathrm{s}}(\mathrm{x})\right)[\mathrm{u} ; \dot{i}] \mathrm{ds}$; se tiene para cada $\mathrm{u} ; \dot{i} 2$ $D(G)$;

2) Para cada $u ; i 2 D(G)$ tenemos que

$h u ; T_{t}(x) i i=h P_{t} u ; P_{t} x_{i} i+{ }_{=0}^{x^{A}} Z_{t} h \cdot P_{t_{i} s} u ; T_{s}(x) L \cdot P_{t_{i}}$ si $i d s$ 
Demostración. Seguimos [8], pág.46.

1) implica 2)

Tomemos t , 0 ..jo, entonces para ¿; u 2 D (G)

$$
\begin{aligned}
& \frac{d}{d s} h P_{t_{i} s} u ; T_{s}(x) P_{t_{i} s} i \\
& =h_{i} G P_{t_{i} s} u ; T_{s}(x) P_{t_{i} s} i i+\$\left(T_{s}(x)\right)\left[P_{t_{i} s} u ; P_{t_{i} s} \dot{ }\right]+ \\
& \mathrm{HP}_{\mathrm{t}_{\mathrm{i}} s} \mathrm{u} ; \mathrm{T}_{\mathrm{s}}(\mathrm{x})\left(\mathrm{i} \mathrm{GP}_{\mathrm{t}_{\mathrm{i}} \mathrm{s}} \dot{\mathrm{C}}\right) \mathrm{i} \\
& =h_{i} G P_{t_{i} s} u ; T_{s}(x) P_{t_{i} s} i i+h P_{t_{i} s} u ; T_{s}(x)\left(i G P_{t_{i} s} \dot{ }\right) i \\
& +h G P_{t_{i} s} u ; T_{s}(x) P_{t_{i} s} \dot{i}+h P_{t_{i} s} u ; T_{s}(x) G P_{t_{i} s} \dot{i} \\
& +{ }^{X} H \cdot P_{t_{i} s} u ; T_{s}(x) L \cdot P_{t_{i} s i} \\
& ={ }_{=1}^{X} h \cdot P_{t_{i} s} u ; T_{s}(x) L \cdot P_{t_{i} s} i^{i}
\end{aligned}
$$

es decir tenemos que,

$$
\frac{d}{d s} h P_{t_{i} s} u ; T_{s}(x) P_{t_{i} s} \dot{i}={ }_{=1}^{x} h \cdot P_{t_{i} s} u ; T_{s}(x) L \cdot P_{t_{i} s} i i:
$$

A hora integrando esta última igualdad sobre $[0 ; t]$; deducimos lo siguiente:

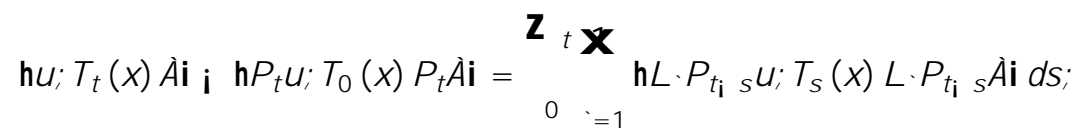

con lo cual

$$
h u ; T_{t}(x) i i=h P_{t} u ; x P_{t} i i+{ }_{=1}^{x}{ }^{Z} h \cdot P_{t i s} u ; T_{s}(x) L \cdot P_{t_{i}} \text { s } i i d s:
$$


2) implica 1)

Sean $\mathrm{u} ;$ i $2 \mathrm{D}^{\mathrm{i}} \mathrm{G}^{2}{ }^{\natural}$ y $\mathrm{T}_{\mathrm{s}}(\mathrm{x}), 0$, usaremos las ecuaciones $(12 ; 12)$ y $(3 ; 7)$ al igual que la identidad de polarización compleja( [3]; pág. 38).

$\frac{d}{d t} h u ; T_{t}(x) i i=\frac{d}{d t} h P_{t} u ; x P_{t} i i+{ }_{`=1}^{x} Z_{t} h \cdot P_{t_{i} s} u ; T_{s}(x) L \cdot P_{t_{i}} z_{i} i d s:(3 ; \not l)$

Como $T_{s}(x), 0$ entonces existe y $2 B(h)$ tal que $y^{\alpha} y=T_{s}(x):$ Por lo tanto,

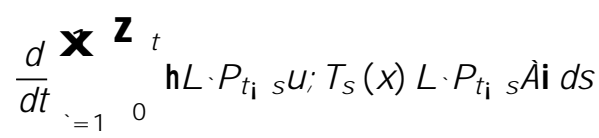

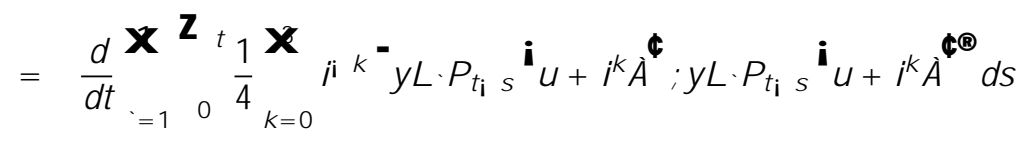

así se obtiene que

$$
\begin{aligned}
& \frac{d}{d t} h u ; T_{t}(x) i i=h P_{t} G u ; x P_{t} i i+h P_{t} u ; x P_{t} G_{i} i
\end{aligned}
$$

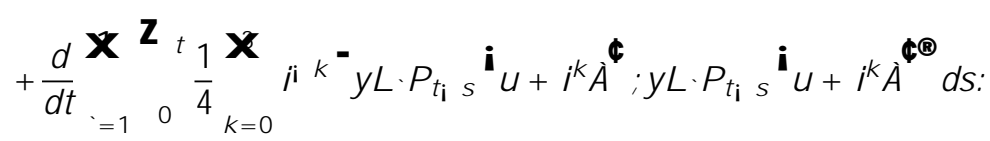

Pero desarrollando el segundo término de esta última igualdad se tiene que este es igual a

$$
\begin{aligned}
& { }^{X}{ }_{=1}^{1} X_{k=0}^{3} i i^{\circ}{ }^{\circ} y L \cdot P_{t_{i} t}{ }^{i} u+i^{k}{ }_{i}^{\phi_{02}^{\circ}} h
\end{aligned}
$$

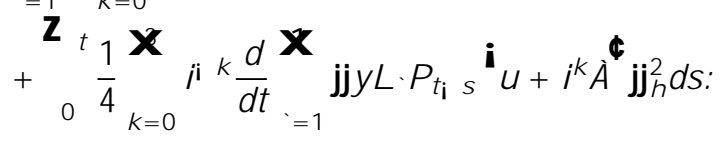

Así, por la Ecuación $(3 ; 4)$ tenemos que la última expresión en el extremo derecho de la última igualdad es igual a,

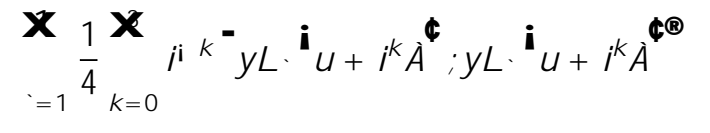

$$
\begin{aligned}
& +{ }_{0}^{Z_{t}} \frac{1}{4}_{k=0}^{X^{\beta}} i^{i k} 2 R e^{X}-y L \cdot P_{t_{i} s}{ }^{i} u+i^{k}{ }_{i}^{\phi} ; y L \cdot P_{t_{i} s} G^{i} u+i^{k}{ }_{i}{ }^{\circledR \circledast} d s ;
\end{aligned}
$$


50CAPÍTULO 3. EL SEMIGRUPO DINÁMICO CUÁNTICO MINIMAL (S.D.C.M.)

por lo tanto,

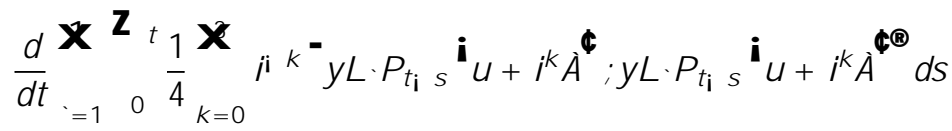

$$
\begin{aligned}
& ={ }_{=1}^{X} H \cdot u ; T_{S}(x) L \cdot i^{i}
\end{aligned}
$$

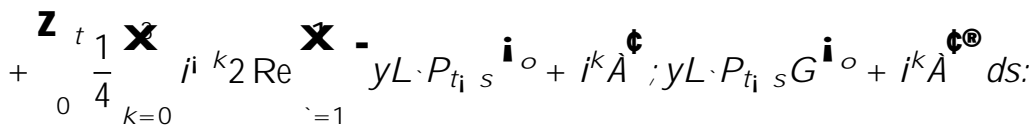

Sin embargo, al desarrollar

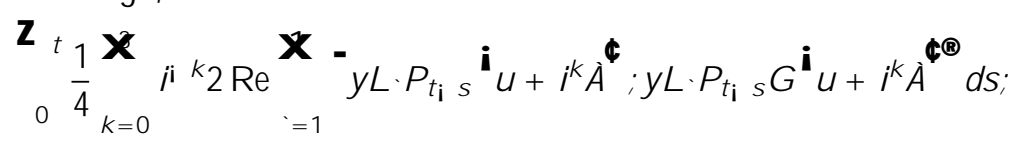

Se tiene que es igual a

$$
\begin{aligned}
& Z_{{ }^{\prime}=1}^{X^{X}} \frac{1}{4}_{k=0}^{X^{3}} i^{i k^{-}} y L \cdot P_{t_{i} s} G^{i} u+i^{k}{ }_{i}^{\Phi} ; y L \cdot P_{t_{i} s}{ }^{i} u+i^{k}{ }_{i}^{d \circledR} d s
\end{aligned}
$$

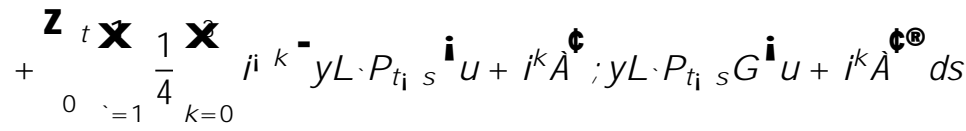

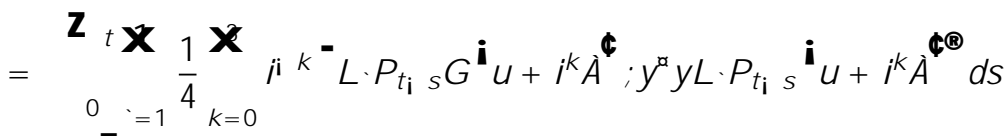

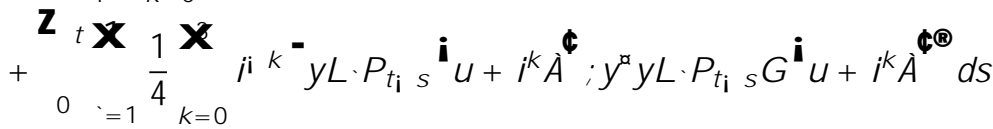

$$
\begin{aligned}
& =\sum_{Z^{\prime}=1}^{Z} L \cdot P_{t_{i} s} G u ; T_{s}(x) L \cdot P_{t_{i} s} i d d s \\
& +{ }_{0 \cdot=1}^{Z_{t}^{i}=1} L \cdot P_{t_{i} s} U_{;} T_{s}(x) L \cdot P_{t_{i} s} G_{i} i d s
\end{aligned}
$$

Por lo tanto,

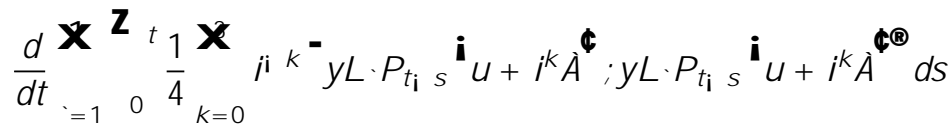

$$
\begin{aligned}
& ={ }_{v=1}^{X} L \cdot u ; T_{s}(x) L \cdot i i+{ }_{0 \cdot=1}^{Z} L \cdot P_{t_{i} s} G u ; T_{s}(x) L \cdot P_{t_{i}} s i i d s+ \\
& Z_{t} \times \\
& L \cdot P_{t_{i} s} u ; T_{s}(x) L \cdot P_{t_{i} s} G_{i} i d s \\
& 0{ }^{`}=1
\end{aligned}
$$


Poniendo todas estas igualdades en $(3 ; \not 7)$

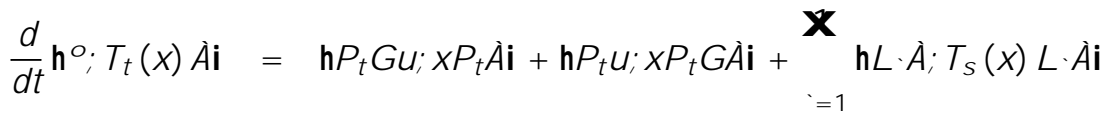

$$
\begin{aligned}
& +Z_{0=1}^{Z_{t} X} h \cdot P_{t_{i} s} u ; T_{s}(x) L \cdot P_{t_{i} s} G_{i} i d s \\
& +{ }_{0 \cdot=1}^{Z_{t} X^{X}} h \cdot P_{t_{i} s} G u ; T_{s}(x) L \cdot P_{t_{i} s} \text { i i ds: }
\end{aligned}
$$

Al sumar el primer término y el quinto se obtiene que es igual a

$$
h u ; T_{t}(x) G_{i} i \text {; }
$$

análogamente la suma del segundo témino con el cuarto nos da

$$
h G u ; T_{t}(x) i i \text { : }
$$

Por lo tanto,

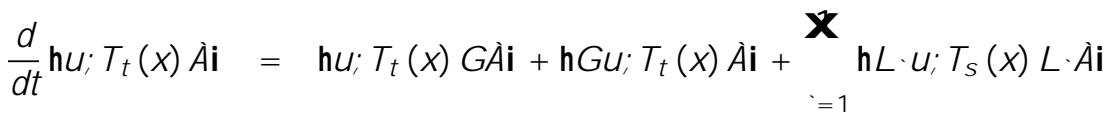

$$
\begin{aligned}
& =\mathrm{hu} ; \$ T_{t}(x) i \mathrm{i} ;
\end{aligned}
$$

es decir,

$$
\frac{d}{d s} h u ; T_{t}(x) i i=h u ; \$ T_{s}(x) i i
$$

al integrar sobre el intervalo $[0 ; t]$;

$$
\begin{aligned}
Z_{t} \frac{d}{d s} h u ; T_{t}(x) i i d s & ={ }_{0}^{Z_{t}} \$\left(T_{s}(x)\right)[u ; i] d s \\
\text { hu; } T_{t}(x) i i \text { i hu; } x_{i} i & ={ }_{0}^{Z_{t}} \$\left(T_{s}(x)\right)[u ; i] d s ;
\end{aligned}
$$

de donde se sigue que,

$$
h ; T_{t}(x) \dot{i}=h u ; x_{i} i+{ }_{0}^{Z_{t}} \$\left(T_{s}(x)\right)[u ; i] d s:
$$

Ya que $D^{i} G^{2}{ }^{\Phi}([20]$, Lema 3;2, 3;3, págs.9 i 10) es una esencia de $G$, la demostración vale en todo $D(G)$ : 
Proposición 3.1.4 Supóngase que se tiene la Hipótesis $A$. De.namos para cada $t, 0$; la siguiente sucesión de transformaciones lineales de $B(h)$ en si mismo;

$$
\int ; T_{t}^{0}(x) i^{\circledR}=h P_{t} \int ; x P_{t} i \mathrm{i}
$$

Suponiendogque se ha de.nido $T_{t}^{n} p a r a$ algún $n 2 N$, se de..ne $T_{t}^{n+1}$

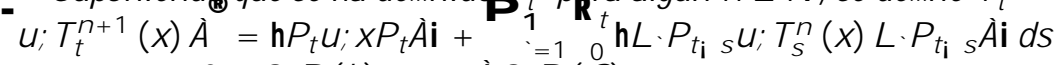
para t, 0 ; $x 2 \mathrm{~B}(\mathrm{~h})$; y u; $i 2 \mathrm{D}(\mathrm{G})$ :

Entonces para cada t, 0 y para cada $\mathrm{n} 2 \mathrm{~N}$ se tiene:

1) $T_{t}^{n}$ es contracción, lineal, completamente positivo y normal.

2) Para cada $n 2 \mathrm{~N}$ y cada $\times 2 \mathrm{~B}(\mathrm{~h})$ la transformación $t$ ! $\mathrm{T}_{\mathrm{t}}^{\mathrm{n}}(\mathrm{x})$ es ædébil continuo.

3) La sucesión $f T_{t}^{n}(x) g_{n, o}$ es creciente para cada $x 2$ B (h) que sea positivo.

4) $T_{t}^{n}(1) \cdot 1$ para cada $t, 0$ y cada $n, 0$ :

Demostración. Seguimos [8], págs.47.

Claramente la transformación $\mathrm{T}_{\mathrm{t}}^{0}$, está bien de..nida y tiene todas las propiedades arriba mencionadas. Sólo falta ver que $\mathrm{T}_{\mathrm{t}}^{0}(1) \cdot 1$ : Observemos que

$$
\begin{aligned}
-\mathrm{u}_{;} \mathrm{T}_{\mathrm{t}}^{0}(1) \mathrm{u}^{\circledR}= & h \mathrm{P}_{\mathrm{t}} \mathrm{u} ; 1 \mathrm{P}_{\mathrm{t}} \mathrm{ui}=h \mathrm{P}_{\mathrm{t}} \mathrm{u} ; \mathrm{P}_{\mathrm{t}} \mathrm{ui}=k \mathrm{P}_{\mathrm{t}} u k_{\mathrm{h}}^{2} \cdot k P_{\mathrm{t}} k_{\mathrm{B}(\mathrm{h})}^{2} k u k_{h}^{2} \\
\cdot & k u k_{\mathrm{h}}^{2}=h \mathrm{~h} ; 1 \mathrm{ui}
\end{aligned}
$$

lo cual implica que

$$
\mathrm{T}_{\mathrm{t}}^{0}(1) \cdot 1 \text { : }
$$

Supongamos que los $T_{t}^{n}$ son contracciones sobre $B(h)$ y que satisfacen la primera condición para un $n 2 \mathrm{~N}$..jo. Entonces demostraremos que $T_{t}^{n+1}$ tiene las mismas propiedades.

Para cada $\times 2$ B (h) positivo y $\int$; i $2 \mathrm{D}(\mathrm{G})$, la integral en el lado derecho de $(3 ; 8)$ está bien de..nida por las propiedades de los $T_{t}^{n}$ y además por la positividad de éste mismo, se tiene que

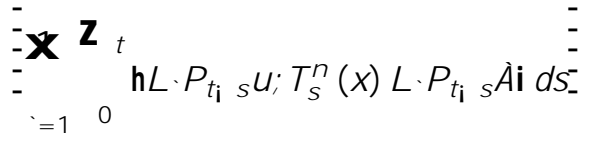

$$
\begin{aligned}
& { }^{\mathrm{A}} \mathrm{Z}_{\mathrm{t}} \\
& `=1 \quad 0 \\
& \mathrm{X}_{\mathrm{t}}
\end{aligned}
$$

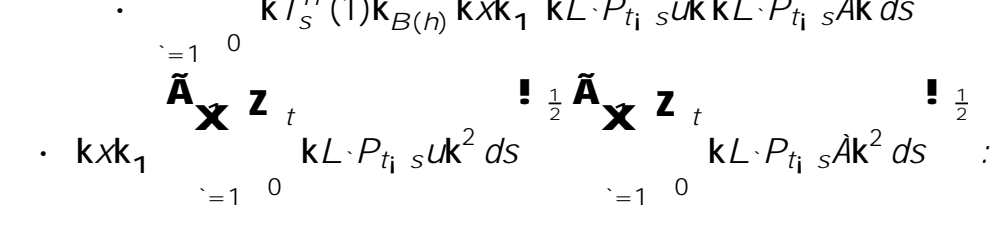

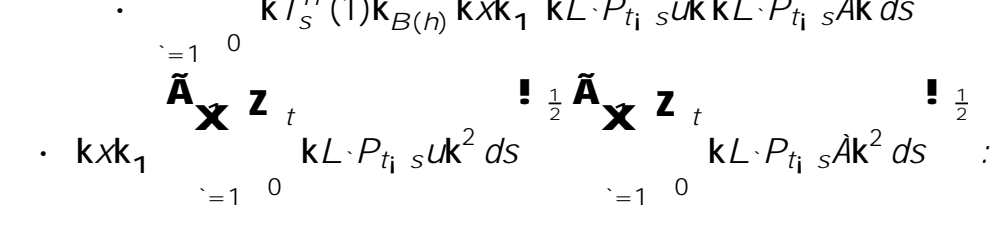

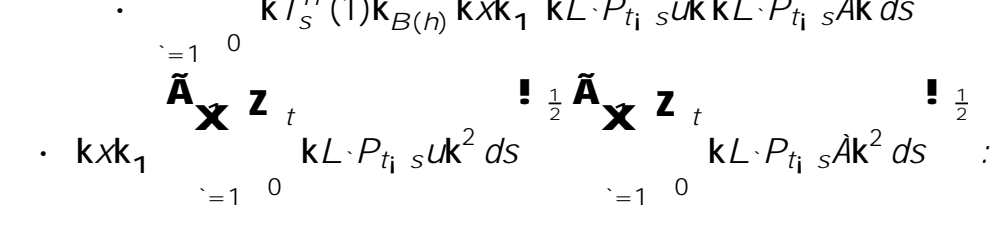

$$
\begin{aligned}
& k T_{s}^{n}(x) k_{B(h)} k L \cdot P_{t_{i}} \text { ukkL } \cdot P_{t_{i}} \text { s } i k d s \\
& k T_{s}^{n}(1) k_{B(h)} k x k_{1} k L \cdot P_{t_{i}} s u k k L \cdot P_{t_{i}} \text { s } i k d s
\end{aligned}
$$


Usando la desigualdad $(3 ; 2)$ obtenemos que

$$
\begin{aligned}
& { }^{2} \mathrm{Z}_{\mathrm{t}} \quad \mathrm{Z}_{\mathrm{t}} \\
& `=1 \quad 0 \\
& k L \cdot P_{t_{i} s} u k^{2} d s \quad \text { i } 2 R e r P_{t_{i} s} u ; G P_{t_{i} s} \int i d s \\
& =\mathrm{Z}_{\mathrm{t}} \frac{\mathrm{d}}{\mathrm{ds}} \mathrm{k} \mathrm{P}_{\mathrm{t}_{\mathrm{i}} \mathrm{s}} u k_{\mathrm{h}}^{2} \mathrm{ds} \\
& =\mathrm{kuk}_{\mathrm{h}}^{2} \mathrm{i} \mathrm{kP}_{\mathrm{t}} \mathrm{uk} \mathrm{k}_{\mathrm{h}}^{2} \text { : }
\end{aligned}
$$

A nalogamente:

$$
\begin{aligned}
& x^{2} Z_{t} \\
& =1 \quad 0 \\
& k L \cdot P_{t_{i} s} i k^{2} d s=k i k_{h}^{2} i \quad k P_{t} i k^{2}
\end{aligned}
$$

por lo tanto,

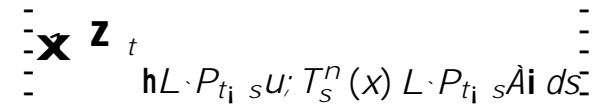

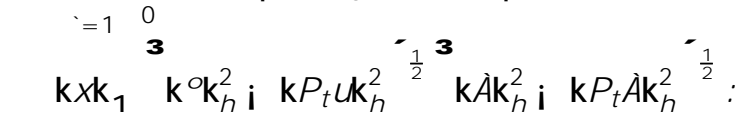

Recordemos la siguiente propiedad

$a, b, c, d$ implica que ${ }^{i} a^{2} i b^{2^{\phi_{1}}} i^{2} c^{2} d^{d^{\phi_{1}}} \cdot a c i$ bd:

Tenemos así que:

$$
\begin{aligned}
& \overline{-x^{2}} Z_{\mathrm{t}} \\
& =10 \\
& h \cdot P_{t_{i} s} u ; T_{s}^{n}(x) L \cdot P_{t_{i} s i} i d s_{-}^{-} \cdot k_{k x}\left(k u k_{h}: k_{i} k_{h} i k P_{t} u k_{h}: k P_{t} i k_{h}\right) ;
\end{aligned}
$$

como además

$$
j h P_{t} u ; x P_{t} i j \cdot k P_{t} u k_{h}: k P_{t} i k_{h} k x k_{1} ;
$$

se tiene que,

${ }^{-} u ; T_{t}^{n+1}(x) i^{\mathbb{B}} \cdot k P_{t} u k_{h}: k P_{t} i k_{h} k x k_{1}+k x k_{1}\left(k u k_{h}: k i k_{h} ; \quad k P_{t} u k_{h}: k P_{t} i k_{h}\right):$

$$
\text { Por lo tanto, } \quad=-u_{;} T_{t}^{n+1}(x) i^{\circledR} \cdot k^{\circledR} k_{h}: k_{i} k_{h} k x k_{1} \text { : }
$$

A hora veamos que $T_{t}^{n+1}$ es contracción.

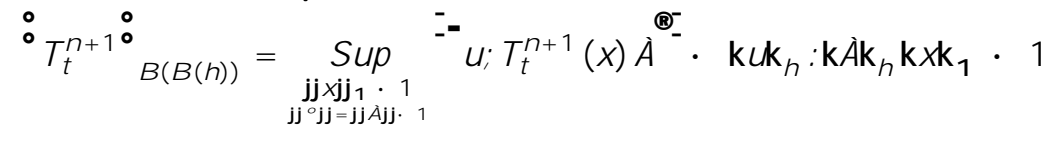

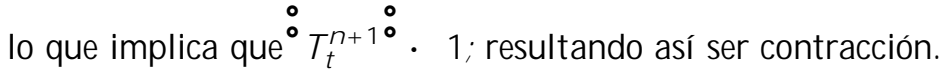




\section{CAPÍTULO 3. EL SEMIGRUPO DINÁMICO CUÁNTICO MINIMAL (S.D.C.M.)}

Enseguida probamos la normalidad de $T_{t}^{n+1}$ : Es claro que $T_{t}^{0}$ es normal y completamente positivo pues tiene la forma de K raus.

Supongamos que se ha establecido que $T_{t}^{n}$ es normal se probará que $T_{t}^{n+1}$ también es normal.

Tomemos $i 2 D(G)$ y $f x_{A E} g_{F E}$ una red creciente de elementos positivos en $B(h)$ tal que $x_{K E} ! a_{w} \times 2 B(h)$ :

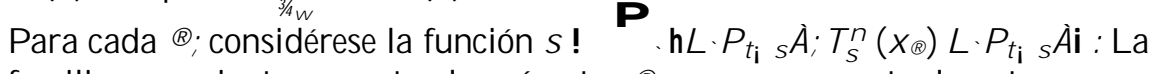
familia es creciente respecto al parámetro $Æ y$ converge puntualmente respecto a Æa la función continua $s ! \quad, h \cdot P_{t_{i}} s i ; T_{s}^{n}(x) L \cdot P_{t_{i}} s i i$ : A hora aplicandoel Lema de Dini $(1 ; 2 ; 10)$ a

$f_{A E}(s)=_{P} \cdot h \cdot P_{t_{i}} s i ; T_{s}^{n}\left(X_{F E}\right) L \cdot P_{t_{i}} s i i$ que es continua y convergente $a$

$f(s)=\cdot h \cdot P_{t_{i}} s i ; T_{s}^{n}\left(X_{E \in}\right) L \cdot P_{t_{i}}$ s $i$ esta convergencia es uniforme sobre

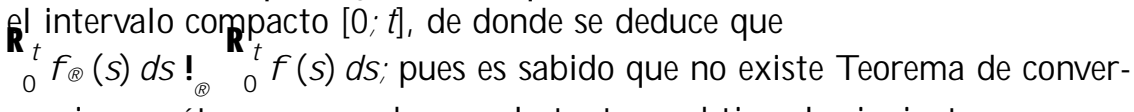
gencia monótona para redes, por lo tanto se obtiene lo siguiente:

$$
\begin{aligned}
& { }^{1} Z_{t} \\
& `=1 \quad 0 \\
& L \cdot P_{t_{i} s} u ; T_{s}^{n}(x) L \cdot P_{t_{i} s} \dot{i} d s
\end{aligned}
$$

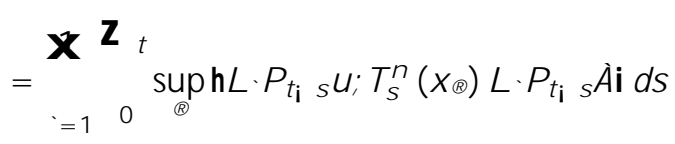

$$
\begin{aligned}
& ={ }_{=1}^{X} \sup _{\mathbb{E}}^{Z_{t}} h L \cdot P_{t_{i} s} u ; T_{s}^{n}\left(X_{F E} L \cdot P_{t_{i} s i} i d s\right. \\
& =\sup _{A E} x_{=1}^{Z_{t}} h \cdot P_{t_{i} s} u ; T_{s}^{n}\left(x_{A E}\right) L \cdot P_{t_{i}} s i i d s
\end{aligned}
$$

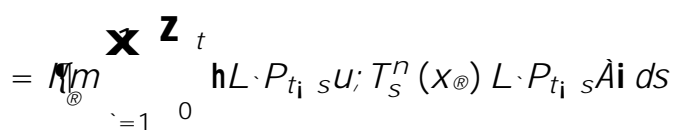

con lo cual

$$
\begin{aligned}
& \mathrm{u}^{-} \mathrm{T}_{\mathrm{t}}^{\mathrm{n}+1}(\mathrm{x}) \mathrm{u}^{\circledR}
\end{aligned}
$$

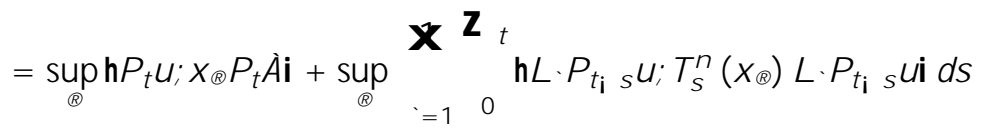

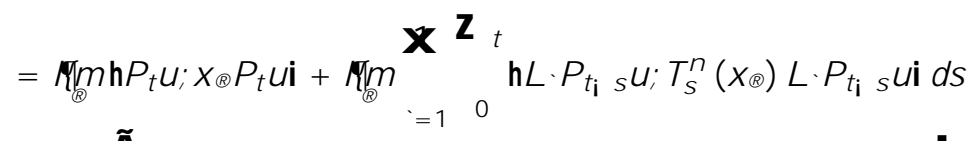

$$
\begin{aligned}
& \tilde{A} \\
& \times Z_{t}
\end{aligned}
$$

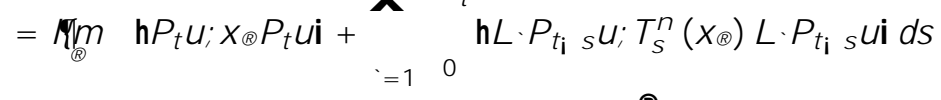

$$
\begin{aligned}
& =\lim { }_{\mathrm{E}}^{-} \mathrm{u} ; \mathrm{T}_{\mathrm{t}}^{\mathrm{n}+1}\left(\mathrm{x}_{\mathrm{FE}}\right) \mathrm{u}^{\circledR}
\end{aligned}
$$


resultando así que $T_{t}^{n+1}$ es normal.

De una forma similar se demuestra la Propiedad 2) para $T_{t}^{n+1}$. Para ello basta tomar $\Omega 2 \varnothing(h)$ y ver que $\operatorname{tr}\left(\Omega_{\mathrm{t}}^{\mathrm{n}+1}\right) \mathrm{t}_{\mathrm{t}} \operatorname{tr}\left(\Omega_{0}^{\mathrm{n}+1}\right)$ :

A hora veamos que $T_{t}^{n+1}$ es completamente positiva: Supongamos que $T_{t}^{n}$ es

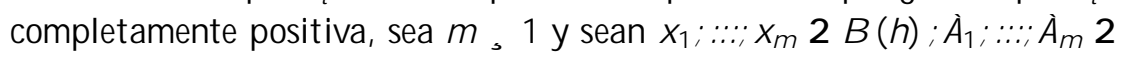
$D(G)$ entonces se tiene que

$$
\begin{aligned}
& x^{n}-i_{i} ; T_{t}^{n+1}\left(x_{i}^{\alpha} x_{j}\right) i_{j}{ }^{\circledR} \\
& i ; j=1 \\
& =x^{m} h P_{t} i_{i} ; x_{i}^{\alpha} x_{j} P_{t} i_{j} i \\
& i ; j=1
\end{aligned}
$$

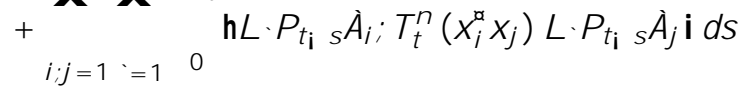

$$
\begin{aligned}
& =x^{n}-i_{i} ; T_{t}^{0}\left(x_{i}^{a} x_{j}\right) i_{j}^{\circledR} \\
& \begin{array}{lll}
i ; j=1 \\
X^{n} & x^{m} & Z_{t}
\end{array}
\end{aligned}
$$

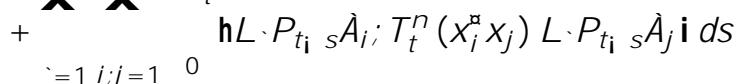

$$
\begin{aligned}
& x^{n}=1 \\
& =x^{m}-i_{i} ; T_{t}^{0}\left(x_{i}^{\not \alpha} x_{j}\right) i_{j}^{\circledR} \\
& x^{i ; j=1} Z_{t} x^{n}
\end{aligned}
$$

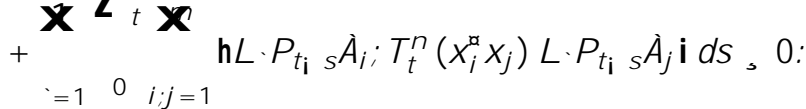

Esto demuestra que $T_{t}^{n+1}$ es completamente positivo.

Para la propiedad 4) supongamos que $T_{t}^{n}, T_{t}^{n_{i} 1}$, entonces

$$
\begin{aligned}
& -i{ }^{i} \mathrm{~T}_{\mathrm{t}}^{\mathrm{n}+1}(\mathrm{x}) \mathrm{i}_{\mathrm{t}}^{\mathrm{n}}(\mathrm{x})^{\phi}{ }^{\circledR}{ }^{\circledR}
\end{aligned}
$$

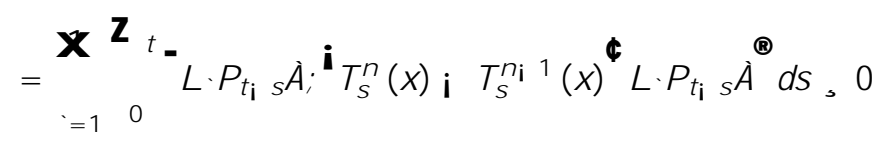

por lo tanto

$$
T_{t}^{n+1}(x), T_{t}^{n}(x):
$$

La propiedad 5); se demuestra así;

Tomemos u $2 \mathrm{D}(\mathrm{G})$ y supóngase como hipótesis de inducción que 
56CAPÍTULO 3. EL SEMIGRUPO DINÁMICO CUÁNTICO MINIMAL (S.D.C.M.)

$$
\begin{aligned}
& \mathrm{T}_{\mathrm{t}}^{\mathrm{n}}(1) \cdot 1: \\
& -\mathrm{u} ; \mathrm{T}_{\mathrm{t}}^{\mathrm{n}+1}(1) \mathrm{u}^{\circledR}=h \mathrm{P}_{\mathrm{t}} \mathrm{u} ; \mathrm{P}_{\mathrm{t}} \mathrm{ui}+\mathrm{X}^{\mathrm{B}} \mathrm{Z}_{\mathrm{t}} \\
& =1 Z^{0} \\
& L \cdot P_{t_{i} s} u ; T_{s}^{n}(1) L \cdot P_{t_{i}} s u i d s \\
& \text { - } \quad h P_{t} u ; P_{t} u i+\quad h L \cdot P_{t_{i} s} u ; L \cdot P_{t_{i} s} \text { ui ds } \\
& \mathrm{Z}_{\mathrm{t}}=1{ }^{0} \\
& =h P_{t} u ; P_{t} u i+{ }_{0} h \cdot P_{t_{i} s} u ; L \cdot P_{t_{i} s} u i d s \\
& \mathrm{Z}_{\mathrm{t}} \\
& \text { - hu; ui i } 2 \text { RehP }_{t_{i} s} u ; P_{t_{i} s} \text { Gui ds } \\
& =\text { hu;ui } i_{0}^{Z_{t}^{0}} \frac{d}{d s} k P_{t_{i} s} u k^{2} d s \\
& =h u ; u i \mathrm{i} k u k^{2}+k P_{t} u k^{2} \\
& =\text { hu; ui i hu; ui }+k P_{t} u k_{h}^{2} \\
& \text { - } k P_{t} k^{2} k u k^{2} \\
& \text {. hu; ui : }
\end{aligned}
$$

Por lo tanto

$$
\mathrm{T}_{\mathrm{t}}^{\mathrm{n}+1}(1) \cdot 1
$$

Esto termina la demostración.

EI siguiente Lema nos dice cómo construir el Semigrupo Dinámico M inimal, a partir de la proposición anterior. Pues aun cuando los $\mathrm{T}_{t}^{\mathrm{n}}$ no forman un semigrupo dinámico cuántico, a partir de estos el minimal resulta ser semigrupo dinámico cuántico y además resulta ser de contracciones. 
Lema 3.1.5 Supongamos la Hipótesis $A$. Entonces existe una familia $f T_{t} g_{t}, 0$ de transformaciones lineales contractivas sobre $B(h)$ tal que :

1) $T_{t}$ es completamente positiva para cada $t, 0$ :

2) $T_{t}$ es normal para cada $t, 0$ :

3) La familia de operadores lineales $f T_{t}(x) g_{t}, 0$ sobre $B(h)$ resuelve la ecuación $(3 ; 7)$ y $(3 ; 8)$ para todo $\times 2$ B (h) :

4) Para cada $x 2 B(h)$ la transformación $t$ ! $T_{t}(x)$ es continua con respecto a la topología æedébil en $B(h)$ :

Demostración. Seguimos [8], págs.49-50.

Fijemost, 0 y sea $f T_{t}^{n} g_{n}$, la sucesión de transformaciones lineales positivas como en la proposición anterior. A hora, para cada x 2 B (h) positivo, $0 \cdot T_{t}^{n}(x) \cdot j j T_{t}^{n}(x) j j_{1}: I \cdot j j x j j_{1}: l$; lo cual muestra que la sucesión fhu; $T_{t}^{n}(x)$ ui $g_{t, 0}$ de números reales positivos, es creciente y acotada, es decir hu; $T_{t}^{n}(x)$ ui · jjxjj $j_{1} j j_{j} j_{h}^{2}$ : Así denotamos ahora el $\lim _{n !} h u ; T_{t}^{n}(x)$ ui $=u ; T_{t}^{m i n}(x) u$, para cada $u 2$ h: Para el caso general sólo usamos la Identidad de Polarización que dice lo siguiente:

$$
4 h u ; T_{t}(x) i i=x_{k=0}^{X^{3}} i k^{-} u+i^{k} i ; T_{t}(x) i+i^{k}{ }_{i} \stackrel{d \circledR}{:}
$$

para cada u; ¿ 2 h: Y para $\times 2$ B (h) arbitrario, sólo lo escribimos como combinación lineal de 4 operadores positivos.

Claramente cada $T_{t}^{m i n}$ es una contracción pues $T_{t}^{n}$ lo es al igual que goza de la propiedad 1.

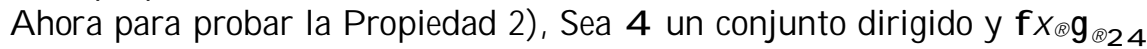
una red de operadores positivos en $B(h)$ con supremo $x$; ya que las transformaciones $T_{t}^{n}(n, 0)$ son æedébil continuas, para cada $i 2 \mathrm{~h}$ se tiene que

$$
\begin{aligned}
& \operatorname{Suph}_{\mathbb{E}} ; \mathrm{T}_{\mathrm{t}}\left(\mathrm{x}_{\mathbb{E}}\right) \dot{\mathrm{i}}
\end{aligned}
$$

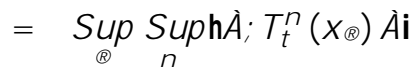

$$
\begin{aligned}
& =\operatorname{Sup}_{\mathrm{n}} \operatorname{Suph}_{\mathbb{E}} ; T_{\mathrm{t}}^{\mathrm{n}}\left(\mathrm{x}_{\mathbb{E}}\right) i^{\mathrm{i}} \\
& =\operatorname{Suph}_{\mathrm{n}} ; T_{\mathrm{t}}^{\mathrm{n}}(\mathrm{x}) \mathrm{i}^{\mathrm{i}} \\
& =h_{i} ; T_{t}(x) i^{i}
\end{aligned}
$$

lo cual demuestra que $T_{t}^{\text {min }}=T_{t}$ es normal. En la Ecuación $(3 ; 8)$; si hacemos $n$ ! 1 se obtiene la E cuación $(3 ; 7)$ y a la vez la Ecuación $(3 ; 6)$, pues ambas son equivalentes.

Finalmente para cada u; $i 2$ h y x 2 B (h) la función $t$ ! h; $T_{t}(x)$ ¿i es continua por la Ecuación (2;5), pero como $k T_{t}(x) k_{1} \cdot k x k_{1}$; entonces resulta uniformemente acotado por lo que la continuidad se da tanto en la topología débil como en la æedébil. 


\section{CAPÍTULO 3. EL SEMIGRUPO DINÁMICO CUÁNTICO MINIMAL (S.D.C.M.)}

Proposición 3.1.6 Consideremos $\left(\mathrm{W}_{t}^{\mathrm{n}}\right)_{\mathrm{n}}$ o la sucesión de transformaciones lineales positivas en $B(h)$ de.nidas inductivamente por

$$
\begin{aligned}
\mathrm{W}_{\mathrm{t}}^{0} & =\mathrm{T}_{\mathrm{t}}^{0} \\
\mathrm{~W}_{\mathrm{t}}^{\mathrm{n}+1} & =\mathrm{T}_{\mathrm{t}}^{\mathrm{n}+1} \mathrm{i} \mathrm{T}_{\mathrm{t}}^{\mathrm{n}}
\end{aligned}
$$

entonces para cada $\mathrm{n}, 0$ se tiene la siguiente identidad:

$$
w_{t+s}^{n+1}(x)=x_{k=0}^{n} w_{t}^{k} W_{s}^{n_{i} k}(x)^{\phi}
$$

Demostración. Seguimos [8], págs.51-52.

Claramente de la ecuación $(3 ; 8)$ se tiene que

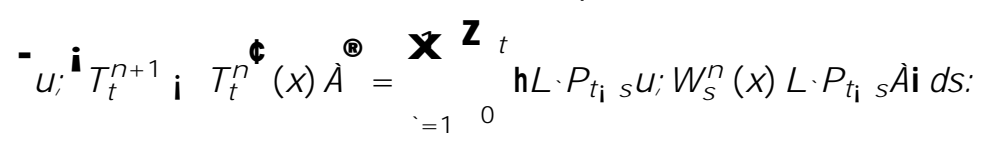

Entonces,

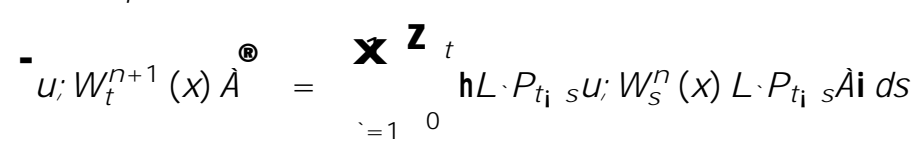

para cada u; ¿ $2 \mathrm{D}(\mathrm{G})$; $\times 2 \mathrm{~B}(\mathrm{~h})$ y $\mathrm{n}, 0$ :

Para $\mathrm{n}=0$ no hay nada que mostrar pues

$$
\begin{gathered}
\mathrm{W}_{\mathrm{t}+\mathrm{s}}^{0}(\mathrm{x})=\mathrm{T}_{\mathrm{t}+\mathrm{s}}^{0}=\mathrm{T}_{\mathrm{t}}^{0}{ }^{\mathrm{i}} \mathrm{T}_{\mathrm{s}}^{0}(\mathrm{x})^{\phi} \\
=\mathrm{W}_{\mathrm{t}}^{0}{ }^{\mathrm{i}} \mathrm{W}_{\mathrm{s}}^{0}(\mathrm{x})^{\phi}:
\end{gathered}
$$

A sí que supongamos que la ecuación $(3 ; 9)$ se cumple para algún $\mathrm{n} 2 \mathrm{~N}$ : U sando (3;9;) para cada u; $i 2 \mathrm{D}(\mathrm{G})$ y $\times 2$ B (h) se tiene lo siguiente

$$
\begin{aligned}
& { }^{x+1}-w_{t} W_{t}^{k} W_{s}^{n+1} 1_{i}(x)^{\phi} i^{\circledR} \\
& k=0
\end{aligned}
$$

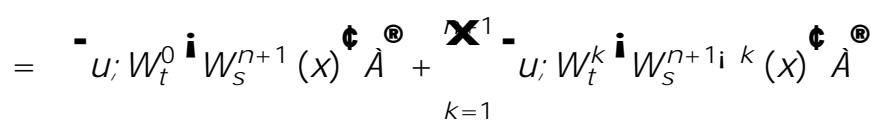




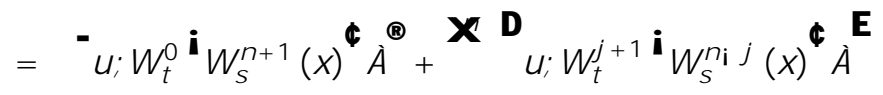

$$
\begin{aligned}
& =-{ }^{-} ; w_{t}^{0}{ }^{i} W_{s}^{n+1}(x)^{\phi} i^{\circledR}{ }^{j=0} \\
& +X^{n} x^{A} Z_{t_{-}} L \cdot P_{t_{i} r} u ; W_{r}^{j}{ }^{i} W_{s}^{n_{i} j}(x)^{\Phi} L \cdot P_{t_{i} r i}{ }^{\circledR} d r \\
& j=0=\begin{array}{lll} 
& 0
\end{array} \\
& ={ }^{-} \mathrm{u} ; \mathrm{w}_{\mathrm{t}}{ }^{0} \mathrm{i}_{\mathrm{s}}^{\mathrm{n}+1}(\mathrm{x})^{\Phi}{ }^{\circledR}{ }^{\circledR} \\
& +X^{A} X^{n} Z_{t_{-}} L \cdot P_{t_{i} r} u ; W_{r}^{j}{ }^{i} W_{s}^{n_{i} j}(x){ }^{\Phi} L \cdot P_{t_{i} r i}{ }^{\circledR} d r \\
& ={ }^{-} u_{i} ; w_{t}{ }^{0}{ }^{i} W_{s}^{n+1}(x){ }^{\Phi}{ }^{\circledR}
\end{aligned}
$$

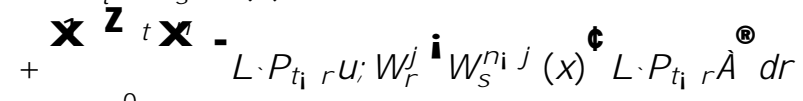

$$
\begin{aligned}
& =\begin{array}{c}
-u_{i} W_{t}^{0}{ }^{i} W_{* s}^{j=0}{ }^{n+1}(x)^{\Phi}{ }_{i}^{\circledR} \\
x^{\circledR} Z_{t} X^{n}
\end{array}+ \\
& +\quad L \cdot P_{t_{i} r} u_{i}{ }^{n} W_{r}^{j}{ }^{i} W_{s}^{n_{i j} j}(x)^{\Phi} L \cdot P_{t_{i} r i} d r \\
& =-{ }^{`}=1 \mathrm{~W}_{\mathrm{t}}^{0}{ }^{\mathrm{i}} \mathrm{W}_{\mathrm{s}}^{\mathrm{n}+1}(\mathrm{x})^{\phi} i^{\mathrm{j}{ }^{\mathrm{B}}=0} \\
& +{ }_{=1}^{X}{ }^{X} Z_{t_{-}} L \cdot P_{t_{i} r} u ; W_{r+s}^{n}(x) L \cdot P_{t_{i} r} i \stackrel{\circledR}{d r} \text {; } \\
& \text { de donde obtenemos que } \\
& \mathrm{x}^{+1}-\mathrm{u}_{\mathrm{k}=0} \mathrm{~W}_{\mathrm{t}}^{\mathrm{k}}{ }^{\mathrm{i}} \mathrm{W}_{\mathrm{s}}^{\mathrm{n}+1_{\mathrm{i}} \mathrm{k}}(\mathrm{x}){ }^{\Phi}{ }_{i}^{\circledR}
\end{aligned}
$$

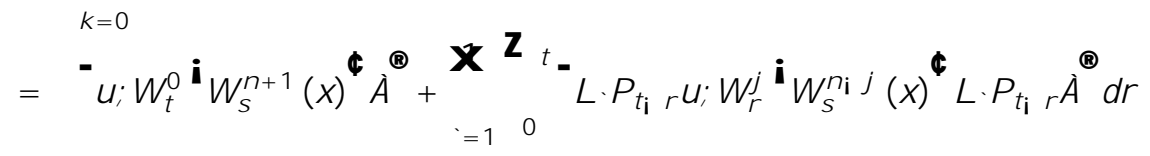

$$
\begin{aligned}
& \text { pero volviendo a la de..nición de } \mathrm{W}_{\mathrm{t}}^{0} \text {; el primer sumando de esta igual- } \\
& \text { dad es igual a } \\
& \mathrm{w}_{\mathrm{t}}^{0 \mathrm{i}} \mathrm{W}_{\mathrm{s}}^{\mathrm{n}+1}(\mathrm{x})^{\phi} \\
& =T_{t}^{0}{ }^{i} W_{s}^{n+1}(x)^{\phi}=P_{t}^{x}{ }^{i} W_{s}^{n+1}(x)^{\Phi} P_{t} \text {; }
\end{aligned}
$$


60CAPÍTULO 3. EL SEMIGRUPO DINÁMICO CUÁNTICO MINIMAL (S.D.C.M.)

por lo tanto,

$$
\begin{aligned}
& \mathrm{x}^{+1}-\mathrm{u} ; \mathrm{W}_{\mathrm{t}}^{\mathrm{k}}{ }^{\mathrm{i}} \mathrm{W}_{\mathrm{s}}^{\mathrm{n}+1_{\mathrm{i}} \mathrm{k}}(\mathrm{x})^{\Phi} i^{\circledR}
\end{aligned}
$$

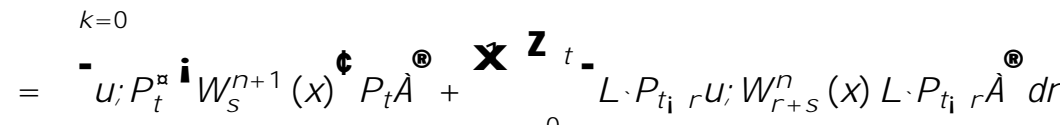

$$
\begin{aligned}
& =-P_{t} u ; W_{s}^{n+1}(x) P_{t}{ }^{\circledR}+{ }^{x}{ }^{i \bar{Z}_{t}^{1}}{ }^{0} L \cdot P_{t_{i} r} u ; W_{r+s}^{n}(x) L \cdot P_{t_{i} r i}{ }^{\circledR} d r \\
& ={ }^{-} P_{t} u ; W_{s}^{n+1}(x) P_{t} i^{\circledR}+{ }^{i=1} Z_{t+s}^{0} h \cdot P_{t+s} r u ; W_{r}^{n}(x) L \cdot P_{t+s i r} \dot{i} d r \\
& =-P_{t} u ; W_{s}^{n+1}(x) P_{t} i^{\circledR}{ }_{=1}^{i=1} Z_{s}^{s} h \cdot P_{s i r} P_{t} u ; W_{r}^{n}(x) L \cdot P_{s i r} P_{t} i i d r \\
& +X_{t+s}^{Z^{*}} L \cdot P_{t+s i r} u ; W_{r}^{n}(x) L \cdot P_{t+s i} r i i d r \\
& =x_{=1}^{i=\frac{1}{Z}{ }_{t+s}^{0}} h \cdot P_{t+s i} r u ; W_{r}^{n}(x) L \cdot P_{t+s i} r i i d r \\
& =-\mathrm{u}_{;} \mathrm{W}_{\mathrm{t}+\mathrm{s}}^{\mathrm{n}+1}(\mathrm{x}) \dot{i}^{\circledR}:
\end{aligned}
$$

Así se tiene que,

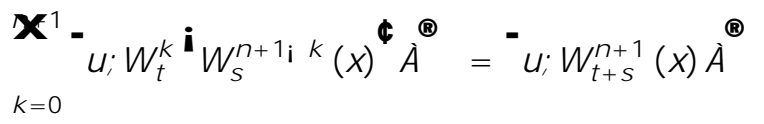

$$
\begin{aligned}
& u_{k=0}^{*+1} W_{t}^{k}{ }^{i} W_{s}^{n+1 i k}(x)^{\phi} i^{+}=-u ; W_{t+s}^{n+1}(x) i^{\circledR} \\
& W_{t+s}^{n+1}(x)={ }_{k=0}^{x+1} W_{t}^{k} W_{s}^{n+1} k^{i}(x)^{\phi}
\end{aligned}
$$

lo que termina la prueba. 


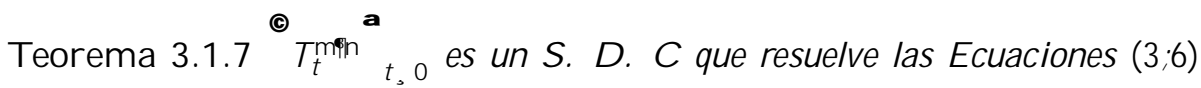
y $(3 ; 7)$ con la siguiente propiedad: Para cualquier otra familia ædébil continua $\mathrm{fT}_{\mathrm{t}} \mathrm{g}_{\mathrm{t}, 0}$ de transformaciones lineales positivas en $\mathrm{B}(\mathrm{h})$ que satisfaga las ecuaciones $(3 ; 6),(3 ; 7)$ y $\times 2 \mathrm{~B}(\mathrm{~h})$ positivo, se tiene

$$
T_{t}^{\min }(x) \cdot T_{t}(x)
$$

para todo $\mathrm{t}, 0$ :

Demostración. Seguimos [8], pág.51.

Demostraremos que este es un Semigrupo Dinámico Cuántico, es decir

que satisface la propiedad

$$
\mathrm{T}_{\mathrm{t}+\mathrm{s}}^{\min }(\mathrm{x})=\mathrm{T}_{\mathrm{t}}^{\mathrm{min}} \mathrm{i}^{\mathrm{i}} \mathrm{T}_{\mathrm{s}}^{\mathrm{min}}(\mathrm{x})^{\phi}
$$

para $\times 2$ B (h) postivo y s; $t$,

Por el lema anterior, tenemos la siguientes identidades

$$
\begin{aligned}
& -i ; T_{\mathrm{t}+\mathrm{s}}^{\mathrm{n}}(\mathrm{x}) i^{\circledR} \\
& =x_{k=0}^{n}-i ; W_{t+s}^{k}(x) i^{\circledR}
\end{aligned}
$$

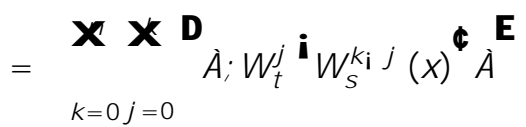

$$
\begin{aligned}
& =\sum_{j=0 k=j}^{n} X^{n} D_{i} ; W_{t}^{j}{ }^{i} W_{s}^{k_{i} j}(x)^{\phi}{ }_{i}^{E}
\end{aligned}
$$

para cada ¿ 2 h; y $\times 2$ B (h): Es decir

$$
-i ; T_{t+s}^{n}(x) i \stackrel{\circledR}{=} x_{j=0}^{n} D ; ; W_{t}^{j}{ }^{i} T_{s}^{n_{i} j}(x)^{\phi}{ }_{i}^{E}
$$




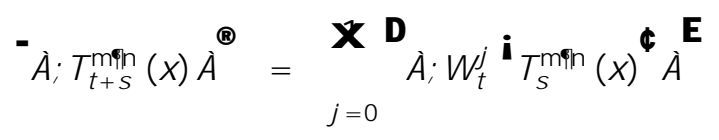

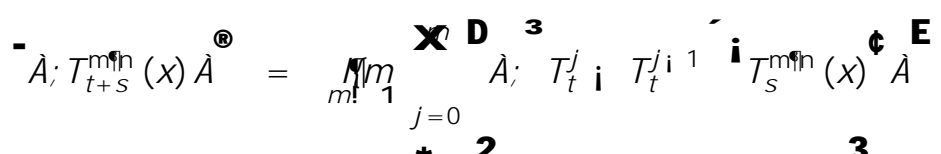

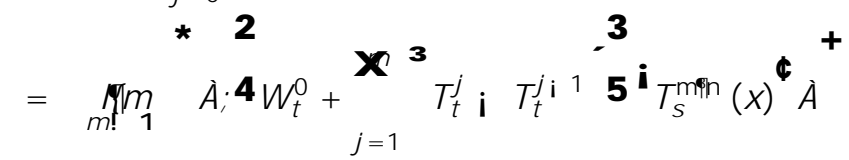

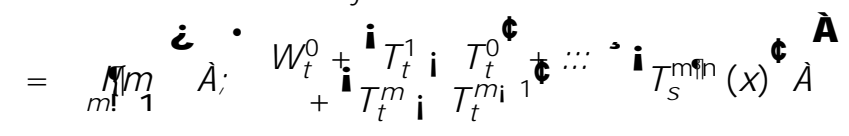

$$
\begin{aligned}
& =\lim _{m ! 1}-i ; T_{t}^{m}{ }^{i} T_{s}^{m m n}(x)^{\Phi}{ }^{\circledR}
\end{aligned}
$$

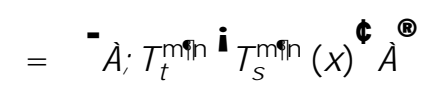

así se tiene que una vez más por la identidad de Polarización, y descomponiendo a $x$ como suma de 4 operadores positivos

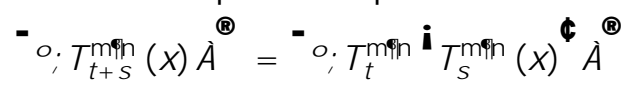

por lo tanto

$$
\mathrm{T}_{\mathrm{t}+\mathrm{s}}^{\min }(\mathrm{x})=\mathrm{T}_{\mathrm{t}}^{\min } \mathrm{i}^{\mathrm{m}} \mathrm{s}_{\mathrm{s}}^{\min }(\mathrm{x})^{\phi}:
$$

Pero por la parte (4) de la Proposición (3;1;4) se tiene también lo siguiente

$$
\mathrm{T}_{\mathrm{t}}^{\mathrm{m} \text { fin }}(1) \cdot 1 ;
$$

y de la parte (2) de ésta misma Proposición (3;1;4), es claro que

$$
T_{t}(x), P_{t}^{\infty} x P_{t}=T_{t}^{0}(x):
$$

Si suponemos $T_{t}(x), T_{t}^{n}(x)$ para algún $n 2 N$, entonces para cada $i 2 h$ se tiene que

$$
\begin{aligned}
& { }^{-}{ }^{i}{ }^{\mathrm{i}} \mathrm{T}_{\mathrm{t}}(\mathrm{x}) \mathrm{i}_{\mathrm{t}}^{\mathrm{n}+1}(\mathrm{x})^{\Phi}{ }^{\circledR}{ }^{\circledR} \\
& { }^{2} \mathrm{Z}_{\mathrm{t}}
\end{aligned}
$$

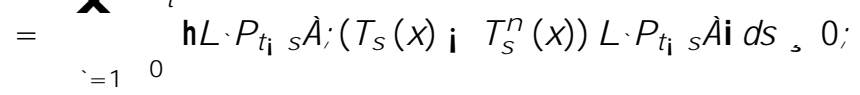

esto muestra que

$$
T_{t}^{n+1}(x) \cdot T_{t}(x)
$$


con lo cual haciendo $n ! 1$ se tiene que $T_{t}^{m m}(x) \cdot T_{t}(x)$ para $t, 0$.

El siguiente corolario nos revela que la propiedad de conservatividad es una condición su..ciente, para la unicidad de la solución de la ecuación de Lindblad, es decir la Ecuación (3;7) ; para ello recordemos que un semigrupo dinámico cuánticof $T_{t} g_{t}, 0$ es conservativo si $T_{t}(I)=I$ :

Corolario 3.1.8 Supóngase que ${ }^{\complement} \mathrm{T}_{\mathrm{t}}^{\text {mm }}{ }^{\text {a }} \mathrm{t}_{0}$ es conservativo. Entonces es la única familia $\mathrm{fT}_{\mathrm{t}} \mathrm{g}_{\mathrm{t}, 0}$ de transformaciones lineales, ædébil continuas, positivas y contractivas sobre $B(h)$ que satisface la ecuación $(3 ; 6)$.

Demostración. Seguimos [8], pág.53.

Sea $T=f T_{t} g_{t}$ o una familia de transformaciones lineales positivas, æéébil continuas que satisface la Ecuación $(3 ; 6)$ : A plicando el Teorema anterior para cada $\times 2 \mathrm{~B}(\mathrm{~h})$ tal que $0 \cdot \mathrm{x} \cdot 1 \mathrm{y}$ cada $\mathrm{t}, 0$ tenemos

$$
\begin{aligned}
& T_{t}^{m i n}(x) \cdot T_{t}(x) \\
& =T_{t}(1) ; T_{t}(1 ; x) \\
& \text { - } 1 ; \mathrm{T}_{\mathrm{t}}(1 ; \mathrm{x}) \\
& \text { - } \quad 1 ; T_{t}^{m m n}(1 ; x) \\
& =1 \mathrm{i} \mathrm{T}_{\mathrm{t}}^{\mathrm{mmn}}(1)+\mathrm{T}_{\mathrm{t}}^{\mathrm{mmn}}(\mathrm{x})=\mathrm{T}_{\mathrm{t}}^{\mathrm{m} \text { mn }}(\mathrm{x}) \text { : }
\end{aligned}
$$

Por lo tanto $T_{t}^{m f i n}(x)=T_{t}(x)$ para todo $x, 0$ : Para $x 2$ B (h) arbitrario lo expresamos como combinación lineal de 4 operadores positivos.

\subsection{EI Resolvente del Semigrupo M inimal}

En la sección anterior se construyó el semigrupo dinámico cuántico minimal, asociado a los operadores $G$ y $L$ - que satisfacen la H ipótesis A. Como se habrá observado este semigrupo satisface las ecuaciones $(3 ; 6)$ y $(3 ; 7)$, pero en general este semigrupo no está caracterizado por tal propiedad, nos gustaría conocer el dominio del generador in..nitesimal de $T^{\mathrm{m} \text { min }}$, cosa que no resulta nada fácil conociendo simplemente a los operadores $G$ y $L$.

Proposición 3.2.1 Supóngase que se tiene la Hipótesis $A$. Las transformaciones lineales positivas $F_{\Pi}: B(h) ! B(h)$ y $\quad Q_{\Pi}: B(h) ! B(h)$ de..nidas por

$$
\begin{aligned}
& h \int ; F_{\Pi}(x) i i=Z_{1} e^{i{ }^{s} h P_{s} \int ; x P_{s} i i d s} \\
& h f ; Q_{\Pi}(x) i i=x_{=0}^{Z_{1}} e^{i \Pi^{s} h L \cdot P_{s} \int ; x L \cdot P_{s} i i d s}
\end{aligned}
$$


para $\Pi>0 ; \times 2 \mathrm{~B}(\mathrm{~h}), \int ;$ i $2 \mathrm{D}(\mathrm{G})$ son normales y completamente positivas. Además $\mathrm{kF}_{\Pi} \mathrm{k}_{\mathrm{B}(\mathrm{B}(\mathrm{h}))} \cdot \Pi^{1}{ }^{1}, \mathrm{kQ}_{\Pi} \mathrm{k}_{\mathrm{B}(\mathrm{B}(\mathrm{h}))} \cdot 1$ :

Demostración. Ver. [8] Págs. 54 i 56; [5] Pág.104.

De..nición 3.2.2 Se de.ne el resolvente minimal ${ }^{i} R_{\Pi}^{\min }{ }_{\Pi>0}^{\phi}$ del semigrupo dinámico cuántico minimal(SDCM) por medio de su forma sesquilineal:

$$
\int_{i} R_{\Pi}^{\min }(x) i^{\circledR}={ }_{0}^{Z_{1}} e^{i \Pi s^{-}} \int ; T_{s}^{\text {min }}(x) i^{\circledR} d s
$$

$\operatorname{con} \times 2$ B (h) y $\quad$ i; $2 \mathrm{~h}$ :

Teorema 3.2.3 Para cada $\Pi>0$ y $\times 2$ B (h) se tiene lo siguiente:

$$
\mathrm{R}_{\Pi}^{\min }(\mathrm{x})={ }_{\mathrm{k}=0}^{\mathrm{X}} \mathrm{Q}_{\Pi}^{\mathrm{k}}\left(\mathrm{F}_{\Pi}(\mathrm{x})\right)
$$

donde la serie converge en la topología fuerte de operadores.

Demostración. Seguimos [8], pág.55.

Sea $\left(R_{\Pi}^{n}\right)_{n, 0}$ la sucesión de tranformaciones lineales completamente positivas $\mathrm{R}_{\Pi}^{\mathrm{n}}: \mathrm{B}(\mathrm{h}) ! \mathrm{B}(\mathrm{h})$ dadas por

$$
\mathrm{h} f ; \mathrm{R}_{\Pi}^{\mathrm{n}}(\mathrm{x}) \dot{i}={ }_{0}^{\mathrm{Z}_{1}} \mathrm{e}^{\mathrm{i} \Pi \mathrm{s}} \mathrm{h} f ; \mathrm{T}_{\mathrm{s}}^{\mathrm{n}}(\mathrm{x}) \dot{i} \mathrm{ds}
$$

donde los $\mathrm{T}_{\mathrm{s}}^{\mathrm{n}}$ están de..nidos por la E cuación (3;8), Proposición 3;1;4:

Ya que las transformaciones $T_{s}^{n}$ son contracciones las $R_{\Pi}^{n}$ están bien de..nidas, además para cada $0 \cdot \times 2$ B (h);

$$
\begin{array}{ll}
= & Z_{1} ; R_{\Pi}^{n}(x) i i \\
= & Z^{i}{ }^{i s} h u ; T_{s}^{n}(x) i i d s \\
= & e^{i} e^{-} u ; T_{s}^{n+1}(x) i{ }^{\circledR} d s \\
= & u ; R_{\Pi}^{n+1}(x) i^{\circledR}:
\end{array}
$$

A sí para cada $\Pi>0$ y $0 \cdot x 2$ B (h), $f R_{\Pi}^{n}(x) g_{\Pi, 0}$ es no decreciente. Por lo tanto por la de..nición del S. D. C. M.,, para todo $i 2 \mathrm{~h}$ y todo $0 \cdot \times 2$ B (h) se tiene lo siguiente,

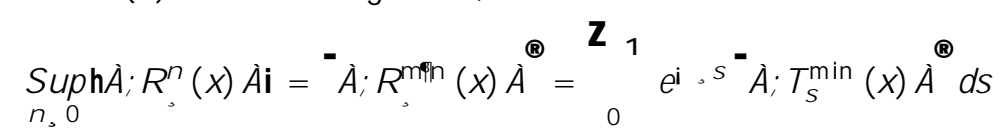


y otra vez por la Ecuación $(3 ; 8)$ se tiene que

$$
\begin{aligned}
& { }_{i ; R_{\Pi}^{n+1}(x) i}{ }^{\circledR}={ }^{Z_{1}} e^{i \pi t} h P_{t} i ; x P_{t i} i d t+ \\
& x_{=1}^{x^{0} Z_{1}} e^{Z^{i t} d t}{ }_{0}^{Z_{t}} L \cdot P_{t_{i}} s i ; T_{s}^{n}(x) L \cdot P_{t_{i}} s i i d s
\end{aligned}
$$

para todo ¿; 2 $2 \mathrm{D}(\mathrm{G})$ :

A hora con el cambio de variable $(r ; s)=(t ; s ; s)$; en la integral doble de arriba, se tiene que la suma de los terminos dela derecha es igual a

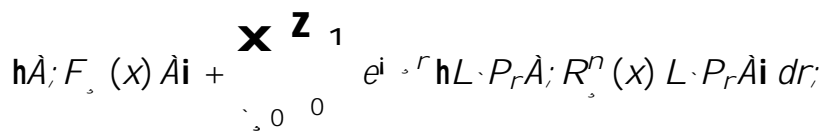

es decir, tenemos la siguiente fórmula recursiva

$$
R_{\Pi}^{n+1}(x)=F_{\Pi}(x)+Q_{\Pi}\left(R_{\Pi}^{n}(x)\right)
$$

que, al iterarla $n$ veces nos da,

$$
R_{\Pi}^{n+1}(x)={ }_{k=0}^{x+1} Q_{\Pi}^{k}\left(F_{\Pi}(x)\right):
$$

Al hacer $n ! 1$, se tiene que $R_{\Pi}^{m i n}(x)=P_{k=0}^{P_{n}} Q_{\Pi}^{k}\left(F_{\Pi}(x)\right)$ : Para generalizar esto a todo $\times 2$ B (h) ; recordemos que $x$ se expresa como combinación lineal de 4 operadores positivos y acotados.

A hora consideramos el predual de nuestro S. D. C. M . EI Teorema 1;2;11 del Capítulo 1 nos garantiza que tal semigrupo existe y lo denotaremos por $S^{m}$, éste actúa sobre el espacio de los operadores de traza fínita $i$ (h) (Ver. [3] Capítulo3): Otra forma de caracterizar la conservatidad del semigrupo dinámico cuántico minimal es que su predual conserve a la traza, como lo muestra la siguiente proposición.

P roposición 3.2.4 Supongamos la Hipótesis A. Entonces para cada t, 0 las siguientes condiciones son equivalentes:

1) $T_{t}^{m m n}(I)=I_{\phi} ;$ para todo $t, 0$

2) $\operatorname{tr}^{i} S_{t}^{\text {min }}(\Omega)=\operatorname{tr}(\Omega$ para cada $\Omega 2 \varnothing($ h) ;y todo $t, 0$ :

Demostración. Seguimos [8], pág.56.

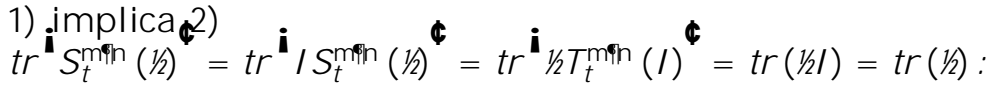


66CAPÍTULO 3. EL SEMIGRUPO DINÁMICO CUÁNTICO MINIMAL (S.D.C.M.)

2) implica 1)

Supongamos que

$$
\operatorname{tr}^{\mathrm{i}} \mathrm{S}_{\mathrm{t}}^{\min }\left(\Omega^{\Phi}=\operatorname{tr}(\Omega) \text { : para cada } \Omega 2 \varnothing(\mathrm{h}) \text { y todo } \mathrm{t}, 0\right. \text {; }
$$

entonces

$$
\operatorname{tr}^{i} \Omega_{t}^{m m^{m}}(I){ }^{\phi}=\operatorname{tr}(\Omega) \text {; }
$$

por lo tanto

$$
\operatorname{tr}^{\mathrm{i}} \Omega^{\mathrm{i}} \mathrm{T}^{\min }(\mathrm{I}) ; \mathrm{I}^{\not \subset}=0
$$

para todo $\Omega 2 \varnothing(\mathrm{h})$. Pero el único elemento de $B(\mathrm{~h})$ que tiene esta propiedad es el operador 0 ; lo que prueba que $T_{t}^{\min }(I)=I$ :

Sean u; ¿ $2 D(G)$; si $T_{t}^{\min }$ es conservativo, es decir $T_{t}^{\min }(I)=1$; para todo $t$, 0 entonces se tiene que

$$
\begin{aligned}
& \lim _{\mathrm{t} !} \frac{\mathrm{T}_{0^{+}}^{\min }(\mathrm{I}) \mathrm{i} \mid}{\mathrm{t}}=0 \text {; } \\
& \text { entonces I } 2 D^{i} \$^{\min }{ }^{\phi} y \\
& \$^{m i n}(1)=\lim _{t !} \prod_{0_{0^{+}}} \frac{T_{t}^{\min }(1) i \mid}{t}
\end{aligned}
$$

por lo tanto se tiene así que

$$
-\int^{\min }(1) i^{\circledR}=0
$$

pero otra parte también se tiene

$$
\text { hu; } \$(1) i i=-u ; \$^{m m}(1) i^{\circledR}
$$

por lo tanto

$$
h u ; \$(I) i i=h G u ; i i+h u ; G_{i} i+{ }_{=1}^{X} h L \cdot u ; L \cdot i i=0
$$

que es la ecuación de la parte ii) en la Hipótesis $A$ A, que a continuación se presenta.

Hipótesis AA.

i) Sea $G$ el generador in..nitesimal de un semigrupo de contracciones fuertemente continuos $f P_{t} g_{t, 0}$ en $h$ : 
ii) Sea $f L \cdot g^{1}=1$ operadores no necesariamente acotados de tal forma que

$$
D(G){ }_{`=1}^{1 / 2} D(L \cdot) ;
$$

y para cada u 2 D (G)

$$
h u ; G u i+h G u ; u i+{ }_{=1}^{X} h \cdot u ; L \cdot u i=0:
$$

Hay que observar que hemos convertido en igualdad a la desigualdad de la parte ii) de la Hipótesis A. Esta igualdad es una condición necesaria, aunque no su..ciente para que el semigrupo minimal sea conservativo. Para estudiar condiciones equivalentes a la conservatividad empezamos por demostrar la siguiente proposición.

Proposición 3.2.5 Supongamos que se tiene la Hipótesis A A y ..jemos $\Pi>$ $0:$ Para todo $n, 1$ se tiene lo siguiente:

$$
\prod_{k=0}^{X n} Q_{\Pi}^{k}\left(F_{\Pi}(1)\right)+Q_{\Pi}^{n+1}(1)=1 \text { : }
$$

Demostración.

Seguimos [8], pág.57.

Para cada $i 2$ D (G), Pt 2 D (G) : Por lo que usando la Hipótesis A A se tiene

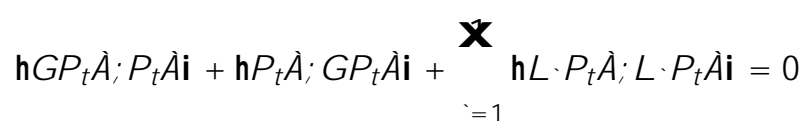

y

$x$

$h \cdot P_{t i} ; L \cdot P_{t} i i=i \quad\left[h G P_{t} i ; P_{t} i i+h P_{t i} ; G P_{t} i\right]=i 2 R$ ehP $P_{t i} ; G P_{t i} i$ $=1$

al multiplicar por ei $\Pi^{\mathrm{t}}$ e integrar ,

$$
\begin{aligned}
& Z_{1} \\
& h_{i} ; Q_{\Pi}(1) i i={ }_{0} Z_{1} e^{i t}\left(i 2 R \operatorname{ehP} t i ; G P_{t} i i\right) d t \\
& =i_{0}^{1} e^{i} \frac{d}{d t} k P_{t} i k_{h}^{2} d t \\
& =k_{i} k_{h}^{2} i \Pi_{0} e^{i \Pi^{t} k P_{t} i k_{h}^{2} d t} \\
& =k_{i} k_{h}^{2} i \prod_{i} ; F_{\Pi}(1) i i \text {; }
\end{aligned}
$$


por lo tanto

$$
\Pi_{\Pi}(1)+Q_{\Pi}(1)=1:
$$

Supongamos que (3;13) se cumple para algún n $2 \mathrm{~N}$; al aplicar $\mathrm{Q}_{\Pi}$ a la hipótesis de inducción obtenemos,

$$
\begin{aligned}
& \mathrm{Q}_{\Pi}^{\tilde{\mathrm{A}}} \prod_{\mathrm{k}=0}^{\mathrm{X}} \mathrm{Q}_{\Pi}^{\mathrm{k}}\left(\mathrm{F}_{\Pi}(1)\right)+\mathrm{Q}_{\Pi}^{\mathrm{n}+1}(1)=\mathrm{Q}_{\Pi}(1) \\
& \prod_{k=0}^{X} Q_{\Pi}^{k+1}\left(F_{\Pi}(1)\right)+Q_{\Pi}^{n+2}(1)=1 i \Gamma_{\Pi}(1) \\
& \Pi_{\Pi}\left(F_{\Pi}(1)\right)+:: .+\Pi_{\Pi}^{n+1}\left(F_{\Pi}(1)\right)+Q_{\Pi}^{n+2}(1)=1 ; \Pi_{\Pi}(1)
\end{aligned}
$$

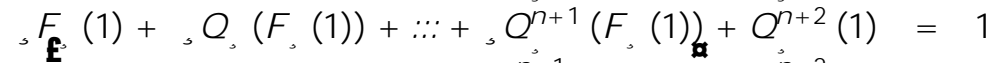

$$
\begin{aligned}
& \Pi F_{\Pi}(1)+Q_{\Pi}\left(F_{\Pi}(1)\right)+:: \cdot+Q_{\Pi}^{n+1}\left(F_{\Pi}(1)\right)+Q_{\Pi}^{n+2}(1)=1 \\
& \prod_{k=0}^{X+1} Q_{\Pi}^{k}\left(F_{\Pi}(1)\right)+Q_{\Pi}^{n+2}(1)=1:
\end{aligned}
$$

Por lo tanto, $(3 ; 14)$ vale para todo $\mathrm{n}$ entero no negativo.

La representación del resolvente minimal por medio de la ecuación (3;13); ahora nos permite probar una condición necesaria y su..ciente para la conservatividad, debida a A. M. Chebotarev y a F. Fagnola.

Teorema 3.2.6 Supongamos que tenemos la Hipótesis AA y sea $\Pi>0$..jo. Las siguientes condiciones son equivalentes:

1) $T_{t}^{m \text { min }}$ es conservativo, para todo $t, 0$;

2) $\lim _{n !} Q_{\Pi}^{n}(1)=0$;

3) EI único elemento $x 2 \mathrm{~B}(\mathrm{~h})$ que cumple con la ecuación $\mathrm{Q}_{\Pi}(\mathrm{x})=\mathrm{x}$ es $x=0$ :

Demostración.

Seguimos [8], pág.58.

1) implica 2)

Como $T_{t}^{m m}(1)=1$; de la de.nición de $R_{\Pi}^{\min }$ (1) se deduce que:

$$
{ }^{-} u_{;} R_{\Pi}^{\min }(1) u^{\circledR}={ }_{0}^{Z_{1}} e^{i t^{-}} u ; T_{t}^{m m n}(1) u^{\circledR} d t=\frac{k u k_{h}^{2}}{\Pi}=u^{D} ; \frac{u^{E}}{\Pi} ;
$$

por lo tanto

$$
\mathrm{R}_{\Pi}^{\mathrm{mm}}(1)=\frac{1}{\Pi}:
$$


Pero en vista de la ecuación $(3 ; 11)$; también se tiene que

$$
\prod_{\mathrm{k}=0}^{\mathrm{X}} \mathrm{Q}_{\Pi}^{\mathrm{k}}\left(\mathrm{F}_{\Pi}(1)\right)=1 ;
$$

por lo tanto en la ecuación (3;13) haciendo $n$ ! 1 se tiene lo siguiente

$$
\begin{aligned}
\prod_{k=0}^{X} Q_{\Pi}^{k}\left(F_{\Pi}(1)\right)+\lim _{n !} Q_{\Pi}^{n+1}(1) & =1 \\
1+\lim _{n !} m_{1} Q_{\Pi}^{n+1}(1) & =1
\end{aligned}
$$

de donde concluimos que $\lim _{n !} Q_{\Pi}^{n+1}(1)=0$.

2) implica 1)

Como

$$
\lim _{n !} Q_{\Pi}^{n+1}(1)=0
$$

entonces de la ecuación (3;13) se deduce también que

$$
\lim _{n !} \prod_{1} \prod_{k=0}^{x} Q_{\Pi}^{k}\left(F_{\Pi}(1)\right)=1
$$

pero ésto último unicamente indica que

$$
\mathbb{R}_{\Pi}^{\min }(1)=1
$$

o sea que expresada en su forma cuadrática, tenemos que

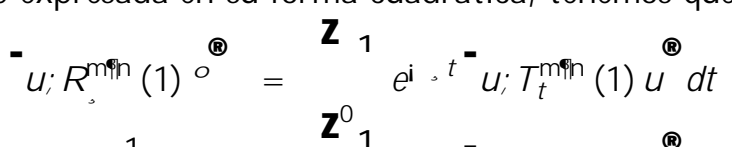

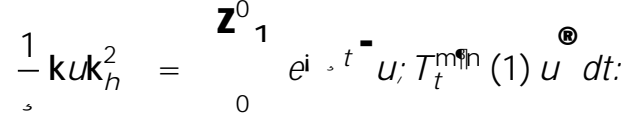

Pero por otra parte obsérvese lo siguiente

$$
\begin{aligned}
& \frac{1}{\Pi} \mathrm{kuk}_{\mathrm{h}}^{2}={ }_{0}^{\mathrm{Z}_{1}} \mathrm{e}^{\mathrm{i} \Pi \mathrm{t}} \mathrm{kuk_{h } ^ { 2 }} \mathrm{dt} \\
& \mathrm{Z}_{0} \mathrm{e}^{\mathrm{i} \Pi \mathrm{t}} \mathrm{kuk_{h } ^ { 2 } d t}={ }_{0}^{\mathrm{Z}_{1}} \mathrm{e}^{\mathrm{i} \Pi^{-}} \mathrm{u}_{;} T_{t}^{\operatorname{mm}}(1) \mathrm{u}^{\circledR} d t
\end{aligned}
$$

lo cual nos indica que

$$
\text { hu; ui }={ }^{-} u_{;} T_{t}^{\text {min }}(1) u^{\circledR} ;
$$


es decir $T_{t}^{m m n}(1)=1$ :

2) implica 3)

Supongamos que $\mathrm{Q}_{\Pi}(\mathrm{x})=\mathrm{x}$ :Vamos a demostrar que $\mathrm{x}=0$ :

Como $Q_{\Pi}$ es completamente positivo( Proposición;1;0;15), se tiene que

$$
\mathrm{Q}_{\Pi}\left(\mathrm{x}^{\mathfrak{x}}\right)=\left(\mathrm{Q}_{\Pi}(\mathrm{x})\right)^{\mathfrak{x}} \text { : }
$$

Gracias a esta igualdad, basta con suponer que $x$ es autoadjunto.

Puesto que

$$
\mathrm{i} \mathrm{kxk}_{1} 1 \cdot \mathrm{x} \cdot \mathrm{kxk}_{1} 1
$$

Empecemos por suponer que

$$
\mathrm{kxk}_{1} \cdot 1
$$

por lo tanto i $1 \cdot x \cdot 1 ; y$ al aplicar $Q_{\Pi}$ iteraramente

$$
\begin{gathered}
i Q_{\Pi}(1) \cdot x=Q_{\Pi}(x) \cdot Q_{\Pi}(1) \\
i Q_{\Pi}^{2}(1) \cdot x \cdot Q_{\Pi}^{2}(1) \\
\text { i } Q_{\Pi}^{n}(1) \cdot x \cdot Q_{\Pi}^{n}(1)
\end{gathered}
$$

si hacemos $\mathrm{n}$ ! 1 ; concluimos que $\mathrm{x}=0$ :

3) implica 2) Como

$$
\prod_{k=0}^{X n} Q_{\Pi}^{k}\left(F_{\Pi}(1)\right)+Q_{\Pi}^{n+1}(1)=1
$$

y $\Pi_{k=0}^{\mathrm{P}} \mathrm{Q}_{\Pi}^{\mathrm{k}}\left(\mathrm{F}_{\Pi}(1)\right)$ es una sucesión creciente y acotada de operadores acotados entonces converge ædébilmente. $E$ sto prueba que $\lim _{n !} Q_{\Pi}^{n+1}(1)$ existe, Ilamemos $x=\lim _{n !} Q_{\Pi}^{n+1}(1)$; entonces $Q_{\Pi}(x)=\lim _{n !} Q_{\Pi}^{n+1}(1)=x$; pero como por hipótesis tenemos que $x=0$; entonces $\lim _{n !} \prod_{1} Q_{\Pi}^{n+1}(1)=0$ :

Proposición 3.2.7 Supongamos la Hipótesis $A A$ y sea $\Pi>0$..jo. Entonces para todo $x 2 \mathrm{~B}(\mathrm{~h})$ se tiene que $\$(\mathrm{x})=\Pi \mathrm{x}$ si y sólo si $\mathrm{Q}_{\Pi}(\mathrm{x})=\mathrm{x}$ : Donde $\$(x)=\Pi x$; se entiende como igualdad entre formas sesquilineales, es decir para cada u; $i 2 \mathrm{D}(\mathrm{G}) ; \quad \$(\mathrm{x})[i ; \mathrm{u}]=\Pi h_{\mathrm{i}} ; \mathrm{xui}$ :

Demostración. Seguimos [8], pág.61.

Sea $\times 2 \mathrm{~B}(\mathrm{~h})$ positivo tal que $\$(\mathrm{x})=\Pi \mathrm{x}$ entonces para cada $¿ 2 \mathrm{D}(\mathrm{G})$ $y$ cada $t, 0$; tenemos lo siguiente:

$$
\$(x)\left[P_{t} i ; P_{t} i\right]=h P_{t} i ; x G P_{t} i i+h G P_{t} i ; x P_{t} i i+{ }_{=1}^{x} h L \cdot P_{t} i ; x L \cdot P_{t} i i:
$$


Pero, por otra parte:

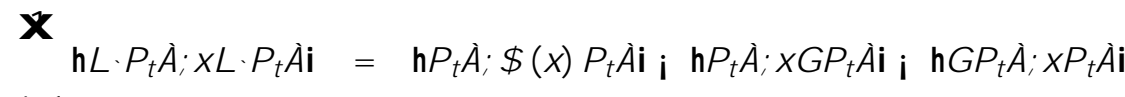

$$
\begin{aligned}
& =\prod h P_{t i} ; x P_{t i} i ; P_{t i} ; x G P_{t i} i ; h G P_{t} i ; x P_{t i} i:
\end{aligned}
$$

A hora bién, al tomar transformada de Laplace,

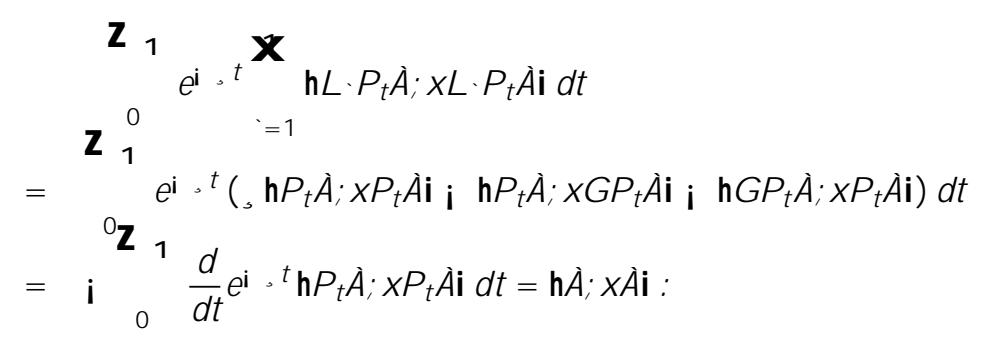

En otros términos tenemos que $h_{i} ; Q_{\Pi}(x) i i=h_{i} ; x_{i} i ;$ pero como $x, Q_{\Pi}(x)$ son operadores acotados y $D(G)$ es denso en $h$ entonces se tiene que $\mathrm{Q}_{\Pi}(\mathrm{x})=\mathrm{x}$. A hora veamos el recíproco: Sea $\mathrm{R}^{\mathrm{i}}{ }_{2} ; \mathrm{G}^{\phi}$ el operador resolvente ${ }^{i} \frac{{ }_{2}}{i} G^{\phi_{i}}{ }^{1}$. Nótese que para `, 1, el operador $L \cdot R^{i}{ }_{2} ; G^{\phi}$ tiene una extensión acotada y el operador $G R{ }^{i} \frac{\square}{2} ; G^{\dagger}$ puede ser extendido al operador acotado $\frac{\nabla_{2}}{2}{ }^{\mathrm{i}} \frac{\mathrm{D}}{2} ; \mathrm{G}^{\Phi}$ i 1 : Obsérvese que,

$\frac{d}{d t} L \cdot P_{t} R^{i} \frac{\square}{2} ; G^{\Phi} i=L \cdot R^{i} \frac{\square}{2} ; G^{\Phi} \frac{d}{d t} P_{t} i=i L \cdot P_{t} i+\frac{\square}{2} L \cdot P_{t} R^{i}{ }_{2} ; G^{\Phi} i \quad{ }^{i} 3 ; 13^{\phi}$ para cada ¿ $2 \mathrm{D}(\mathrm{G})$ : Por lo tanto, para cada i; $\int 2 \mathrm{D}(\mathrm{G})$. Sean ui =

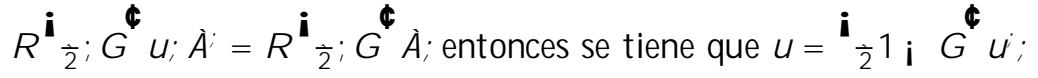
$i={ }^{i}{ }_{2} 1_{i} G^{\Phi}{ }^{i}$. Usando el Teorema Fundamental del Cálculo se obtiene 
lo siguiente

$$
\begin{aligned}
& x \\
& H \cdot u^{\prime} ; x L \cdot e^{\prime i} \\
& =i^{X^{X}} Z_{1} Z_{1} \frac{d}{d t} i^{i} e^{i t} h L \cdot P_{t} u^{i} ; x L \cdot P_{t} i^{i} i^{\Phi} d t \\
& =i^{X^{\pi} Z_{1}}{ }^{i} i \Pi^{i} \Pi^{\dagger}\left(L L \cdot P_{t} u^{i} ; x L \cdot P_{t} i j i\right) d t \\
& =1{ }^{0} \mathrm{Z}_{1} \\
& i^{x^{2}} e^{i} \Pi t \frac{d}{d t} h L \cdot P_{t} u^{i} ; x L \cdot P_{t} i ; i d t
\end{aligned}
$$

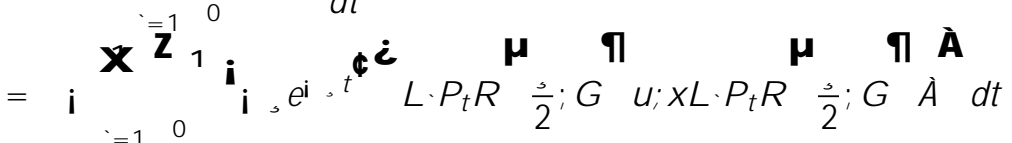

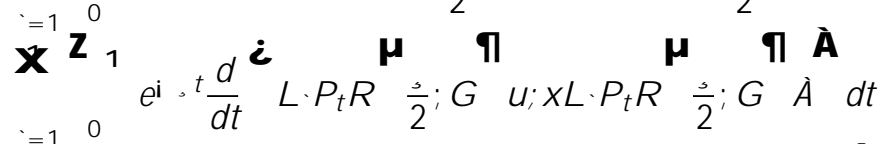

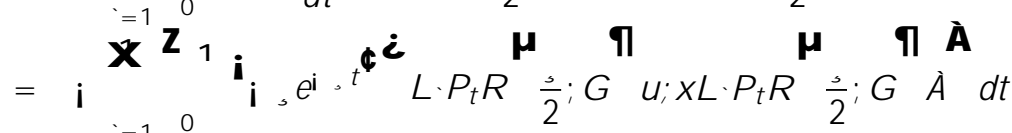

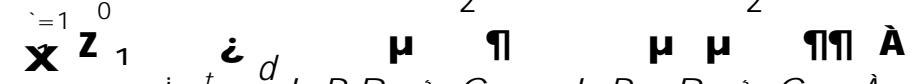

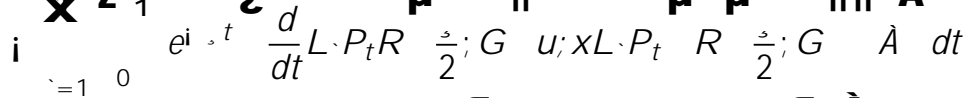

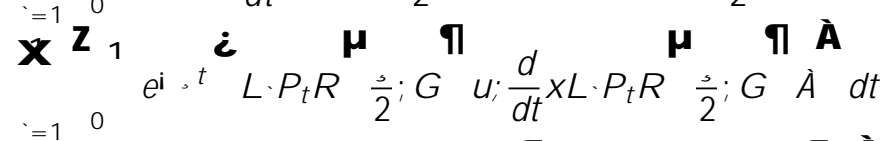

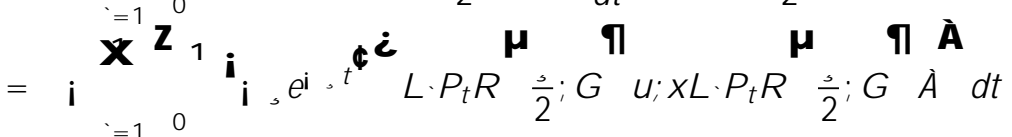

$$
\begin{aligned}
& x^{=1} Z_{1}^{0} e^{i \pi^{i}} i L \cdot P_{t} \int+\frac{\Pi}{2} L \cdot P_{t} R \stackrel{\mu}{2} ; G \quad u ; x L \cdot P_{t} R{ }_{\frac{\Pi}{2} ; G}^{\mu} i d t
\end{aligned}
$$

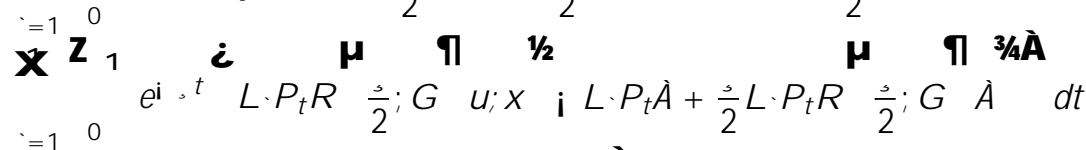

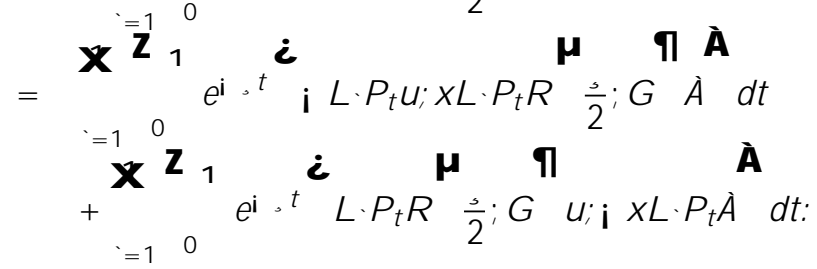


Por lo tanto,

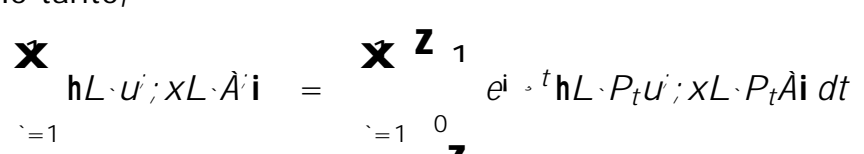

$$
\begin{aligned}
& +{ }_{=1}^{x} Z_{1}^{Z_{1}} e^{i t} h L \cdot P_{t} u ; x L \cdot P_{t} i j i d t \\
& =h u ; Q_{\Pi}(x) \dot{i}^{i} i+h u^{i} ; Q_{\Pi}(x) \dot{L}^{i}:
\end{aligned}
$$

Pero como $\mathrm{Q}_{\Pi}(\mathrm{x})=\mathrm{x}$; se tiene que esto último es igual a:

$$
\begin{aligned}
& \frac{\Pi_{2}}{2} \text { u }^{\prime} ; x_{i} ; i \text { i hGu'; } x_{i} ; i+\frac{\Pi_{2}}{2} h^{\prime} ; x_{i} ; i \text { i hu; } x_{i} ; i
\end{aligned}
$$

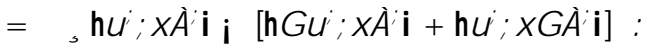

Deduciéndose así que,

$$
\begin{aligned}
& x
\end{aligned}
$$

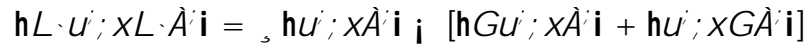

$$
\begin{aligned}
& =1
\end{aligned}
$$

y usando ahora la de.nición de $\$(x)$; se tiene lo siguiente:

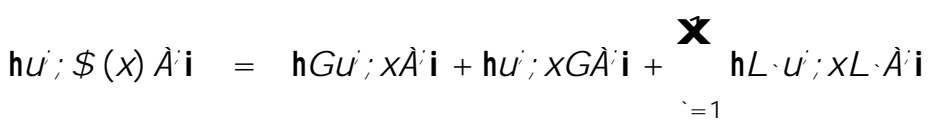

$$
\begin{aligned}
& =h u^{i} ; x_{i} ; i+h^{i} ; x_{i} ; i+\Pi_{h i} ; x_{i} ; i \\
& \text { i [hGu'; } \left.x_{i} ; i+h u^{\prime} ; x_{i} ; i\right] \\
& =\Pi u^{i} ; x_{i} i i ;
\end{aligned}
$$

es decir, hemos probado que hi $; \$(x) i^{i} i=h^{i} ; \prod_{\chi^{\prime}} ; i$ :

Como $\int_{i} ; i$ son arbitrarios en $D(G)$ entonces $i^{;} ; u^{i}$ son arbitrarios en $D^{i} G^{2}{ }^{\phi}$ y puesto que $D^{1} G^{2}$ es esencia de $G$ concluimos que hu; $\$(x) i i=h u ; \Pi x i i$; es decir $\$(x)=\Pi x$ :

Proposición 3.2.8 Supongamos que se tiene la Hipótesis $A A$, para $\Pi>0$ ..jo. Las siguientes condiciones son equivalentes:

1) El Semigrupo Dinámico Cuántico $T^{m \text { min }}$ es conservativo;

2) No existe $x 2 \mathrm{~B}(\mathrm{~h})$ distinto de 0 tal que $\$(\mathrm{x})=\Pi \mathrm{x}$ :

Demostración. Seguimos [8], pág.62.

1) implica 2)

Supongamos que $T^{\text {min }}$ es conservativo entonces por el Teorema 3;2;7,

$$
\mathrm{Q}_{\Pi}(\mathrm{x})=\mathrm{x} \text { si } \mathrm{y} \text { sólo si } \mathrm{x}=0 \text { : }
$$

y por la Proposición anterior no existe $\times 2$ B (h) distinto de 0 tal que 
$\$(\mathrm{x})=\Pi \mathrm{x}:$

2) implica 1)

Supongamos que la única solución x 2 B (h) a la ecuación $\$(x)=\Pi x$ es $x=0$; entonces por 2) del Teorema anterior la a..rmación 1) queda demostrada.

Finalizamos esta sección y por consiguiente el capítulo, con dos importantes proposiciones sobre Semigrupos Dinámicos Cuánticos Conservativos.

Proposición 3.2.9 Supongamos la Hipótesis A A. El subespacio lineal $V$ generado por jiihuj; $u_{i} i 2 \mathrm{D}(\mathrm{G})$ está contenido en el dominio del generador in...nitesimal $\$$ del semigrupo predual $S^{m * n}$ de $T^{m \text { m }}$ y

$$
\$_{x}(j i \text { ihuj })=j G_{i} i h u j+{ }_{`=1}^{x} j L \cdot i i h L \cdot u j+j i i h G u j
$$

donde la serie converge en la norma de la traza. Las siguientes condiciones son equivalentes:

1) EI subespacio lineal generado $V$ es esencia de $\$$;

2) El Semigrupo Dinámico Cuántico Minimal $T^{\text {min }}$ es conservativo.

Demostración.

Seguimos [8], págs.62-64.

Para cada i; u 2 D (G) y cada x 2 B (h), la ecuación:

$$
h_{i} ; T_{t}(x) \text { ui }=h_{i} ; x u i+{ }_{0}^{Z_{t}} \$\left(T_{s}(x)\right)[i ; u] d s
$$

puede ser escrita como:

$$
\begin{aligned}
& \operatorname{tr}^{\mathrm{i}} \mathrm{S}_{\mathrm{t}}^{\mathrm{mm}}\left(\mathrm{j} \dot{\mathrm{i} i h u j} \mathrm{z}_{\mathrm{t}}{ }^{\phi}\right.
\end{aligned}
$$

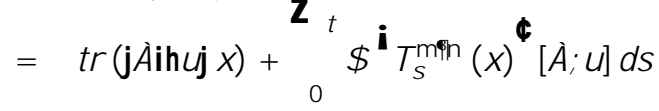

de donde

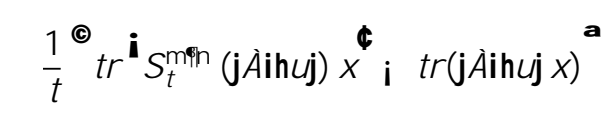

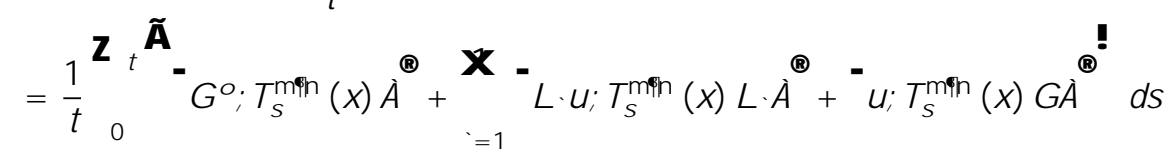

ahora haciendo t! Q Q $Q^{+}, y$ por la continuidad de las funciones,

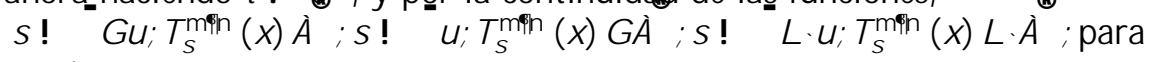
, 1 tenemos que:

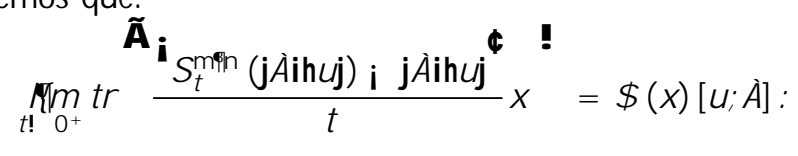


Esto muestra que j¿ihuj está en el dominio del generador débil de ${\stackrel{C}{S_{t}^{m}}{ }^{m} \underline{a}}_{t, 0}$ que actua sobre $\varnothing(\mathrm{h})$; y como el generador fuerte y el generador débil coin-

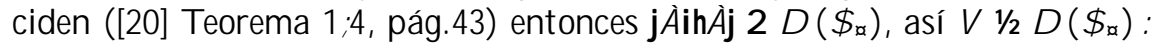

A hora demostramos que:

1) implica 2)

Nótese que para cada $\Omega 2 \mathrm{~V}$; se tiene que $\operatorname{tr}\left(\$_{\alpha}(\Omega)=0\right.$; pues usando la

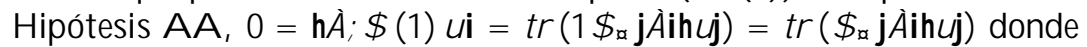
¿; u $2 \mathrm{D}(\mathrm{G})$ : Pero siendo $\mathrm{V}$ una esencia de $\$$, es claro que también se tiene para todo $\Omega 2 \mathrm{D}(\$ x)$ : Pues estos $\Omega$ pueden ser aproximados por elementos de $\mathrm{V}$ :

Con lo cual se tiene que:

$$
\frac{\mathrm{d}}{\mathrm{dt}} \operatorname{tr}^{\mathrm{i}} \mathrm{S}_{\mathrm{t}}^{\operatorname{mm}}\left(\Omega \Omega^{\Phi}=\operatorname{tr}^{\mathrm{i}} \$_{\alpha}{ }^{\mathrm{i}} \mathrm{S}_{\mathrm{t}}^{\operatorname{mmn}}(\Omega)^{\phi థ}=0\right.
$$

lo cual demuestra que

$$
\operatorname{tr}^{\mathrm{i}} \mathrm{S}_{\mathrm{t}}^{\mathrm{min}}(\Omega)^{\Phi}=\text { Cte }
$$

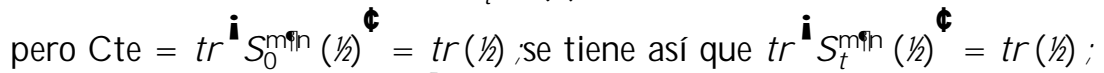
por lo tanto resulta que $T^{\mathrm{m} m}$ es conservativo.

2) implica 1)

A hora supongamos que $T^{m \text { m }}$ es conservativo. Vamos a demostrar que $V$ es esencia de $f_{x}$ : Pero esto es cierto si y sólo si $(\Pi ; \$$ Pues supongamos que $\left(\Pi ; \$_{\alpha}\right)(V)$ es denso en $\varnothing(h)$ entonces dado $\Omega 2$

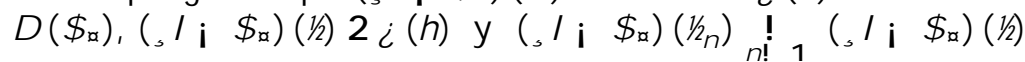
con $f \Omega_{n} g_{n} 1 / 2 \mathrm{~V}$, por lo tanto:

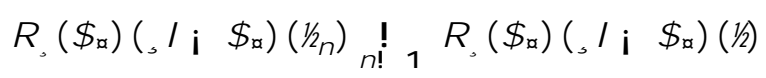

lo cual implica que

$$
\Omega_{n} ! 1 \Omega \text { y }\left(\Pi \text { i } \$_{x}\right)\left(\Omega_{n}\right)=\Pi\left(\Omega_{n}\right) \text { i } \$_{x}\left(\Omega_{1}\right) ;
$$

pero de esto último se tiene que:

$$
\${ }_{x}\left(\Omega_{1}\right)=\Pi\left(\Omega_{1}\right) \text { i }\left(\Pi ; \quad \$_{x}\right)\left(\Omega_{1}\right)
$$

así $\$_{x}\left(\Omega_{1}\right)_{n !}{ }_{n} \$_{x}(\Omega)$; por lo tanto $V$ es esencia de $\$_{x}$ :

A hora sea $\times 2 B(h)$ entonces tomemos $i_{x}$ el funcional en $\varnothing(h)$ de..nido por $i_{x}\left(\left(\Pi{ }_{i} \$_{x}\right) j u i h_{i} j\right)=\operatorname{tr}\left(\left(\Pi i \$_{x}\right)(x) j u i h_{i} j\right)$; veremos que $i_{x}$ es el fun- 
76CAPÍTULO 3. EL SEMIGRUPO DINÁMICO CUÁNTICO MINIMAL (S.D.C.M.)

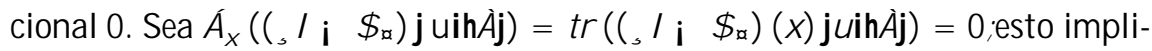
ca que

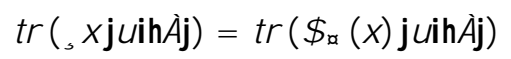

es decir

$$
\text { hu; } \Pi x_{i} i=h u ; \$ x(x) i i:
$$

Lo cual dice que $\$_{x}(x)=\Pi x$, pero siendo $T^{m \text { min }}$ conservativo esto es cierto si $\mathrm{x}=0$; así el anulador de $\left(\Pi_{\mathrm{i}} \$_{x}\right)(\mathrm{V})$ es igual a $\mathrm{f} 0 \mathrm{~g}$ :

Lema 3.2.10 Considerando $\$^{\min }$ y su predual $\$_{x^{m}}^{\min }$. Se tiene que $D^{i}\left(\$_{x}^{\operatorname{mm}}\right)^{x^{\infty}}=$ $\mathrm{D}\left(\$^{\mathrm{m} m}\right)$ :

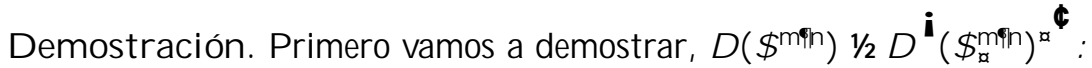

Sea $\times 2 D\left(\$^{m i n}\right)$ y sea $\Omega 2 D\left(\$ m_{x}^{m}\right)$; tenemos que demostrar que

$$
\Omega ! \operatorname{tr}^{i} \$ m_{x}^{m+n}(\Omega) x^{\phi}
$$

es una funcional lineal acotada.

Por otra parte tenemos que

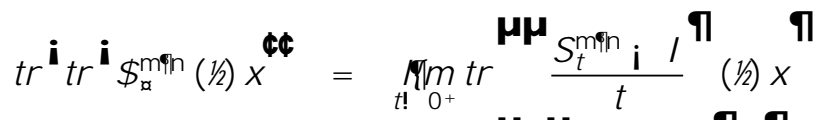

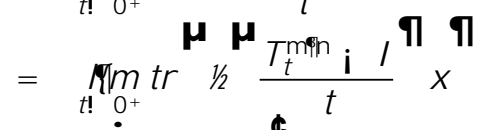

$$
\begin{aligned}
& =\operatorname{tr}^{i} \Omega^{\min }(\mathrm{x})^{\phi}
\end{aligned}
$$

por lo tanto

$$
j \operatorname{tr}^{i} \Omega{ }^{m+n}(x)^{\Phi} j \cdot j j \Omega j j_{t r} j j \$^{m i n}(x) j j
$$

resultando así que la funcional

$$
\Omega ! \operatorname{tr}^{i} \$ m_{x}^{m+n}(\Omega) x^{\phi}
$$

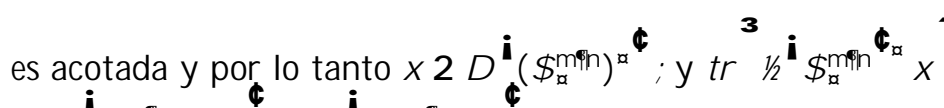

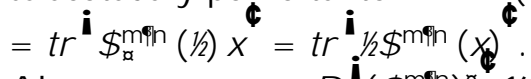

A hora veamos que $\mathrm{D}^{\mathrm{i}}\left(\$_{\alpha}^{\min }\right)^{\infty}{ }_{1 / 2} \mathrm{D}^{\mathrm{i}} \$_{\mathrm{mm}}{ }^{\phi}$ :

En este caso haremos iuso del Teorema de Hille Yosida ([3];Pág. 170).

Como $D_{\phi}^{1} \$^{m+n}{ }_{1 / 2} D^{i}\left(\$_{p}^{m m n}\right)^{a} ; y_{\alpha}$ puesto que $\$ m^{m i n}$ genera un semigrupo, $D^{1} \$^{m i n}$ es denso así $D\left(\$ m_{\alpha}^{m}\right)^{\infty}$ es denso en $\varnothing(h)$ : Demostramos que

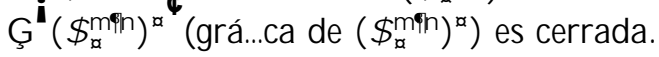

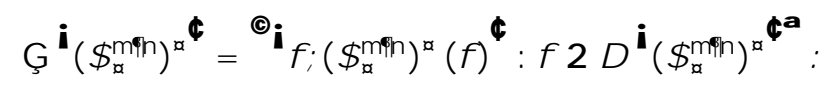

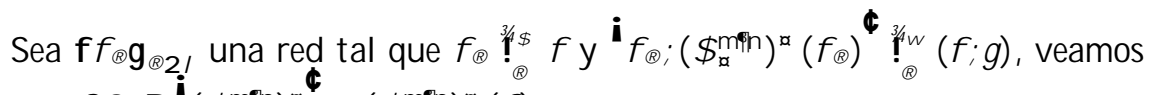

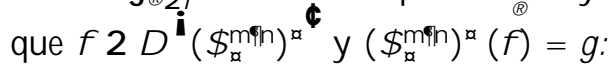


Tomemos $\Omega 2 D^{i}\left(\$_{\alpha}^{m}\right){ }^{\phi}$ y sea la funcional $\Omega ! \operatorname{tr}^{i}\left(\$_{x}^{\min }\left(\Omega f^{\phi}\right.\right.$;

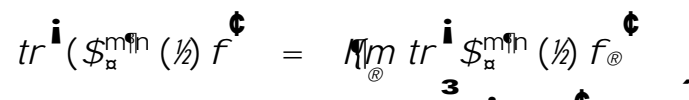

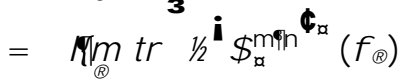

$$
\begin{aligned}
& =\operatorname{tr}(\Omega \mathrm{g}) \text {; }
\end{aligned}
$$

así. la funcignal $\Omega$ ! $\operatorname{tr}^{\mathrm{i}}\left(\$_{\alpha}^{\min }\left(\Omega \mathrm{f}^{\phi}\right.\right.$ resulta acotada, con lo gual f 2

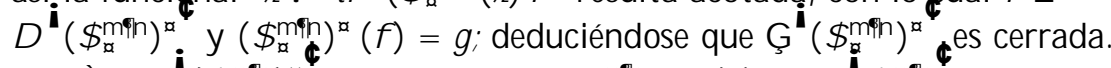
Sea $i 2 D^{i}\left(\$ m_{x}^{m n}\right)^{a}$, recuérdese que $R_{k}^{m m}: B(h) ! D^{1} \$^{m m n}$ : Vamos a demostrar que

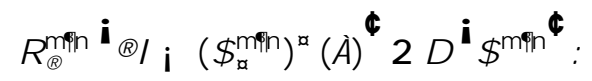

Tomemos $\Omega 2 \varnothing(\mathrm{h})$ arbitrario.

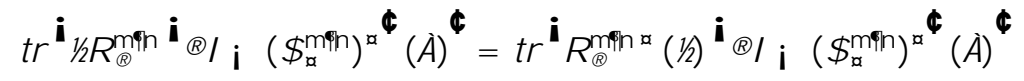

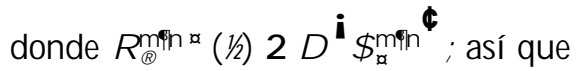

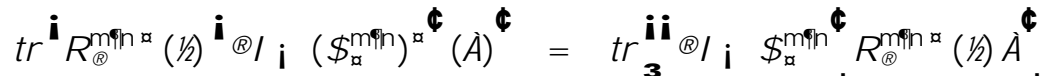

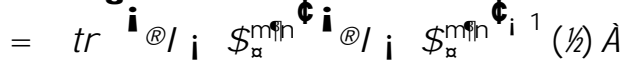

$$
\begin{aligned}
& =\operatorname{tr}\left(\Omega_{i}\right)
\end{aligned}
$$

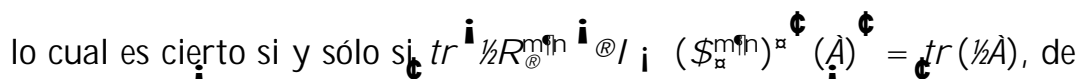

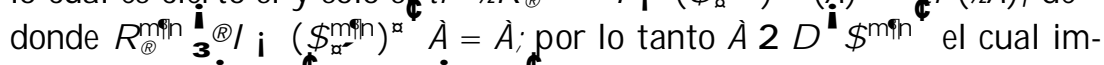

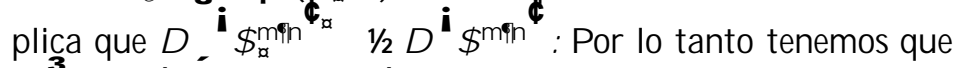
$D^{i \operatorname{man}_{x} \phi_{\alpha x}}=D^{i} \$^{m i n}{ }^{\phi}$ :

Proposición 3.2.11 Supóngase que se tiene la Hipótesis AA. Entonces las siguientes condiciones son equivalentes.

1) $T^{\text {min }}$ es conservativo;

2) $D\left(\$ m^{m i n}\right)=f \times 2 B(h): \$(x)$ es una forma sesquilineal acotadag.

Demostración. 1) implica 2)

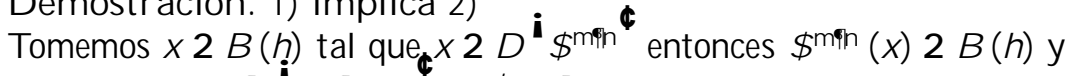
se tiene que $\$^{m^{m}}{ }^{i} T_{t}^{m i n}(x)^{4}=\frac{d}{d t} T_{t}^{m m}(x)$, donde la derivada se toma en la topología ædébil. Esto implica que para todo ¿; u 2 D (G)

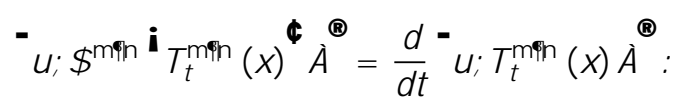




\section{CAPÍTULO 3. EL SEMIGRUPO DINÁMICO CUÁNTICO MINIMAL (S.D.C.M.)}

Por otra parte también se tiene que:

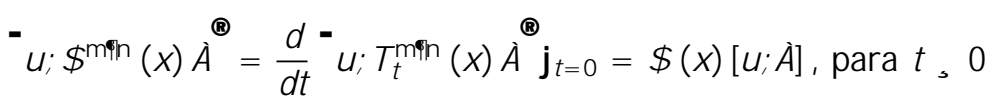

de donde se deduce fácilmente que

$$
\mathrm{u} ; \$^{\min }(\mathrm{x}) i^{\circledR}=\$(\mathrm{x})[\mathrm{u} ; i] \text { : }
$$

Por lo tanto la forma sesquilineal de $\$(x)$ coincide sobre $D(G) £ D(G)$ con la forma sesquilineal del operador $\${ }^{m i n}(x)$, por lo tanto $\$(x)$ es acotada.

Esto demuestra

$D\left(\$ m^{m n}\right) 1 / 2 f x 2 B(h): \$(x)$ es una forma sesquilineal acotadag.

Recíprocamente, si \$ $(x)$ es acotada entonces existe $Y 2$ B (h) tal que:

$$
\$(x)[u ; i]=h u ; Y_{i} i
$$

para todo u; ¿ $2 \mathrm{D}(\mathrm{G}):$
Para probar que $\times 2 \mathrm{D}\left(\$^{\mathrm{mm}}\right)$ : Considérese la funcional $\Omega$ ! $\operatorname{tr}^{\mathrm{i}} \$_{\mathrm{x}}^{\operatorname{mm}}(\Omega) \times{ }^{\phi}$

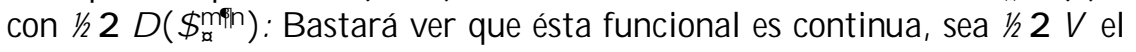
siguiente operador de traza ...nita

$$
\Omega=X_{i=1}^{d} j u_{i} i h_{i j} j
$$

con $u_{i} ;$ i 2 D (G) :

Entonces

$$
\begin{aligned}
& \operatorname{tr}^{i} \$_{x}^{\min }\left(\Omega x^{\phi}={ }_{i=1}^{x^{d}} \operatorname{tr}^{i} \$_{x}^{m i n}\left(j u_{i} i h_{i j} j\right) x^{\phi}={ }_{i=1}^{x^{d}} \$(x)\left[u_{i} ; i_{i}\right]\right. \\
& =X_{i=1}^{d} h u_{i} ; Y_{i i} i=X_{i=1}^{d} \operatorname{tr}\left(Y j u_{i} i h_{i}\right)=\operatorname{tr}(Y \Omega
\end{aligned}
$$

por lo tanto

$$
j \operatorname{tr}{ }^{i} \$_{\alpha}^{m m}(\Omega) x_{j}^{\phi} \cdot j j Y j_{j}(B(h)) j j \Omega j j_{t r}
$$

lo cual indica que $\times 2^{i} D\left(\$_{x}^{m}\right)^{x}$, usando el lema anterior se tiene que $\times 2 \mathrm{D}\left(\$ \mathrm{~m}^{\mathrm{min}}\right)$ :

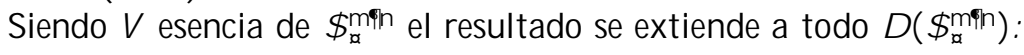

2) implica 1)

Como $\$(I)[\mathrm{l} ; i]=0$; por la Hipótesis $A$ A, u; 2 $2 \mathrm{D}(\mathrm{G})$ se deduce que I $2 \mathrm{D}^{\mathrm{i}} \$ \mathrm{~min}$ :

A hora para todo $\Pi>0$;

$$
\begin{aligned}
& \mathrm{R}_{\Pi}^{\min }{ }^{\mathrm{i}} \Pi+\$^{\min ^{\phi}}(\mathrm{I})=\mathrm{I} \\
& \mathrm{R}_{\Pi}^{\min } \mathrm{i} \Pi 1+\$^{\min }(\mathrm{I})^{\Phi}=\mathrm{I}
\end{aligned}
$$


lo que implica que

$$
\mathbb{R}_{\Pi}^{\min }(\mathrm{I})=\mathrm{I}:
$$

Para todo u $2 \mathrm{~h}$

$$
\begin{aligned}
& j_{j u j j} j_{h}^{2}=\text { hij }_{1} 1 u i=\Pi^{-} u ; R_{\Pi}^{m i n}(I) u^{\circledR} \\
& =\prod_{0} e^{i \Pi^{-}} u ; T_{s}^{m m}(I) u^{\circledR} d s \\
& \text { y } \quad \frac{j j u j j_{h}^{2}}{\Pi}={ }_{0}^{Z_{1}} e^{i \Pi^{-}} u ; T_{s}^{m m n}(I) u^{\circledR} d s
\end{aligned}
$$

lo cual quiere decir que

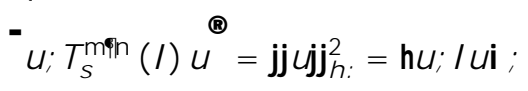

por lo tanto se tiene

$$
\mathrm{T}_{\mathrm{s}}^{\mathrm{mmin}}(1)=1 \text {; para todo } \mathrm{s}, 0 \text { : }
$$


80CAPÍTULO 3. EL SEMIGRUPO DINÁMICO CUÁNTICO MINIMAL (S.D.C.M.) 


\section{Capítulo 4}

\section{Criterios de Conservatividad}

En este capítulo damos condiciones su..cientes para la conservatividad de un S.D.C.M. A demás de la hipótesis A A usada en el capítulo anterior supondremos válida la hipótesis C (pág.82). A mbas hipótesis permiten demostrar la conservatividad de un S.D.C.M. 


\subsection{Hipótesis C}

Sean G; L. (’ , 1) operadores que satisfacen la H ipótesis A A. Estos operadores satisfacen la Hipótesis $C$ si existe un operador, densamente de..nido, positivo, autoadjunto, $C$ y un subespacio lineal $D$ con las siguientes propiedades:

1) D está contenido en el dominio de G:

2) $D$ es esencia de $C^{\frac{1}{2}}$ :

3) $D$ es invariante bajo el semigrupo $f P_{t} g_{t}$, o y el subespacio $R(\Pi G)$ (D) está contenido en el dominio de $C^{\frac{1}{2}}$ para cada $\Pi>0$ :

4) Para cada ` $2 N, \Pi>0$, el subespacio $L \cdot R(\Pi G)(D) 1 / 2 D\left(C^{\frac{1}{2}}\right)$ :

5) Existe una constante positiva b tal que:

$$
2 \operatorname{Re}^{\mathrm{D}} \mathrm{C}^{\frac{1}{2}} i ; C^{\frac{1}{2}} \mathrm{G}_{i}{ }^{\mathrm{E}}{ }^{{ }^{*}=1} \mathrm{C}^{\frac{1}{2}} \mathrm{~L} \cdot i ; C^{\frac{1}{2}} \mathrm{~L} \cdot i^{\mathrm{E}} \cdot \mathrm{bjj} \mathrm{C}^{\frac{1}{2}} i j \mathrm{j}_{\mathrm{h}}^{2}
$$

para cada ¿ $2 R(\Pi G)(D)$ y para algún $\Pi>0$ :

N ota:

Obsérvese que de la identidad $G R(\Pi G)=\Pi R(\Pi G)$; 1 ([18]; págs;36 ; 37), se tiene que el subespacio lineal $G R(\Pi G)(D)$ está contenido en el dominio de $C^{\frac{1}{2}}$. Por lo tanto si u $2 \mathrm{R}(\Pi \mathrm{G})(\mathrm{D})$; existe ¿ $2 \mathrm{D}$ tal que

$$
\mathrm{u}=\mathrm{R}(\Pi \mathrm{G}) \text { i y } \mathrm{Gu}=\mathrm{GR}(\Pi \mathrm{G}) \dot{2} 2 \mathrm{D}\left(\mathrm{C}^{\frac{1}{2}}\right):
$$

Como consecuencia $C^{\frac{1}{2}} \mathrm{G} \int$ tiene sentido en $(4 ; 1)$ : También denotamos por $\mathrm{k}: \mathrm{k}_{\mathrm{C}} \frac{1}{2}$ la norma en el dominio de $\mathrm{C}^{\frac{1}{2}}$ dada $_{\mathrm{o}}$ por

$$
k_{i} k_{C}^{2} \frac{1}{2}=k_{i} k_{h}^{2}+{ }^{\circ} \mathrm{C} \frac{1}{2} i^{\circ 2}{ }^{2}
$$

Esta norma proviene del producto interno:

$$
h_{i} ; \pi i{ }_{C} \frac{1}{2}=h_{i} ; \pi i+{ }^{D} C^{\frac{1}{2}}{ } ; C^{\frac{1}{2}} \pi
$$

$\sum ; \pi 2 \mathrm{D}\left(\mathrm{C}^{\frac{1}{2}}\right)$; que hace de $\mathrm{D}\left(\mathrm{C}^{\frac{1}{2}}\right)$ un espacio de Hilbert. Esta nueva norma induce una topología estrictamente más fuerte que la que induce la norma original. Denotemos por $\mathrm{k}: \mathrm{k}_{\mathrm{C} ; 1}$; la norma de operadores en este nuevo espacio de Hilbert. 
El siguiente resultado es una estimación sobre $R(n ; G)$, el operador resolvente $(\mathrm{nl} ; \mathrm{G})^{\mathrm{i}}{ }^{1}$ en $\mathrm{h}$ :

Proposición 4.1.1 El operador ${ }^{P}{ }_{i=1}^{1}(n L \cdot R(n ; G))^{\infty}(n L \cdot R(n ; G))$; de..nido en $\mathrm{h}$; satisface:

$$
j^{X} m L \cdot R(n ; G) i ; n L \cdot R(n ; G) i i j \cdot 4 n j j i j j{ }_{h}^{2}:
$$

Demostración.

Seguimos [8], pág.66.

Sea ¿ $2 \mathrm{D}(\mathrm{G})$; entonces se tiene debido a la Hipótesis $A$,

${ }^{X}{ }^{X} m L \cdot R(n ; G) i ; n L \cdot R(n ; G) i i j \quad . \quad 2 j m R(n ; G) i ; n G R(n ; G) i j j$

- $\quad 2 j j n R\left(n_{f} G\right) i j j h$ jjnGR (n;G) $\dot{c} j j h$

- $\quad 2 j j i j j j_{h}^{2} n^{2} j j R(n ; G) i j j_{h}+n$

- $\quad 2 \mathrm{jj} i \mathrm{jj} \mathrm{h}_{\mathrm{h}}^{2} ; \mathrm{n}$

$=4 n j j i j j_{\mathrm{h}}^{2}$ :

A hora denotemos por $\mathrm{J}$ n el operador positivo que satisface

X $\mathrm{mL} \cdot \mathrm{R}(\mathrm{n} ; \mathrm{G}) \dot{i} ; \mathrm{nL} \cdot \mathrm{R}(\mathrm{n} ; \mathrm{G}) \dot{i}=h_{i} ; J_{n} \dot{i}^{\mathrm{i}}$ :

Proposición 4.1.2 Supongamos la Hipótesis A A. Para cada ¿ 2 h; se tiene lo siguiente:

$$
{ }_{k=1}^{X}-i ; Q_{\Pi}^{k}(1) i^{\circledR} \cdot \lim _{n ! 1} \inf ^{-} i ; R_{\Pi}^{\min }\left(J_{n}\right) i^{\circledR}:(4 ; 2)
$$

Demostración. Seguimos [8], pág.66.

$$
\begin{aligned}
& \text { Para ¿ } 2 \text { D y n, } 1 \text { se satisface: } \\
& h_{i} ; F_{\Pi}\left(J_{n}\right) \dot{i}=e^{i{ }^{i}} h P_{s} i ; J_{n} P_{s} i i d s \\
& =Z_{1}^{0} e^{i{ }^{x}}{ }^{X} m L \cdot P_{s} R(n ; G) i ; n L \cdot P_{s} R(n ; G) i i d s \\
& \mathrm{Z}^{0} \quad{ }^{0}=1 \\
& =Z_{0}^{Z_{1}} e^{e^{i}{ }^{X}} j j n L \cdot R(n ; G) P_{s} i j j_{h}^{2} d s \\
& =x_{=1}^{Z_{1}} e^{\mathrm{e}^{i s_{j j}} \mathrm{jL} \cdot R(n ; G) P_{s} i j j j_{h}^{2} d s} \\
& =\quad m R(n ; G) i ; n Q_{\Pi}(1) R(n ; G) \dot{i}{ }_{\circledast} \\
& =\quad i ; n^{2} R\left(n ; G^{\ltimes}\right) Q_{\Pi}(1) R(n ; G) i
\end{aligned}
$$


resultando así que $F_{\Pi}\left(J_{n}\right)=n^{2} R\left(n ; G^{\circledR}\right) Q_{\Pi}(1) R(n ; G)$ :

También tenemos acotación es uniforme para $F_{\Pi}\left(J_{n}\right)$; pues

$$
\begin{aligned}
j j F_{\Pi}\left(J_{n}\right) j j_{B(h)}= & j j n^{2} R\left(n ; G^{\bowtie}\right) Q_{\Pi}(1) R(n ; G) j j \\
\cdot & n^{2} j j R\left(n ; G^{\ltimes}\right) j j: j j Q_{\Pi}(1) j j_{B(h)}: j j R(n ; G) j j \\
\cdot & n^{2} \frac{1}{n^{2}} j j Q_{\Pi}(1) j j_{B(h)} \\
\cdot & j j Q_{\Pi} j_{B}(B(h)) \\
\cdot & 1:
\end{aligned}
$$

Por lo tanto para todo $\mathrm{n} 2 \mathrm{~N}$ se tiene $\mathrm{jjF}_{\Pi}\left(\mathrm{J}_{\mathrm{n}}\right) \mathrm{jj}_{\mathrm{B}(\mathrm{h})} \cdot \mathrm{l}$ :

Como $n^{2} R\left(n ; G^{\infty}\right) Q_{\Pi}(1) R(n ; G)=n R\left(n ; G^{\infty}\right) Q_{\Pi}(1) n R(n ; G)$ y $n R(n ; G), n R\left(n ; G^{\infty}\right)$ ambos convergen fuertemente a I;cuando $n$ ! 1 ([20]; Lema 3;2; pág;9). Entonces $\mathrm{Q}_{\Pi}(1) \mathrm{nR}(\mathrm{n} ; \mathrm{G}) ! \mathrm{Q}_{\Pi}(1)$ fuertemente, así resulta que $\mathrm{F}_{\Pi}\left(\mathrm{J}_{\mathrm{n}}\right)_{n !}{ }_{1} \mathrm{Q}_{\Pi}(1)$ fuertemente.

A hora sea $\int 2 \mathrm{D}$ y usando que $\mathrm{Q}_{\Pi}^{\mathrm{k}}$ es normal se tiene que:

$$
\lim _{n !}{ }^{-} \int ; Q_{\Pi}^{k} F_{\Pi}\left(J_{n}\right) \int^{\circledR}=-\int ; Q_{\Pi}^{k+1}(1) \int^{\circledR}:
$$

Pero por el Lema de Fatou, se deduce también que:

$$
x_{k=0}^{X}-\int ; Q_{\Pi}^{k+1}(1) \int^{\circledR} \cdot \lim _{n !} \inf _{k=0}{ }^{X}-\int_{n} ; Q_{\Pi}^{k} F_{\Pi}\left(J_{n}\right) \int^{\circledR}
$$

obteniéndose así,

$$
X_{k=1}^{X}-\int_{\Pi} ; Q^{k}(1) \int^{\circledR} \cdot i_{n !} \operatorname{minf}_{1}^{-} \int ; R_{\Pi}^{\min }\left(J_{n}\right) \int^{\circledR}
$$

y como $D$ es denso en $h$, el resultado se extiende a todo $h$.

Con esta proposición demostrada podemos preguntarnos ¿Cuándo la serie en (a) converge?. Para ello sólo será necesario estimar a $R_{\Pi}^{m \text { mn }}\left(J_{n}\right)$ : Pero esto es posible si para " $>0$ se tiene que $\mathrm{J}_{\mathrm{n}} \cdot \mathrm{C}_{1} \mathrm{y}$

$$
\operatorname{Sup}_{n, 1} i ; R_{\Pi}^{\min }\left(\mathrm{J}_{\mathrm{n}}\right) i^{\circledR} \text {. } \operatorname{Sup}_{\text {" }>0}{ }^{-} i ; R_{\Pi}^{\min }\left(\mathrm{C}_{n}\right) i^{\circledR}<1
$$

para $i$ en un subconjunto denso de $\mathrm{h}$; siendo $\mathrm{C}$ un operador positivo autoadjunto y los $C_{\text {" }}$ operadores acotados,

$$
\mathrm{C}_{1 "}=\mathrm{C}(1+\mathrm{C})^{\mathrm{i}}{ }^{1} \text { : }
$$

Los operadores C" son las aproximaciones de Yosida para C: 
Lema 4.1.3 Supongamos la Hipótesis A A y la Hipótesis C . Entonces para cada $\Pi>$ b; $D\left(C^{\frac{1}{2}}\right)$ es invariante bajo $R(\Pi G)$ y además se tiene que:

$$
\mathrm{kR}(\Pi \mathrm{G}) \mathrm{k}_{\mathrm{c} ; 1} \cdot\left(\Pi_{i} b^{b}\right)^{{ }^{1}}{ }^{1}
$$

Demostración. Seguimos [8], pág.68.

A hora pasamos a demostrar primero la desigualdad. Para ello tomemos ¿ 2 D;y sea $u=\Pi R(\Pi G) i=\Pi(\Pi \text { i G })^{i}{ }^{1} i$ entonces $i=\frac{1}{\Pi}(\Pi$ i G $) u$ $={ }^{1} 1 \mathrm{i} \Pi^{1}{ }^{1} \mathrm{G}$ u: Por lo tanto,

$$
\begin{aligned}
& j j C^{\frac{1}{2}} i j j_{h}^{2}=j i C^{\frac{1}{2}} i_{1} i \Pi^{1} G^{\phi} u j j_{h}^{2} \\
& =C^{\frac{1}{2}} u_{i} \Pi^{1} C^{\frac{1}{2}} G u ; C^{\frac{1}{2}} \int i \Pi^{1} C^{\frac{1}{2}} G u^{E} \\
& =j j C^{\frac{1}{2}} u j j \mathrm{~h}_{\mathrm{h}}^{2} \mathrm{i} 2 \Pi^{1}{ }^{1} \operatorname{Re} C^{\frac{1}{2}} \mathrm{u} ; \mathrm{C}^{\frac{1}{2}} \mathrm{Gu}+\Pi^{2}{ }^{2 \mathrm{jj} C^{\frac{1}{2}} G u j j_{\mathrm{h}}^{2}}
\end{aligned}
$$

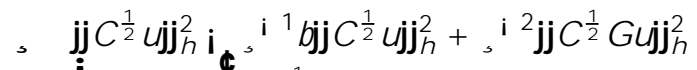

$$
\begin{aligned}
& \text { - }{ }^{i} 1_{i} \Pi^{1} b^{\Phi} j j C^{\frac{1}{2}} u j j_{h}^{2} \\
& ={ }^{i}{ }_{1} \Pi^{1}{ }^{1} b^{\phi} j j C^{\frac{1}{2}} \Pi R(\Pi G) i j j h_{h}^{2} \\
& =\Pi^{2}{ }^{i} i_{i} \Pi^{1} b^{4} j j C^{\frac{1}{2}} R(\Pi G) i j j_{h}^{2} \\
& =\Pi(\Pi i b) j j C^{\frac{1}{2}} R(\Pi G) i j j_{h}^{2} \text { : }
\end{aligned}
$$

Así

$$
\Pi(\Pi i \quad b) j j C^{\frac{1}{2}} R(\Pi G) i j j h_{h}^{2} \cdot j j C^{\frac{1}{2}} i j j h
$$

con lo cual,

$$
j j C^{\frac{1}{2}} R(\Pi G) i j j_{h}^{2} \cdot \frac{j j C^{\frac{1}{2}} i j j_{h}^{2}}{\Pi(\Pi i b)}, \text { con } \Pi>b
$$

es decir,

$$
\begin{aligned}
& j j C^{\frac{1}{2}} R(\Pi G) i j j_{h} . \quad \frac{1}{\Pi(\Pi i b)}^{\prod^{\frac{1}{2}}} j j C^{\frac{1}{2}} i j j_{h} \\
& j j C^{\frac{1}{2}} R(\Pi G) i j j h \cdot \frac{1}{\left(\prod_{i} b\right)} j^{j j} C^{\frac{1}{2}} i j j h \text {; }
\end{aligned}
$$

puesto que $D$ es esencia de $C^{\frac{1}{2}}$ también se tiene que

$$
j j C^{\frac{1}{2}} R(\Pi G) i j j_{h} \cdot \frac{1}{\left(\prod i b\right)} j C^{\frac{1}{2}} i j j_{h} \text { para } i 2 D\left(C^{\frac{1}{2}}\right):
$$


Por lo tanto

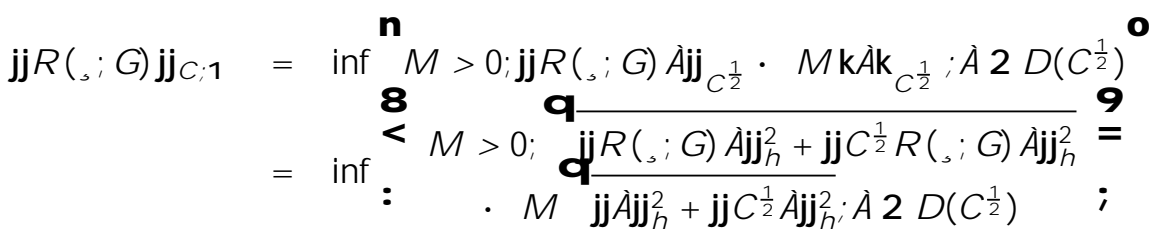

$$
\begin{aligned}
& \text { - } \frac{1}{\Pi i b} \text { : }
\end{aligned}
$$

A hora demostramos la invarianza de $D\left(C^{\frac{1}{2}}\right)$ bajo la acción de $R(\Pi G)$ : Tomemos $i 2 \mathrm{D}$ y sea $\int=\mathrm{R}(\Pi \mathrm{G}) \dot{i}$ : Claramente se tiene que $\int 2 \mathrm{D}\left(\mathrm{C}^{\frac{1}{2}}\right)$, por lo tanto existe $f \int_{n} g_{n, 0}{ }^{1 / 2} D$ tal que $\int_{n} n_{n} !{ }_{1} \int y C^{\frac{1}{2}} \int_{n}{ }_{n !} C^{\frac{1}{2}} \int ; y$

$$
R(\Pi G) \int=\lim _{n ! !} R(\Pi G) \int n
$$

pues $R(\Pi G) \int \mathrm{n} 2 \mathrm{D}\left(\mathrm{C}^{\frac{1}{2}}\right) 8 \mathrm{n}, 0$, lo cual implica que $R(\Pi \mathrm{G}) \int 2 \mathrm{D}\left(\mathrm{C}^{\frac{1}{2}}\right)$, resultando que $R(\Pi G) D\left(C^{\frac{1}{2}}\right) \frac{1}{1 / 2} D\left(C^{\frac{1}{2}}\right)$ :

Corolario 4.1.4 $R(\Pi G)(D)$ es denso en $D\left(C^{\frac{1}{2}}\right)$ con la norma $k: k_{C \frac{1}{2}}$ :

Demostración.

Tomemos $\pi 2 \mathrm{D}\left(\mathrm{C}^{\frac{1}{2}}\right)$ tal que se tenga:

$$
0=h ; R(\Pi G) i^{i}{ }_{C}^{\frac{1}{2}}=h \pi R(\Pi G) i^{i}+{ }^{D} C^{\frac{1}{2}} \pi ; C^{\frac{1}{2}} R(\Pi G) i^{E}
$$

con ¿ 2 D. Vamos a demostrar que $\pi=0$ : Claramente, de la igualdad de arriba se tiene lo siguiente:

$$
\mathrm{D}^{\frac{1}{2}} \pi ; C^{\frac{1}{2}} R(\Pi G) i^{E}=i h ; R(\Pi G) i i
$$

pero recordando que $D$ es denso en $D\left(C^{\frac{1}{2}}\right)$; se tiene que existe $f_{i n} g_{n} 1 / 2 D$ tal que $i_{n} ! n \pi ; C^{\frac{1}{2}} i_{n} !_{n} C^{\frac{1}{2}} \pi ; \mathbb{R}(\Pi G) i_{n} ! \Pi R(\Pi G) \pi, \Pi>0 y$

$C^{\frac{1}{2}} \mathbb{R}(\Pi \mathrm{G}) i_{n} !_{n} C_{D}^{\frac{1}{2}} \mathbb{R}(\Pi \mathrm{G}) \pi$. Por lo tanto se cumple también lo siguiente

$$
C^{\frac{1}{2}} \pi ; C^{\frac{1}{2}} \Pi R(\Pi G) i n^{2}=i m ; \pi(\Pi G) i n_{n} i \quad y
$$

$$
\begin{aligned}
& \mathrm{D}^{\frac{1}{2} \pi ; C^{\frac{1}{2}}} \mathbb{R}(\Pi G) \pi^{E}=\lim _{n ! 1} C^{\frac{1}{2}} \pi ; C^{\frac{1}{2}} R(\Pi G) i n^{E} \\
& =i \lim _{n ! 1} \pi ; \pi(\Pi G) i_{n} i \\
& =i \mathrm{~h} ; \mathbb{R}(\Pi \mathrm{G}) \pi \mathrm{i}:
\end{aligned}
$$


Recordando que $\Pi R(\Pi G) \underset{\Pi ! !_{1}^{s}}{1}$ I, ésta última igualdad nos muestra que

$$
\begin{aligned}
\mathrm{D}_{C^{\frac{1}{2}} \pi ; C^{\frac{1}{2}} \pi} & =i h ; \pi i \\
D_{C^{\frac{1}{2}} \pi ; C^{\frac{1}{2}} \pi}^{E}+h \pi ; \pi i & =0
\end{aligned}
$$

lo cual quiere decir que

$$
\mathrm{jj}_{\mathrm{j} j \mathrm{C}_{\mathrm{C}}^{\frac{1}{2}}}=0
$$

pero esto es posible si y sólo si $\pi=0$ : Por lo tanto resulta que $R(\Pi G)$ (D) es denso en $D\left(C^{\frac{1}{2}}\right)$ :

P roposición 4.1.5 Supongamos que se tiene la Hipótesis $C$. Entonces $D\left(C^{\frac{1}{2}}\right)$ es invariante bajo $\mathrm{P}_{\mathrm{t}}$; para cada $\mathrm{t}$, 0 ; y la restricción de los operadores $\mathrm{P}_{\mathrm{t}}$ a $D\left(C^{\frac{1}{2}}\right)$ forman un semigrupo fuertemente continuo sobre el espacio de Hilbert $\left(D\left(C^{\frac{1}{2}}\right) ; k: k_{C ; 1}\right)$ : Además se tiene que

$$
\mathrm{KP}_{\mathrm{t}} \mathrm{k}_{\mathrm{C} ; 1} \cdot \mathrm{e}^{\mathrm{bt}}
$$

Demostración. Seguimos [8], pág.69.

De la siguiente identidad, válida para cada $\Pi \pi>b$

$$
R(\Pi G) \text { i } R(\pi ; G)=(\pi ; \Pi R(\Pi G) R(\pi ; G) ;
$$

se obtiene una familia de resolventes en el espacio deHilbert $\left(D\left(C^{\frac{1}{2}}\right) ; k^{\prime}: k_{C ; 1}\right)$; $y \operatorname{ker}(R(\Pi G))=$ fog; pues $R(\Pi G)(\Pi G)=1$ implica que $R(\Pi G)$ es inyectiva, por el corolario de arriba, ahora usando [20] Teorema 9;3 pág.36, se tiene que $f R(\Pi G) g_{\Pi>b}$ es el resolvente de un operador cerrado y densamente de.nido en $\mathrm{D}\left(\mathrm{C}^{\frac{1}{2}}\right)$; llamémosle a : Por el Teorema de Hille-Yosida-P hillips-M iyadera-Feller.([17]; pág;72) y la proposición anterior se tiene que ${ }^{a}$ genera un semigrupo de contracciones $\mathrm{fS}_{\mathrm{t}} \mathrm{g}_{\mathrm{t}, 0}$ de clase $\mathrm{C}_{0}(1 ; \mathrm{b}) ; \mathrm{es}$ decir $\mathrm{jjS}_{\mathrm{t}} \mathrm{jj} \mathrm{c} ; 1 \cdot \mathrm{e}^{\mathrm{bt}}$; y como

$$
\mathrm{S}_{\mathrm{t}}=\lim _{\mathrm{n} !}^{3} \frac{\mathrm{n}}{\mathrm{t}}^{3} \mathrm{R}^{3} \frac{\mathrm{n}}{\mathrm{t}} ; \mathrm{G}^{\prime \prime}
$$

fuertemente en $D\left(C^{\frac{1}{2}}\right)$ entonces para todo $\int 2 D\left(C^{\frac{1}{2}}\right)$ ([19];Teorema 8;3; pág;33)

$$
\begin{gathered}
S_{t} \int=\lim _{n !} m_{1}^{3} \frac{n^{3}}{t} R^{3} \frac{n}{t} ; G^{\prime}{ }^{n} \int=P_{t} \int \\
\underline{a} \int=\lim _{t !} \frac{S_{t} \int i \int}{t}=1 \prod_{0^{+}} \frac{P_{t} \int i \int}{t}=G \int ;
\end{gathered}
$$

resultando así que a es la parte de $\mathrm{G}$ en $\mathrm{D}\left(\mathrm{C}^{\frac{1}{2}}\right)$ ([19];Def;10;3;pág;39):

Por lo tanto $P_{t}$ deja invariante a $D\left(C^{\frac{1}{2}}\right)$; resultando a la vez que es semigrupo, y además $j \mathrm{j} P_{\mathrm{t}} \mathrm{jj} \mathrm{c} ; 1 \cdot \mathrm{e}^{\mathrm{bt}}$. 
Corolario 4.1.6 Supongamos que tenemos la Hipótesis C. E ntonces para cada i $2 R(\Pi G)(D)$ con $\Pi>b$, la función $t ! j j C^{\frac{1}{2}} P_{t} i j j$ h es diferenciable y además:

$$
\frac{d}{d t} j j C^{\frac{1}{2}} P_{t i} j j_{h}^{2}=2 R e^{D} C^{\frac{1}{2}} P_{t} i C^{\frac{1}{2}} G P_{t i}{ }^{E}:
$$

Demostración. Seguimos [8], pág.69.

De la Teoría de semigrupos de contracciones, aplicada a

$$
\mathrm{P}_{\mathrm{t}}: \mathrm{D}^{3} \mathrm{C}^{\frac{1}{2}} ! \mathrm{D}^{3} \mathrm{C}^{\frac{1}{2}}
$$

se sigue que $R(\Pi G)$ D $C^{\frac{1}{2}}=D(\underline{a})$ y además para $<2$ D ( $(\underline{a})$

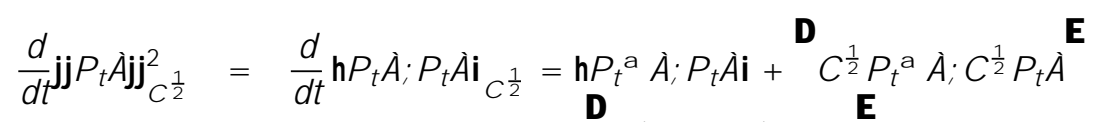

$$
\begin{aligned}
& +h P_{t} i P_{t}{ }^{a}{ }^{a}{ }_{C}{ }^{\frac{1}{2}}+C^{\frac{1}{2}} P_{t} i ; C^{\frac{1}{2}} P_{t}{ }^{a} i \\
& =\frac{d}{d t} j j P_{t} i j j j_{h}^{2}+2 R e^{D} C^{\frac{1}{2}} P_{t} i ; C^{\frac{1}{2}} P_{t}{ }^{\underline{a}} i^{E}
\end{aligned}
$$

Por otro lado como $\stackrel{a}{i=G_{i}}$ para $i 2 \mathrm{D}(\underline{\mathrm{a}})$

$$
\begin{aligned}
j j C^{\frac{1}{2}} P_{t i j j} j_{h}^{2} & =j j P_{t i j j}{ }_{C \frac{1}{2}} i j j P_{t} i j j_{h}^{2} \\
\frac{d}{d t} j C^{\frac{1}{2}} P_{t i j j j_{h}^{2}} & =\frac{d}{d t} j P_{t} i j j_{C}^{2}{ }_{C}^{\frac{1}{2}} i \frac{d}{d t} j j P_{t} i j j_{h}^{2} \\
& =2 R e C^{\frac{1}{2}} P_{t i} ; C^{\frac{1}{2}} P_{t}{ }^{a}{ }^{E}
\end{aligned}
$$

por lo tanto se tiene que

$$
\frac{d}{d t} j j C^{\frac{1}{2}} P_{t} i j j_{h}^{2}=2 R e^{D} C^{\frac{1}{2}} P_{t} i ; C^{\frac{1}{2}} P_{t} \frac{a}{i} i^{E}:
$$

Proposición 4.1.7 Supongamos que tenemos la Hipótesis C. Entonces para cada $\Pi>b$ y $<2 \mathrm{D}\left(\mathrm{C}^{\frac{1}{2}}\right)$ se tiene lo siguiente:

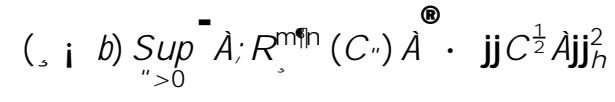

donde

$$
C_{1 "}=\left(1+{ }^{n} \mathrm{C}\right)^{i}{ }^{1} \mathrm{C}:
$$

Demostración. Seguimos [8], pág.70.

Sea $f R{ }_{n}^{n} g_{n>0}$ la sucesión de transformaciones lineales positivas consideradas en el Teorema 3;2;4. Será su..ciente demostrar que para todo $n, 0 ; \Pi>$ by 
¿ $2 \mathrm{D}\left(\mathrm{C}^{\frac{1}{2}}\right)$ el operador $R_{\Pi}^{n}\left(C_{11}\right)$ satisface:

(Пi b) $\operatorname{Suph}_{\text {" }>0} ; R_{\Pi}^{n}\left(C_{u}\right) i i \cdot j j C^{\frac{1}{2}} i j j{ }_{h}^{2}:$

Veamos que la desigualdad se vale para $n=0$ y $~<2 R(\Pi G)(D)$ : Procedemos por inducción. Del hecho de que $D$ es invariante bajo $P_{t}$ y que $P_{t}$ conmuta con $R(\Pi G)$ se tiene lo siguiente

$$
P_{t} R(\Pi G)(D)=R(\Pi G) P_{t}(D) \mu R(\Pi G)(D):
$$

Con métodos de Teoría espectral se tiene que $h_{i} ; C_{1 " i} i \cdot h_{i} ; C_{i} i$ para todo ¿ 2 D (C) ; y como D (C) es denso en $C^{\frac{1}{2}}$ ([15]; Teorema 3;35; pág. 281) también se tiene que $h_{i} ; C_{1} i \mathrm{i}$. $C^{\frac{1}{2}} i ; C^{\frac{1}{2}} i$ : A hora integrando por partes tenemos:

$$
\begin{aligned}
& \Pi^{-} i ; R_{\Pi}^{0}\left(C_{11}\right) i^{\circledR}=\Pi_{Z^{0}}^{Z_{1}} \text { e } \Pi^{t} h P_{t} i ; C_{n} P_{t} i i d t
\end{aligned}
$$

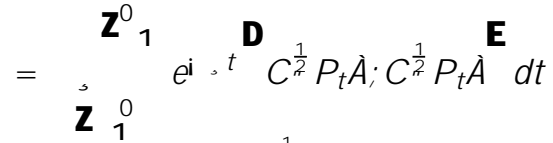

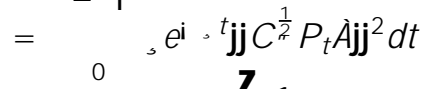

$$
\begin{aligned}
& =j j C^{\frac{1}{2}} i j j^{2}+Z_{\theta} 2 R e e^{i t} C^{C_{12}^{\frac{1}{2}}} P_{t} i ; C_{1}^{\frac{1}{2}} G P_{t} i^{E} d t \\
& \text { - } \quad j j C^{\frac{1}{2}} i j j h_{h}^{2}+b^{1} e^{i} \Pi_{j j} j C^{\frac{1}{2}} P_{t} i j j^{2} d t \\
& \text { - } \quad j j C^{\frac{1}{2}} i j j_{h}^{2}+b^{-} i ; R_{\Pi}^{0}\left(C_{11}\right) i^{\circledR} \\
& \text {. } \quad j j C^{\frac{1}{2}} i j j_{h}^{2}+\operatorname{bSup}_{n>0} i ; R_{\Pi}^{0}\left(C_{11}\right) i \stackrel{\circledR}{:}
\end{aligned}
$$

Así se tiene que,

$$
\prod_{n \rightarrow 0}{ }^{-} i ; R_{\Pi}^{0}\left(C_{n}\right) i^{\circledR} \cdot j j C^{\frac{1}{2}} i j j_{h}^{2}+\operatorname{bSup}_{n>0}^{-} i ; R_{\Pi}^{0}\left(C_{n}\right) i^{\circledR}
$$

lo cual implica que,

$$
\left(\Pi_{i} \quad \text { b) } \operatorname{Sup}_{n>0}^{-} i ; R_{\Pi}^{0}\left(C_{n}\right) i^{\circledR} \cdot j j C^{\frac{1}{2}} i j j_{h}^{2}:\right.
$$

Por la densidad de $R(\Pi G)(D)$ en $D\left(C^{\frac{1}{2}}\right)$ se extiende el resultado en $D\left(C^{\frac{1}{2}}\right)$ : Supongamos que la proposición se tiene para algún $n$ y para cada i 2 $D\left(C^{\frac{1}{2}}\right)$ : Es fácil ver que para cada $i 2 R(\Pi G)(D), L \cdot P_{t} i 2 D\left(C^{\frac{1}{2}}\right)$ de donde por la segunda ecuación de $(3 ; 9)$, y de la de.nición de $R_{\Pi}^{n}$ del Teo- 
rema 3;2;4; se tiene que:

$$
\begin{aligned}
& { }^{2} ; R_{\Pi}^{n+1}\left(C^{\prime \prime}\right) i^{\circledR}=h_{i} ; F_{\Pi}\left(C^{\prime \prime}\right) i \mathrm{i} \\
& x Z_{1} \\
& +\quad e^{i} \Pi^{t} h L \cdot P_{t} i ; R_{\Pi}^{n}\left(C_{11}\right) L \cdot P_{t} i i d t
\end{aligned}
$$

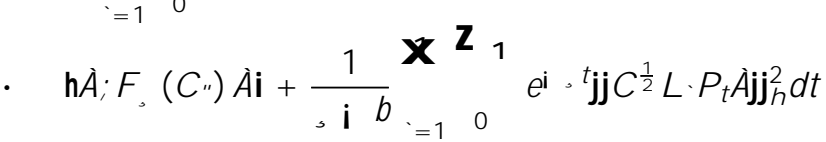

ahora usando la desigualdad 4;1) e integrando por partes nuevamente se tiene que

$$
\begin{aligned}
& \times Z_{1}
\end{aligned}
$$

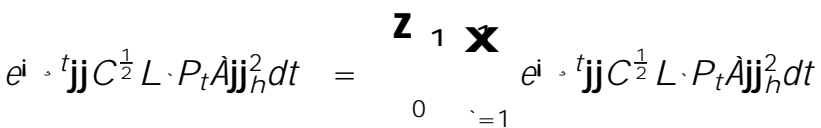

$$
\begin{aligned}
& \checkmark=1 \quad 0 \\
& =Z_{Z_{1}^{0} X^{\prime}=1}^{Z_{1}} e^{i t^{t}} C^{\frac{1}{2}} L \cdot P_{t} i ; C^{\frac{1}{2}} L \cdot P_{t} i^{E} d t \\
& \text { - } \quad \text { bei } \Pi t_{j j} C^{\frac{1}{2}} P_{t} i j j_{h}^{2} d t \\
& i_{Z_{1}}^{{ }^{0} Z_{1}} e^{i \frac{}{t} 2 R e ~} C^{\frac{1}{2}} P_{t} i ; C^{\frac{1}{2}} G P_{t} i^{E} d t
\end{aligned}
$$

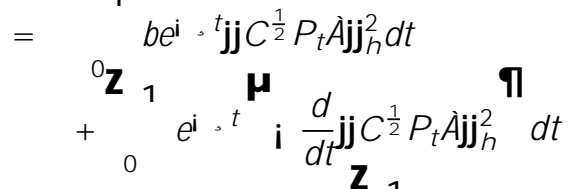

$$
\begin{aligned}
& =j j C^{\frac{1}{2}} i j j_{h}^{2} i(\Pi i \quad b){ }_{0}^{1} e^{i{ }^{i} j j C^{\frac{1}{2}} P_{t} i j j_{h}^{2} d t} \\
& \text { - } \quad j j C^{\frac{1}{2}} i j j_{h}^{2} i\left(\Pi_{i} \quad b\right) h_{i} ; F_{\Pi}\left(C_{n}\right) i i
\end{aligned}
$$

por lo que se deduce que,

$$
-i ; R_{\Pi}^{n+1}\left(C_{11}\right) i^{\circledR} \cdot h_{i} ; F_{\Pi}\left(C_{11}\right) i i+\frac{j j C^{\frac{1}{2}} i j j j_{h}^{2}}{\prod_{i} b} i h_{i} ; F_{\Pi}\left(C_{11}\right) i i
$$

con lo cual

$$
\text { (Пi b) } \operatorname{Sup}^{-} i ; R_{\Pi}^{n+1}\left(C_{1 "}\right) i^{\circledR} \cdot j j C^{\frac{1}{2}} i j j_{h}^{2}
$$

y por ser $R(\Pi G)(D)$ denso en $D\left(C^{\frac{1}{2}}\right)$; la prueba vale en todo $D\left(C^{\frac{1}{2}}\right)$ :

Los siguientes tres resultados son los más importantes de este capítulo y son consecuencias de la Hipótesis C. 
Teorema 4.1.8 Supongamos que hay un operador $\mathrm{C}$ que satisface la $\mathrm{H}$ ipótesis C, la Hipótesis A A y tal que

$$
h_{i} ; J_{n i} \cdot h_{i} ; C_{i} i
$$

para cada i $2 \mathrm{D}(\mathrm{C}) ; \mathrm{n}, 1$ :

Entonces el semigrupo dinámico cuántico minimal es conservativo.

Demostración. Seguimos [8], pág. 72.

Sea $\Pi>$ b;bajo las hipótesis del mismo teorema tenemos que los operado-

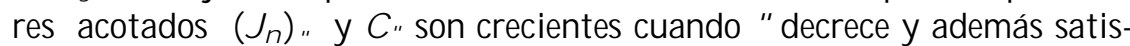
facen la desigualdad

$$
\left(\mathrm{J}_{\mathrm{n}}\right) " \cdot \mathrm{C}^{\prime \prime}
$$

([16]; pág;317) con lo cual para ¿ $2 \mathrm{D}(\mathrm{C})$ se tiene que:

$$
\begin{aligned}
& -i ; R_{\Pi}^{\operatorname{mmn}}\left(J_{n}\right)_{11} i^{\circledR} \cdot-i ; R_{\Pi}^{\operatorname{mm}}\left(C_{11}\right){ }^{\circledR}
\end{aligned}
$$

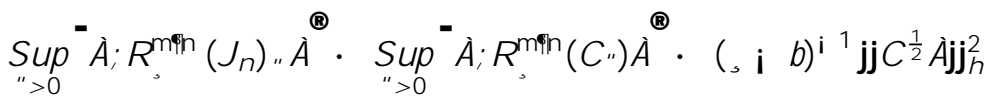

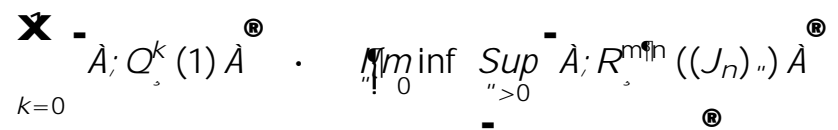

$$
\begin{aligned}
& =\lim _{n} \operatorname{minf}{ }^{-} i ; R_{\Pi}^{\min }\left(J_{n}\right) i^{\circledR}<1
\end{aligned}
$$

lo que implica que

$$
\lim _{k !} \lim _{1}^{-} i ; Q_{\Pi}^{k}(1) i \stackrel{\circledR}{\circledR}=0
$$

y como jjQ $\mathrm{Q}_{\Pi}^{\mathrm{k}}(1) \mathrm{jj}_{1} \cdot$ · 1 , por lo tanto tenemos que $\mathrm{Q}_{\Pi}^{\mathrm{k}}(1){ }_{\mathrm{k}} !^{\mathrm{w}}{ }_{1}$ 0; resultando así que $T^{m}$ es conservativo. Siendo $D(C)$ esencia de $C^{\frac{1}{2}}$ ( [14] Teorema 2;1, pág;322;T eorema 2;23,pág.331) la demostración se extiende a todo $\mathrm{D}\left(\mathrm{C}^{\frac{1}{2}}\right)$.

Teorema 4.1.9 Ahora supongamos que tenemos la Hipótesis A A y que existe un operador positivo autoadjunto $\mathrm{i}$ en $\mathrm{h}$ tal que :

1) $D(G) 1 / 2 D\left(i^{\frac{1}{2}}\right)$ y que para cada $i 2 D(G)$ se tiene lo siguiente:

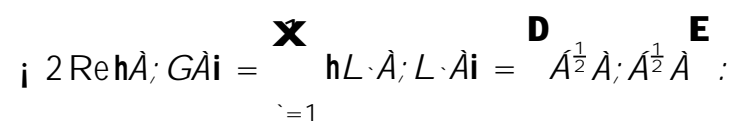

2) Existe un operador positivo autoadjunto $C$ satisfaciendo la Hipótesis $C$ 
tal que $D(C) \frac{1}{1 / 2} D\left(i^{\frac{1}{2}}\right)$ y para cada $i 2 D(C)$ se tiene que

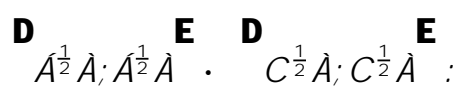

Entonces el semigrupo dinámico cuántico minimal $\mathrm{T}^{\text {min }}$ es conservativo.

Demostración. Seguimos [8], pág.72.

Sea $\Pi>$ by i 2 D (i ): Para " > 0, los operadores acotados i " y C " satisfacen

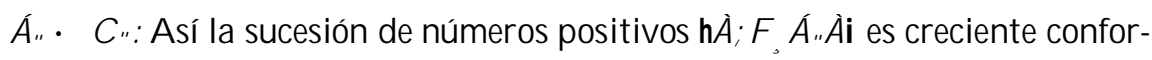
me " decrece, veamos que los $F_{\Pi i}$ " están acotados uniformemente. Para ello sea $i 2 D(G) \frac{1}{2} 2 D\left(i^{\frac{1}{2}}\right)$ : Obtenemos que $P_{s i} 2 D(G) \frac{1}{2} D\left(i^{\frac{1}{2}}\right)$ y por lo tanto

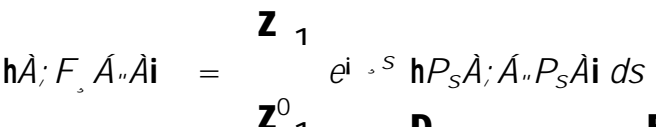

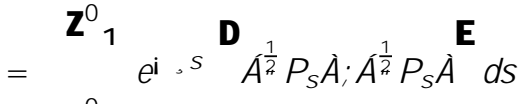

$$
\begin{aligned}
& Z_{1}^{0} \text { ei } \Pi s^{D} i^{\frac{1}{2}} P_{s i} ; i^{\frac{1}{2}} P_{s i} E d s \\
& Z_{1}^{0} \\
& =Z_{Z_{1}^{0}} \mathrm{e}^{\mathrm{i} \Pi \mathrm{s}}\left(\mathrm{i} 2 \mathrm{RehP_{s } i ; G P _ { s } i \mathrm { i } ) d s}\right. \\
& ={ }_{0}^{1} e^{i \Pi s} i \frac{d}{d s} j j P_{s} i j j h h
\end{aligned}
$$

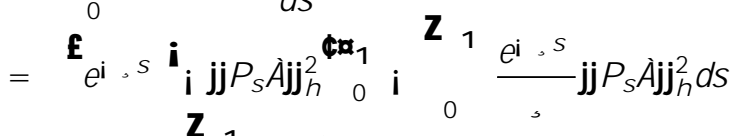

$$
\begin{aligned}
& =j j i j j h_{h}^{2} i_{0}^{Z_{1}} \frac{e^{i} \Pi s}{\Pi} j j P_{s i j j} j_{h}^{2} d s \\
& \text { - } \quad \mathrm{jj}_{\mathrm{j} j \mathrm{jj}}^{2} \text { : }
\end{aligned}
$$

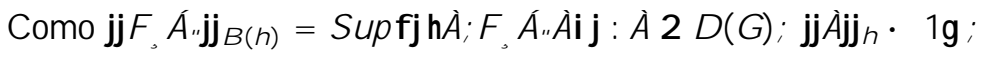
se tiene que $j j F_{\Pi i}{~ " j j_{B}(h)}$ 1; lo cual implica que $F_{\Pi i}$ " está uniformemente acotado por 1: A hora haciendo uso del Teorema de convergencia monó- 
tona tenemos

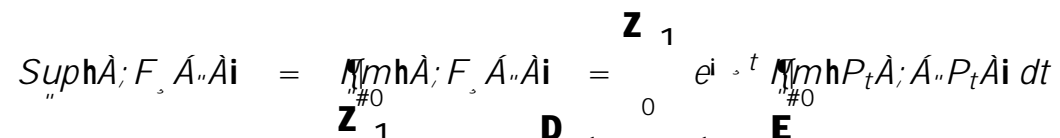

$$
\begin{aligned}
& =Z_{Z_{1}^{0}}^{Z^{1} e^{i t} \lim _{1 \neq 0} i^{\frac{1}{12}} P_{t} i ; i^{\frac{1}{2}} P_{t} i} d t \\
& =e^{i \Gamma t} j j i^{\frac{1}{2}} P_{t} i j j^{2} d t \\
& =Z_{0}^{Z_{1}} \mathrm{e}^{\mathrm{i} \Pi \mathrm{t}}{ }_{=1}^{X} \mathrm{~L} \cdot \mathrm{P}_{\mathrm{t} i} ; L \cdot P_{\mathrm{t}} i \mathrm{i} d \mathrm{t} \\
& =x^{A Z_{1}} e^{i{ }^{\pi t}} \mathrm{~L} \cdot P_{t} i ; L \cdot P_{t} i \mathrm{dt} \\
& =1 Z_{1}^{0}
\end{aligned}
$$

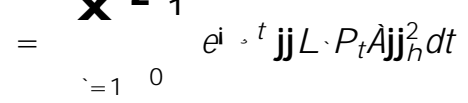

$$
\begin{aligned}
& =h_{i} ; Q_{\Pi}(1) i \mathrm{i}
\end{aligned}
$$

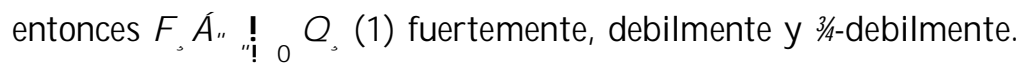
Por otra parte recordemos que los $Q_{\Pi}^{k}$ son æedébiles continuos. Entonces

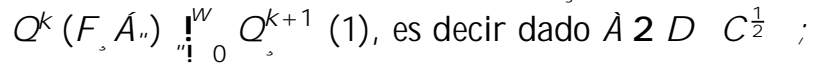

$$
-i ; Q^{k}\left(F_{\Pi i n}\right) i^{\circledR}{ }_{n !}^{\circledR}{ }^{-} i ; Q_{\Pi}^{k+1}(1) i \stackrel{\circledR}{~:}
$$

A hora tenemos

$$
\begin{aligned}
& { }_{k=0}^{X}{ }^{A} ; Q_{\Pi}^{k+1}(1) i^{\circledR}={ }_{k=0}^{X} \lim _{n \neq 0}{ }^{-} i ; Q^{k}\left(F_{\Pi i ~}\right) i^{\circledR} \\
& =\lim _{n \neq 0} \sum_{k=0}^{A}-i ; Q^{k}\left(F_{\Pi i n}\right) i^{\circledR} \\
& =\operatorname{Sup}_{n>0}{ }^{k=0} i ; R_{\Pi}^{\operatorname{mm}}\left(i \mu^{\prime \prime}\right) i^{\circledR} \\
& \text { - } \operatorname{Sup}_{\text {">0 }} i ; R_{\Pi}^{\operatorname{mm}}\left(C_{11}\right) i^{\circledR} \\
& \text { - } \frac{1}{\Pi i b} j C^{\frac{1}{2}} i j j_{h}^{2} \text {; }
\end{aligned}
$$

así se tiene que ${ }^{-} i ; Q_{\Pi}^{k+1}(1) i_{k !}^{\circledR}{ }_{1} !{ }_{1}$ : Como $D\left(C^{\frac{1}{2}}\right)$ es denso en $h$ tenemos que $Q_{\Pi}^{k+1}(1){ }_{k !}{ }_{1} 0$ y por lo tanto $T^{m m}$ es conservativo. 
Proposición 4.1.10 Supongamos que se tiene la Hipótesis $A A$ y que existe un operador autoadjunto $C$ con dominio coincidiendo con el de $G$. Sea $D_{1}$ una esencia para $C$ con las siguientes propiedades:

1) L. $\left(D_{1}\right) \mu D\left(C^{\frac{1}{2}}\right)$ para ` , 1 :

2) Existe un operador autoadjunto i tal que $D_{1} 1 / 2 D(i)$ y además

$$
\text { i } 2 R_{e} h_{i} ; G_{i} i=h_{i} ; i \dot{i} \cdot h_{i} ; C_{i} i
$$

para todo $i 2 \mathrm{D}_{1:}$

3) Existe una constante positiva b tal que la desigualdad

$$
2 \operatorname{RehC}_{i} ; \mathrm{G}_{i} i+{ }_{=1}^{\mathrm{A}} \mathrm{C}^{\frac{1}{2}} \mathrm{~L} \cdot \dot{i} ; \mathrm{C}^{\frac{1}{2}} \mathrm{~L} \cdot \dot{\mathrm{E}} \cdot \mathrm{bh}_{i} ; \mathrm{C}_{i} i
$$

se tiene para todo $i 2 \mathrm{D}_{1}$ :

Entonces el semigrupo dinámico cuántico minimal es conservativo.

Demostración. Seguimos [8], pág.74.

Consideremos $D(G)$ y $D(C)$ como espacios de Hilbert con sus respectivas normas

$$
\begin{aligned}
& j j u j j_{G}=q \\
& j j u j j_{C}=q \frac{j j u j j_{h}^{2}+j j G u j j_{h}^{2}}{j j u j j_{h}^{2}+j j C u j j_{h}^{2}}:
\end{aligned}
$$

Con éstas dos normas se tiene que la identidad $i: D(G) ! D(C)$; es una transformación cerrada y además es un homeomor..smo. R esultando así que las dos normas son equivalentes y por lo tanto existen constantes $C_{1}$; $\mathrm{C}_{2} ; \mathrm{C}_{3} ; \mathrm{C}_{4}$ tales que:

$$
\begin{array}{ll}
j j G_{i j j h} & \cdot \quad c_{1} j j C_{i j j} j_{h}+c_{2} j j i j j_{h} \\
j j C_{i j j_{h}} \cdot & c_{3} j j G_{i j j} j_{h}+c_{4} j j i j j_{h},
\end{array}
$$

ya que $D_{1}$ es una esencia de $C$ y de $G$; para cada $i 2 D(C)=D(G)$ existe $f_{i n} g_{n, 1} 1 / 2 D_{1}$ tal que $i_{n} !{ }_{n}$ i y $f_{i n} g_{n, 1}, f C_{i n} g_{n, 1}$ convergen fuertemente a $G_{i}$ y a $C_{i}$ : Vamos a demostrar que se cumple la condición 1) del Teorema 4;1;9; recordemos que en esta hipótesis se requiere que $D(G) \frac{1}{2} D\left(i^{\frac{1}{2}}\right)$ y para todo $i 2 D(G)$

$$
\text { i } 2 \text { Reh } i G_{i} i={ }_{=1}^{A} H \cdot i ; L \cdot i i=i^{\frac{1}{2}} i ; i^{\frac{1}{2}} i \text { : }
$$

Sea $i 2 D(G)$, por demostrar que $i 2 D\left(i^{\frac{1}{2}}\right)$ :

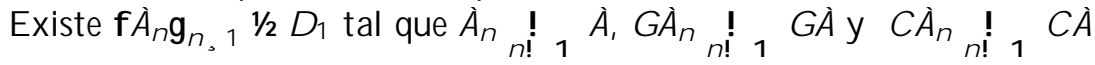


así por la Hipótesis 2)

$$
\begin{aligned}
& \text { i } 2 \text { Reh } h_{n} ; G_{i n} i=h_{i n} ; i i_{n} i \\
& \text { i } 2 \text { Reh } ; G_{i} i=\lim _{n !} h_{i n} ; i i_{n} i \\
& \text { y como } h_{i n} ; i i_{n} i=i^{\frac{1}{2}} \dot{L}_{n} ; i^{\frac{1}{2}} \dot{L}_{n}=j j^{\frac{1}{2}} i_{n} j j^{2} \text {; ahora resta demostrar que } \\
& i^{\frac{1}{2}} \sum_{n} \text { es convergente. Obsérvese lo siguiente: }
\end{aligned}
$$

$$
\begin{aligned}
& j \mathrm{ji} \mathrm{i}^{\frac{1}{2}}\left(i_{n} \mathrm{i} i_{\mathrm{m}}\right) \mathrm{jj}^{2}=\mathrm{i}^{\frac{1}{2}}\left(i_{n} \mathrm{i} i_{\mathrm{m}}\right) ; \mathrm{i}^{\frac{1}{2}}\left(i_{n} \mathrm{i} i_{\mathrm{m}}\right)^{\mathrm{E}} \\
& \left.=H_{i n} \mathrm{i} i m\right) ; \mathrm{i}\left(i_{n} \mathrm{i} i_{m}\right) \mathrm{i} \\
& \text { - } \quad\left(i_{n} \text { i } i_{m}\right) ; C\left(i_{n} \text { i } i_{m}\right) \mathrm{i} \\
& \text { - jjin i } i_{m} j j_{h} j j C\left(i_{n} \mathrm{i} \quad i_{m}\right) j_{j} j_{n ; m ! ~} 0 \text {; }
\end{aligned}
$$

por lo tanto ${ }^{n} i^{\frac{1}{2}} \sum_{n}{ }_{n, 1}$ es una sucesión de Cauchy y en consecuencia es convergente.

Supongamos que $i^{\frac{1}{2}} i_{n}{ }_{n} !{ }_{1} \int y i_{n}{ }_{n}{ }_{1} i$ : Como $i=i^{\text {a }}$ entonces es cerrado y se tiene que $i 2 D\left(i^{\frac{1}{2}}\right)$ y $i^{\frac{1}{2}} i=\int$. Con lo cual $D(G) \frac{1 / 2}{D}\left(i^{\frac{1}{2}}\right)$;

deduciéndose también que,

$$
\begin{aligned}
& \text { i } 2 \text { Reh } ; G_{i} i=\lim _{n ! l}\left(i 2 \operatorname{Reh}_{i n} ; G_{E} i\right) \\
& =\lim _{B^{\prime}} i^{\frac{1}{2}} \sum_{n} ; i^{\frac{1}{2}} i_{n} \\
& =i^{\frac{1}{2}} i ; i^{\frac{1}{2}} i^{E}
\end{aligned}
$$

para todo ¿ $2 \mathrm{D}(\mathrm{G})$ : A sí hemos probado que se cumple la Condición 1) del Teorema 4;1;9:

A hora como $D(C) \frac{1}{2} D\left(i^{\frac{1}{2}}\right)$ ya que $D(C)=D(G)$; sea $i 2 D(G)$ entonces existe f $i_{n} g_{n, 1} 1 / 2 D_{1}$ tal que $i_{n} !{ }_{n}{ }_{1}, G_{i n} !{ }_{n} G_{i}, C_{i n}{ }_{n !} C_{i} y$ $i^{\frac{1}{2}} \sum_{n} ! i_{1} i^{\frac{1}{2}} i$. Se tiene entonces que

$$
\begin{aligned}
& i^{\frac{1}{2}} i ; i^{\frac{1}{2}} i^{E}=\lim _{n !} i^{\frac{1}{2}} i_{n} ; i^{\frac{1}{2}} i_{n} \\
& \text { - } \quad \lim _{n !} h_{i} ; C_{i n} i=h_{i} ; C_{i} i=C^{\frac{1}{2}} i ; C^{\frac{1}{2}} i^{E}
\end{aligned}
$$

lo cual quiere decir que

$$
i^{\frac{1}{2}} i ; i^{\frac{1}{2}} i^{E} \cdot C^{\frac{1}{2}} i ; C^{\frac{1}{2}} i^{E} \text { : }
$$


Para ..nalizar la demostración de éste teorema veamos que se satisfacen las condiciones de la Hipótesis C:

1) Tomando $D=D(G)$ se cumple la primera condición.

2) D (G) es esencia de $C^{\frac{1}{2}}$ ([15],Teorema 2;1; pág. 322 y Teorema 2;23; pág. 331).

3) Siempre se cumple.

4) Para veri..car, $L \cdot R(\Pi G)(D(G)) \frac{1}{1 / 2} D\left(C^{\frac{1}{2}}\right)$ : Veamos que $R(\Pi G)(D(G))$ $1 / 2 D(L \cdot)$ y que $L \cdot$ ¿ $2 \mathrm{D}\left(\mathrm{C}^{\frac{1}{2}}\right)$, para ¿ $2 \mathrm{R}(\Pi \mathrm{G})(\mathrm{D}(\mathrm{G}))$ :

Por hipótesis tenemos,

$$
D(G) 1 / 2 \backslash \stackrel{1}{=} D(L \cdot)
$$

lo cual implica que

$$
R(\Pi G)(D(G)) \frac{1}{1} 2 D(G) \frac{1}{2} D(L \cdot) \text { para todo ` } 1:
$$

A sí, sólo falta veri..car que $L$ ¿ $2 \mathrm{D}\left(C^{\frac{1}{2}}\right)$ : P or otra parte existe $f_{i n} g_{n}, 1 \frac{1 / 2}{2}$ $D_{1}$ tal que $i_{n} !{ }_{n} i_{1}, G_{i n} ! G_{i}, C_{i n} ! C_{n} C_{i} y^{\frac{1}{2}} i_{n} !{ }_{1} i^{\frac{1}{2}} i$. Gracias a la ecuación de la Hipótesis $A \mathrm{~A}$ ) también se tiene que:

$$
\begin{aligned}
0 & <\mathrm{L} \cdot\left(i \mathrm{i} i_{n}\right) ; \mathrm{L} \cdot\left(i \mathrm{i} i_{n}\right) \mathrm{i} \cdot{ }^{X} \mathrm{H} \cdot\left(i \mathrm{i} i_{n}\right) ; \mathrm{L} \cdot\left(i \mathrm{i} i_{n}\right) \mathrm{i} \\
& =\mathrm{i} 2 \operatorname{Reh}\left(i \mathrm{i} i_{n}\right) ; G\left(i \mathrm{i} i_{n}\right) \mathrm{i} n ! 10
\end{aligned}
$$

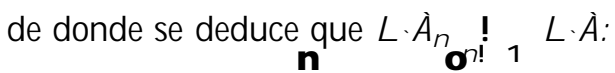

A hora veamos que $C^{\frac{1}{2}} L \cdot \dot{i}_{n} n_{n, 1}$ es sucesión de Cauchy

$$
\begin{aligned}
& j j C^{\frac{1}{2}} L \cdot i_{n} \text { i } C^{\frac{1}{2}} L \cdot i m j j_{h} \cdot{ }_{=1}^{D} C^{\frac{1}{2}} L \cdot\left(i_{n} \text { i } i_{m}\right) ; C^{\frac{1}{2}} L \cdot\left(i_{n} \text { i } i_{m}\right)^{E} \\
& \text { - } \quad b h\left(i_{n} \text { i } i_{m}\right) ; C\left(i_{n} \text { i } i_{m}\right) \text { i i } 2 \operatorname{RehC}\left(i_{n} \text { i } i_{m}\right) ; G\left(i_{n} \text { i } i m_{m}\right) \text { i } \underset{n ; m ! 1}{ } 0 \text { : }
\end{aligned}
$$

Sea $\int=\lim _{n ! m_{1}} C^{\frac{1}{2}} L \cdot i_{n} ;$;omo $C^{\frac{1}{2}}$ es cerrado entonces $L$ ¿ ¿ $2 D\left(C^{\frac{1}{2}}\right)$ y $C^{\frac{1}{2}} L \cdot \mathcal{i}=\lim _{n !} C_{1}^{\frac{1}{2}} L \cdot i_{n}:$ 
Para ver que se cumple la parte 5) de la Hipótesis C , tomemos \& $2 \mathrm{R}(\Pi \mathrm{G})(\mathrm{D}(\mathrm{G})) \frac{1}{1 / 2} \mathrm{D}(\mathrm{C})^{1 / 2} \mathrm{D} \quad \mathrm{C}^{\frac{1}{2}}$, obsérvese lo siguiente

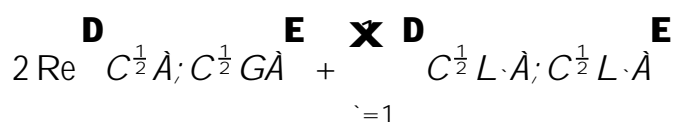

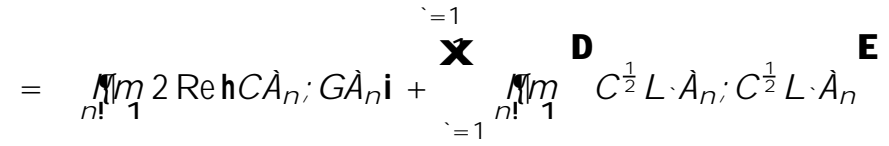

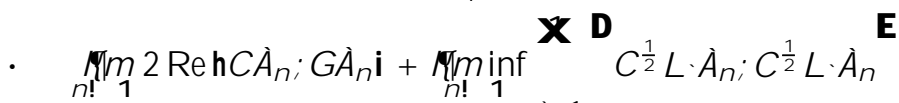

$$
\begin{aligned}
& \tilde{A} \quad \begin{array}{ccc}
n ! 1 & =1 \\
D & E^{1}
\end{array} \\
& =\liminf _{n ! 1} 2 R e h C i_{n} ; G_{i n} i+{ }_{=1}^{*} C^{\frac{1}{2}} L \cdot i_{n} ; C^{\frac{1}{2}} L \cdot i_{n} \\
& \text { - } \quad \min _{n !} \operatorname{infbh}_{i} ; C_{i_{n}} \mathrm{i} \\
& =\liminf _{n !} C_{D} C^{\frac{1}{2}} i_{n} ; C^{\frac{1}{2}} i_{E} E \\
& =\lim _{n ! D^{1}} b C^{\frac{1}{2}} i n ; C^{\frac{1}{2}} i n \\
& =b C^{\frac{1}{2}} i_{n} ; C^{\frac{1}{2}} i_{n}<1 \text { : }
\end{aligned}
$$

Por lo tanto $C$ satisface la Hipótesis $C$, y por el teorema anterior $\mathrm{T}^{\text {min }}$ es conservativo. 


\section{Capítulo 5}

\section{Ejemplos de S. D. C.}

A qui se exponen algunos ejemplos en los que el correspondiente semigrupo dinámico cuántico minimal asociado resulta ser conservativo en algunos casos, un ejemplo en el cual el minimal es conservativo es el 5;1;4 y otro en donde no lo es, es el ejemplo 5;1;6 con $\varnothing^{\alpha}<\varnothing$. Este último ejemplo muestra que la condición A A es necesaria pero no su..ciente para la conservatividad. 


\subsection{Ejemplos}

Ejemplo 5.1.1 ([8]; pág. 30:)Sea h cualquier espacio de Hilbert complejo separable, y sea $P=f P_{t} g_{t}$ o un semigrupo uniformemente continuo de contracciones en $h$. La familia de operadores $T_{t}: B(h) ! B(h)$ de..nidos por

$$
T_{t}(a)=P_{t}^{\alpha} a P_{t}
$$

es un Semigrupo Dinámico Cuántico uniformemente continuo, cuyo generador in..nitesimal es $\$(\mathrm{a})=\mathrm{G}^{\mathrm{\alpha}} \mathrm{a}+\mathrm{aG}$ donde $\mathrm{G}$ es el generador de $\mathrm{P}$ :

Veamos que $T_{t}$ es un SDC.

1) $T_{0}(a)=P_{0}^{x} a P_{0}=a$

2) $T_{t+s}(a)=P_{t+s}^{a} a P_{t+s}=P_{t}^{a}\left(P_{s}^{a} a P_{s}\right) P_{t}=P_{t}^{a}\left(T_{s} a\right) P_{t}=T_{t}\left(\left(T_{s} a\right)\right)$

$=\left(T_{t} T_{s}\right)(a)$

3) Por $\mathrm{K}$ raus;Capítulo 1: Teorema 1;2;15, $\mathrm{T}_{\mathrm{t}}$ resulta ædébil continuo.

4) Ahora veamos que es ædébil continuo en el tiempo t; para ello tomemos

$\Omega 2 \varnothing(\mathrm{h})$ tal que

$$
\Omega={ }_{n=1}^{X} F_{h} j e_{n} i h_{n} j
$$

donde $\mathrm{fe}_{\mathrm{n}} \mathrm{g}_{\mathrm{n}=1}^{1}$ es un sistema ortonormal de $\mathrm{h}, \mathrm{y}$ los $\overbrace{\mathrm{f}}, 0$ con la propiedad de que

$$
{ }_{n=1}^{\lambda} A_{\bar{n}}<1:
$$

Lo que vamos a demostrar aquí, es $\operatorname{tr}\left(\Omega_{\mathrm{t}} \mathrm{a}\right){ }_{\mathrm{t}} !_{0} \operatorname{tr}(\Omega \mathrm{a})$ :

Es claro que,

$$
\operatorname{tr}\left(\Omega T_{\mathrm{t}} \mathrm{a}\right)={ }_{\mathrm{n}=1}^{\mathrm{X}} \mathrm{t}_{\mathrm{F}} \mathrm{hT}_{\mathrm{t}} \mathrm{ae}_{\mathrm{n}} ; \mathrm{e}_{\mathrm{n}} \mathrm{i} \quad \text { ( por la Prop.1.1.2) : }
$$

Pero, para todo $\mathrm{n} 2 \mathrm{~N}$;

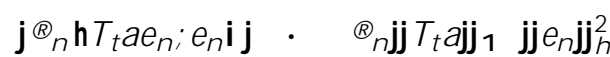

$$
\begin{aligned}
& =\epsilon_{4} j j P_{t}^{a} a P_{t} j_{1} \cdot \epsilon_{F} j j a j j_{1}
\end{aligned}
$$

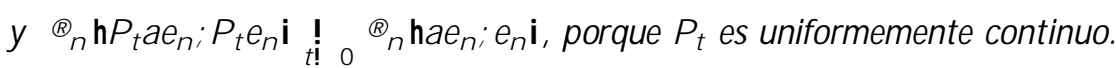
Entonces, tenemos que por el Teorema de Convergencia Dominada de Lebesgue,

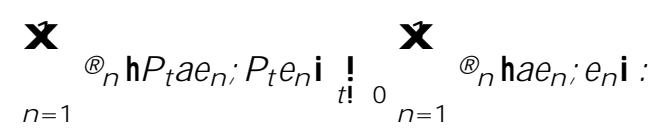

Pero como,

$$
\begin{aligned}
& x_{n=1}^{*} \epsilon_{m} h a e_{n} ; e_{n} i=x_{m=1}^{*} x_{n=1}^{*} A_{m} j e_{n} i h_{n} j a e_{m} ; e_{m}+
\end{aligned}
$$

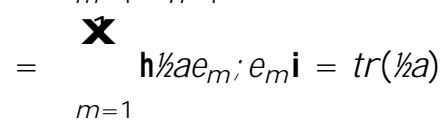


por lo tanto $\operatorname{tr}\left(\Omega_{\mathrm{t}} \mathrm{a}\right) \underset{\mathrm{t}}{\vdots} \mathrm{o}_{0} \operatorname{tr}(\Omega \mathrm{a})$ :

De la continuidad uniforme de $\mathrm{P}_{\mathrm{t}}$, se tiene que para todo a $2 \mathrm{~B}(\mathrm{~h})$ con jj a jj $j_{1}=1$;

$$
\begin{aligned}
& \mathrm{jjT}_{\mathrm{t}} \mathrm{a}_{\mathrm{i}} \mathrm{ajj}_{1}=\mathrm{jjP}_{\mathrm{t}}^{\mathrm{a}} \mathrm{aP} \mathrm{P}_{\mathrm{t}} \mathrm{i} \quad \mathrm{ajj}_{1} \\
& =j j P_{t}^{x} a P_{t} i a P_{t}+a P_{t} i a j j_{1} \\
& =j j\left(P_{t}^{\alpha} a_{i} a\right) P_{t}+a\left(P_{t} i \quad l\right) j j_{1} \\
& \text { - } \quad j j\left(P_{t}^{d} a_{i} a\right) P_{t} j j_{1}+j j a\left(P_{t} \quad l \quad l j j_{1}\right.
\end{aligned}
$$

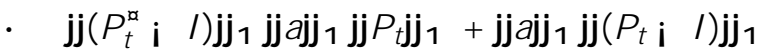

$$
\begin{aligned}
& \text { - } \quad j j\left(P_{t}^{\alpha} i \quad l\right) j j_{1}+j j\left(P_{t} i \quad l\right) j j_{1} \\
& =2 j j\left(P_{t} i \quad I\right) j j_{B(h)}:
\end{aligned}
$$

Ahora $j j T_{t}$ i I $j_{B} j_{B(B))}=\operatorname{Sup}_{a 2 B(h) ; j j a j j=1} j j T_{t} a_{i} \quad a j j_{1} \cdot 2 j j\left(P_{t} \quad I\right) j j_{1}$; así se tiene que $j \mathrm{jJ} \mathrm{T}_{\mathrm{t}} \mathrm{i} \quad \mathrm{Ij}_{\mathrm{B}(\mathrm{B}(\mathrm{h}))}{ }_{\mathrm{t}}{ }_{0} \quad 0$ : De donde se deduce que $T_{\mathrm{t}}$ es uniformemente continuo para todo $t, 0$.

Ahora calculamos \$ (a) :

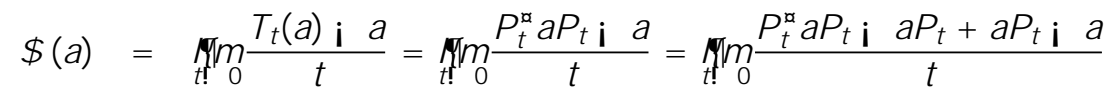

$$
\begin{aligned}
& =\lim _{t !} \frac{\left(P_{t}^{\alpha} i \quad\right) a P_{t}+a\left(P_{t} i \quad\right)}{t}
\end{aligned}
$$

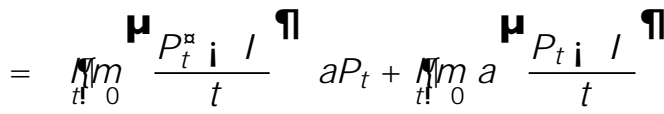

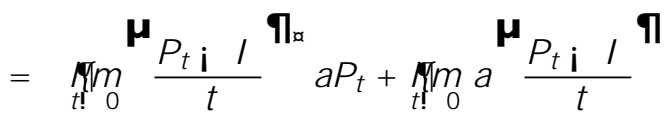

$$
\begin{aligned}
& =\lim _{t !} \frac{P_{t} i \mid}{t} \lim _{t !} \operatorname{lm}_{0} a P_{t}+\lim _{t !} m_{0} a^{\mu}{\frac{P_{t} i}{t}}^{\text {I }} \\
& =\mathrm{G}^{\mathrm{x}} \mathrm{a}+\mathrm{aG}:
\end{aligned}
$$

Por lo tanto $\$(a)=G^{\infty} a+a G$ : 
Ejemplo 5.1.2 ([9] , pág.198)Sea h espacio de Hilbert .

$$
T_{t}(a)=e^{i t i H} a e^{t i H}
$$

con $\mathrm{H}$ autoadjunto, es decir $\mathrm{H}=\mathrm{H}^{x}$ y acotado en $\mathrm{h}$.

Entonces $T_{t}$ en $B(h)$ es un semigrupo dinámico cuántico con generador in..: nitesimal

$$
\$(\mathrm{a})=\mathrm{i} \mathrm{i}[\mathrm{H} ; \mathrm{a}]:
$$

Como se trata de un caso particular del ejemplo anterior, con $\mathrm{G}=\mathrm{iH}$; entonces

$$
\begin{aligned}
\$(\mathrm{a}) & =\mathrm{G}^{\infty} a+a G \\
& =(\mathrm{iH})^{\infty}+a(i H) \\
& =i \mathrm{iH} a+a i H \\
& =i \mathrm{i}[\mathrm{H} ; \mathrm{a}]:
\end{aligned}
$$

Ejemplo 5.1 .3 ([8], págs.58; 59)El Semigrupo asociado al proceso de nacimiento puro.

Sea

$$
h=I_{2}{ }^{3} N^{[} f 0 g=f_{i n} g_{n} 1 / 2 C:{ }_{n}^{X} j i n j^{2}<1
$$

y $\mathrm{f} \Pi_{\mathrm{h}} \mathrm{g}_{\mathrm{n}} 1 / 2 \mathrm{C}$ tal que $\Pi_{\mathrm{h}} \in 0$ para todo $\mathrm{n} 2 \mathrm{~N}$.

Ahora sean $\mathrm{S}$ y $\mathrm{f}$ operadores en $\mathrm{h}$; de..nidos por

$$
\begin{aligned}
& D(f)=x^{\left(2 h:{ }_{n}^{X} j \Gamma j^{2} j \int n j^{2}<1\right.} \\
& f\left(\int\right)={ }_{n}^{X} \Pi h \int_{n} e_{n}=\left(\Pi \iint_{0}, \Pi \mathrm{h} \int_{1} ;:::\right) \\
& S\left(\int\right)=S\left(\int_{0} ; \int_{1} ;:::\right)=\left(0 ; \int_{0} ; \int_{1} ;:::\right) ; \\
& G=i \frac{j f j^{2}}{2} \text { : }
\end{aligned}
$$

Tomando ahora $\mathrm{L}_{1}=\mathrm{S} \pm f$; $\mathrm{L} \cdot=0$; operador cero para ` , 2; claramente tenemos que

$$
h G_{i ; i} i+h_{i} ; G_{i} i+h_{1} ; L_{1} i=0
$$

es decir se satisface la ecuación de la Hipótesis AA, además $D(G) 1 / 2 D\left(L_{1}\right)$ y para todo $i 2 \mathrm{~h}$ se cumple que

$$
\$\left(m\left(\int\right)\right)=m\left(A\left(\int\right)\right)
$$

con $\int 2 I_{1}$ ( $\mathrm{N} \mathrm{U} \mathrm{f0g);} \mathrm{el} \mathrm{álgebra} \mathrm{de} \mathrm{las} \mathrm{sucesiones} \mathrm{complejas} \mathrm{acotadas,} \mathrm{m}\left(\int\right)$ es el operador de multiplicación por $\int$; A es el generador in..nitesimal del proceso de nacimiento clásico actuando de la siguiente manera,

$$
A\left(\int\right)_{n}=j \Gamma j^{2}\left(\int_{n+1} i \int_{n}\right):
$$

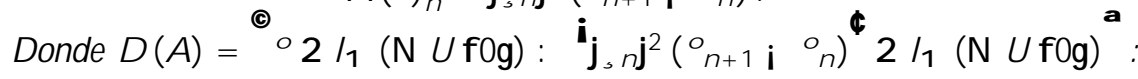


La forma cuadrática de $\mathrm{Q}_{1}$ satisface

$$
h_{m} ; Q_{1}(1) e_{n} i=\begin{aligned}
& 8 \\
& \gtrless
\end{aligned} 0 \text { si } n \in m \stackrel{9}{\geq}
$$

y por inducción se demuestra que

$$
e_{m} ; Q_{1}^{k}(1) e_{m}^{\circledR}={ }_{j=0}^{k} \frac{j \Pi_{m+j} j^{2}}{1+j \Pi_{m+j} j^{2}}:
$$

De acuerdo con el Teorema 3.2.7, el S.D.C., correspondiente es conservativo si y sólo si $Q_{1}^{k}(1){ }_{n} ! \quad 10$ y esto sucede si y sólo si

$$
\sum_{j=0}^{x} \frac{1}{j \Gamma h+j j^{2}}=1
$$

Ejemplo 5.1 .4 ([8]; pág.87)Un semigrupo dinámico cuántico minimal asociado al proceso de nacimiento y muerte con intensidades lineales.

Sea

$$
h=I_{2}{ }^{3} N^{[} f \circ g=f_{i n} g_{n} 1 / 2 C:{ }_{n}^{X} j i_{n} j^{2}<1
$$

y $\mathrm{N} ; \mathrm{a}^{+}$; a; los operadores de número, creación y aniquilación en $\mathrm{h}$; de..nidos mediante la siguiente regla de correspondencia:

$$
\begin{aligned}
a^{+}(e) & =p \overline{j+1} e_{+1} \\
a(e) & =j \bar{j} e_{i 1} \\
N\left(e_{g}\right) & =j e_{j}=a^{+} a(e) ;
\end{aligned}
$$

donde $f$ e $g_{j}$ es una base ortonormal de $h$;sus respectivos dominios son:

$$
\begin{aligned}
& D^{i} a^{+}=\underset{\left(2 h:_{n=0}^{x}(n+1) j i n j^{2}<1\right)}{\left(x^{x}\right.} \\
& D(a)=i 2 h:^{X} n_{j} j_{n_{i}} j^{2}<1 \\
& D^{i} a^{+} a^{\phi}=D(N)=i 2 h:_{n=0}^{k} n^{2} j i n j^{2}<1
\end{aligned}
$$

donde $f e_{j} g_{j}$ es la base canónica de $h$ : 
Sea $\$(x)$ con $x 2$ B (h) igual a

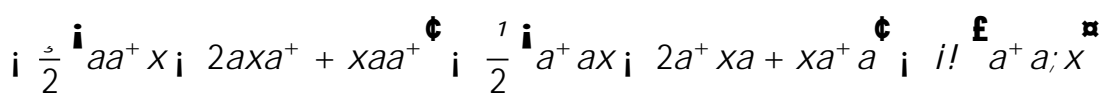

y dominio $D(N), \Pi \pi ; ! 2 R ; \Pi \pi, 0: S i !=0$ entonces este ejemplo corresponde a una extensión cuántica del semigrupo asociado al proceso de nacimiento y muerte con intensidades lineales. Ahora para $\$(x)$ de..namos los siguientes operadores $G ; L_{1}$ y $L_{2}$ de la siguiente manera:

$$
\begin{aligned}
G & =i \frac{\Pi}{2}(N+1) i \frac{\pi}{2} N+i ! N \\
L_{1} & =p \frac{\pi}{\Pi a^{+}} \\
L_{2} & =p \frac{\bar{\pi} a}{}
\end{aligned}
$$

con

$$
\left.D(G)=u 2 h:{ }_{n}^{x} j i \frac{\Pi}{2}(n+1) i \frac{\pi}{2} n+i ! n u_{n} j^{2}<1\right)
$$

de esta forma podemos escribir a $\$(x)$ como

A hora tomemos

$$
\$(x)=G^{\infty} x+x G+P_{?=1}^{2} L^{\not x} x L:
$$

$$
D_{1}=f u 2 h: 9 n_{0} \text { y } u_{n}=08 n>n_{0} g
$$

claramente se tiene que $D_{1}$ es un subespacio denso de $h$. Pero como $D\left(L_{1}\right)=$ $D\left(a^{+}\right) ; D\left(L_{2}\right)=D(a)$ y $D\left(a^{+}\right)=D(a)$ se tiene que $D(G) 1 / 2 D\left(L^{\prime}\right)$ para $\because=1 ; 2$ : Nos bastará trabajar todo este ejemplo sobre $D_{1}$, pues siendo este denso en $h$ y esencia de $a ; a^{+} y$ G; todo lo que se haga aquí con los operadores arriba mencionados lo mismo se seguirá valiendo sobre $h$. Vamos a demostrar que se satisface la ecuación de la Hipótesis AA, tomemos e; ; $e_{k} 2 D_{1}$ básicos: Entonces tenemos lo siguiente:

$$
\begin{aligned}
& h \mathrm{he}_{j} \mathrm{e}_{\mathrm{k}} \mathrm{i}+\mathrm{he} ; \mathrm{Ge}_{\mathrm{k}} \mathrm{i}+\mathrm{hL}_{1} \mathrm{e} ; \mathrm{L}_{1} \mathrm{e}_{\mathrm{i}} \mathrm{i}+\mathrm{hL}_{2} \mathrm{e}_{j} ; \mathrm{L}_{2} \mathrm{e}_{k} \mathrm{i}
\end{aligned}
$$

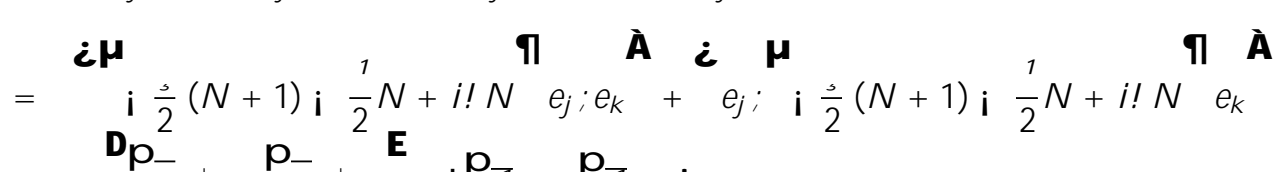

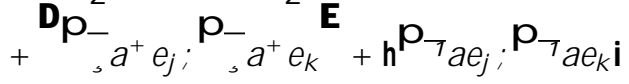

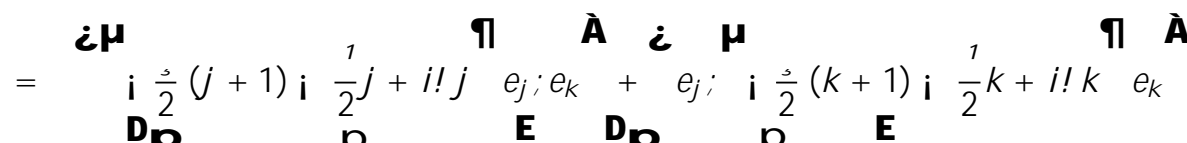

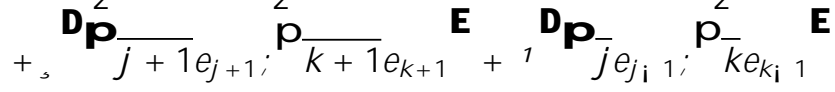

$$
\begin{aligned}
& =\Pi^{p} \overline{j+1}^{p} \overline{k+1}+\pi^{p} \bar{j}^{p} \bar{k}_{i} \Pi(j+1) i \pi j \\
& =0 \text {, si j = k: }
\end{aligned}
$$


es decir

$$
h_{G e} ; e_{k} i+h_{g} ; G_{k} i+{ }_{=1}^{X^{2}} h \cdot e_{j} ; L \cdot e_{k} i=0
$$

el cual es la propia ecuación de la Hipótesis A A, y como cualquier otro elemento del espacio es combinación lineal de estos básicos entonces tenemos que esta ecuación es válida en todo el espacio. G genera al semigrupo de contracciones $f P_{t} g_{t, 0}$ dadas por la siguiente regla

$$
P_{t}(\underline{a})_{n}=e^{\left(i \frac{\pi}{2}(n+1) i \frac{\pi}{2} n+i ! n\right) t \underline{a}_{n}}
$$

donde a $2 \mathrm{D}(\mathrm{G})$ : Si el lector no se convence de esto puede intentar calcular

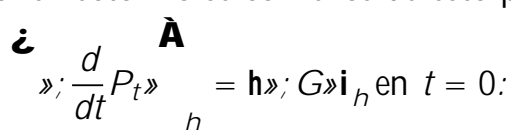

3) Ahora sea i el operador en $D_{1}$ de.nido por

$$
\mathrm{i}=\mathrm{L}_{1}^{\mathrm{x}} \mathrm{L}_{1}+\mathrm{L}_{2}^{\mathrm{x}} \mathrm{L}_{2} \text { : }
$$

Vamos a demostrar que i es esencialmente autoadjunto, es decir $\operatorname{Ker}\left(i^{\text {a }} \S i\right)=f 0 g$ :

Sea $i 2 \mathrm{Ker}\left(\mathrm{i}^{\alpha}+\mathrm{i}\right)$ entonces

$$
\mathrm{h} f ;\left(\mathrm{i}^{\alpha}+\mathrm{i}\right) \dot{\mathrm{i}}=0 \text {, para todo } \int 2 \mathrm{D}_{1}
$$

en general para los elementos de la base resulta que ha $;\left(i^{{ }^{\alpha}}+i\right) i \mathrm{i}=0$ : Con lo cual se tiene también lo siguiente:

$$
\begin{aligned}
& H_{i} i \text { i) } e_{i} i \mathrm{i}=0 \\
& h\left(\left[L_{1}^{x} L_{1}+L_{2}^{x} L_{2}\right] ; \quad i\right) e_{j} i \dot{i}=h(\Pi(j+1)+\pi j ; i) e_{j} ; i \\
& =\Pi(j+1)+\pi j i \text { ihe } ; i j \\
& =0 \text {; }
\end{aligned}
$$

lo cual implica que $i$ es ortogonal al subespacio generado por $f e g_{j}$; pero esto quiere decir que $i=0$; análogamente se hace para $\operatorname{Ker}\left(i^{{ }^{\alpha}} i \mathrm{i}\right)=\mathrm{f} 0 \mathrm{~g}$ : Por lo tanto tenemos que $\mathrm{K}$ er $\left(i^{\text {风 }} \S \mathrm{i}\right)=\mathrm{f} 0 \mathrm{~g}$ :

4) Ahora de..nimos el operador $C$ en $h$; de la siguiente forma

$$
\left.D(C)=u 2 h:{ }^{X} i_{\cdot 2}+1^{\Phi} j u \cdot j^{2}<1\right)
$$

con

$$
\mathrm{C}=\mathrm{CN}
$$

donde $\mathrm{N}$ es el operador de número y $c>2(\Pi+\pi)$ :

Claramente se tiene que $C^{\mathfrak{a}}=C$ y además $D_{1}$ es una esencia para $C$; pues este 
es multiplo del operador de número $\mathrm{N}$ :

Veremos que se satisface hu; i ui · hu; Cui :Tómese u $2 D_{1}$

$$
\begin{aligned}
& \text { hu; ; ui = hu; }\left(L_{1}^{x} L_{1}+L_{2}^{x} L_{2}\right) u_{\varnothing} i_{\circledast} \\
& =u_{i} \operatorname{laa}^{+}+\pi a_{\phi}^{+} a^{4} \text { u } \\
& =u^{u ; \Pi^{i} a^{+} a+1^{\phi}+\pi a^{+} a^{\alpha u}} \\
& ={ }^{X} \dot{u}_{k}[\Pi(k+1)+\pi k] u_{k} \\
& =(\Pi+\pi)^{x} k j u_{k} j^{2}+{ }^{X} \Pi u_{k} j^{2} \\
& \text {. } \quad(\Pi+\pi)^{X^{k}} k j u_{k} j^{2}+(\Pi+\pi)^{\text {X }} k j u_{k} j^{2} \\
& =2(\Pi+\pi)^{k} k j u_{k} j^{2} \\
& c^{X} k^{k j} u_{k} j^{2}=h u ; C u i \text { : }
\end{aligned}
$$

Así hemos probado lo que se había planteado y por lo tanto el semigrupo dinámico cuántico minimal asociado a los operadores $G$; $L_{1}$ y $L_{2}$ es conservativo.

Ejemplo 5.1 .5 ([7];págs;361 i 367) El Semigrupo asociado a la martingala de Azéma.

Ahora en este ejemplo tomemos $a h=L^{2}(R, d x)$ y los $P_{t}$; para todo $t, 0$, en $B(h)$ de..nidos por

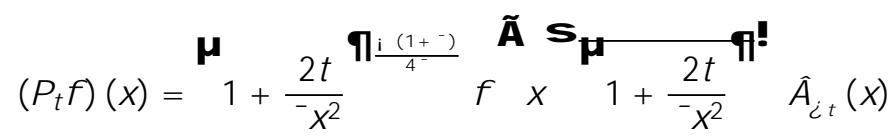

donde $\times 2 R, x \in 0 ; \varnothing \in 0$,

$$
\varnothing_{t}={ }^{1 / 2} \times 2 R: x^{2}, i \frac{2 t^{3 / 4}}{\varnothing},
$$

con $\neg_{\varnothing_{t}}$ función indicadora de $\varnothing_{t}$ : Es fácil veri..car a partir de $\mathrm{P}_{\mathrm{t}}$; que

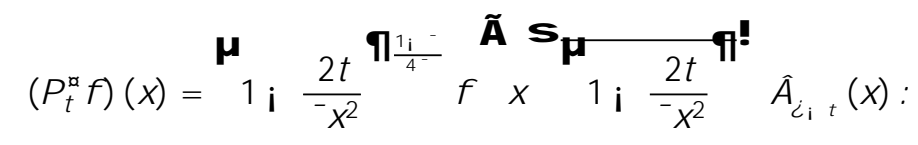

Sea $\mathrm{D}$ el espacio de todos los $\mathrm{f} 2 \mathrm{~h}$ tales que son in..nitamente diferenciables, con soportes compacto y que se anulan en una vecindad del 0: El operador $\mathrm{G}$ con dominio $\mathrm{D}$ dado por la siguiente expresión se extiende al generador 
in..nitesimal del semigrupo de contracciones en $\mathrm{h}$ dado por $\mathrm{P}_{\mathrm{t}}$; [7]:

$$
\begin{aligned}
(G f)(x) & =\frac{f q(x)}{\varnothing x} i \frac{1+\varnothing}{2 \varnothing^{2} x^{2}} f(x) \\
\left(G^{x} f\right)(x) & =i \frac{f \|(x)}{\varnothing x}+\frac{\varnothing_{i} 1}{2 \varnothing^{2} x^{2}} f(x):
\end{aligned}
$$

Considérese el operador $\mathrm{M}$ en $\mathrm{h}$ de..nido por

$$
D(M)={ }^{1 / 2} u 2 h: \frac{u(x)}{x} 2 h^{3 / 4} \text { y }(M u)(x)=\frac{1}{\varnothing x} u(x):
$$

Sea $\mathrm{S}$ operador unitario en $\mathrm{h}$ dado por

$$
\text { (Su) }(x)=p \frac{1}{\overline{j c j}} u^{3} \frac{x^{\prime}}{c} ;
$$

donde $c=1+\varnothing$ con $\varnothing 2 \mathrm{R}$; f0; i $1 \mathrm{~g}$ y $L_{1}=S \pm M, L \cdot=0$ el operador cero para ', 2:

Ahora sea \$ en D; dado por

$$
\$(f)(x)={\frac{\mu}{\varnothing^{2} x^{2}}}^{i} f(c x) \text { i } f(x) \text { i } \varnothing x f^{q}(x)^{\Phi^{\text {ी }}}
$$

con $\mathrm{f}$ acotada y derivable. Se cumple también que

$$
\begin{aligned}
h \int(x) ; \$(1) i(x) i= & h G^{\alpha} \int(x) ; i(x) i+h(S \pm M) \int(x) ;(S \pm M) i(x) i \\
& +h \int(x) ; G i(x) i \\
= & 0
\end{aligned}
$$

por lo que se veri..ca la ecuación de la Hipótesis AA. Ahora para estudiar la conservatividad del S.D.C. minimal asociado; calculemos los operadores $Q_{\Pi}$ para todo П, 0( [12] Lema 2;1, pág.361 i 367); $Q_{1}$ viene dado por los siguientes casos,

a) si $\varnothing<0$

$$
\left(Q_{1} f\right)(x)=E e^{\frac{1}{2} \not x^{2}{ }_{a}}{ }^{3} c x^{p} \overline{1+\underline{a}}
$$

donde a es una variable aleatoria positiva con función de distribución

$$
P(\underline{a} \cdot s)=1 i(1+s)^{\frac{1}{2 \varnothing}}
$$

con S, 0; siendo $\mathrm{E}$ la función esperanza matemática.

b) Si $\varnothing>0$

$$
\left(Q_{1} f\right)(x)=E e^{i \frac{1}{2} \phi x^{2 a} f^{3}} c x^{p} \overline{1 i^{\underline{a}}}
$$


donde a es un variable aleatoria con valores en $(0 ; 1)$ y su función de distribución es dada por

$$
P(\underline{a} \cdot s)=1 ;(1 ; s)^{\frac{1}{2 \varnothing}} ; \quad \operatorname{con} 0 \cdot s \cdot 1 .
$$

Por inducción se tiene también que para $\mathrm{f}_{\mathrm{a}_{i}} \mathrm{~g}_{\mathrm{i}}$ sucesión de variables aleatorias independientes positivas e identicamente distribuidas en $R$, con distribución por $(5 ; 1)$ ó $(5 ; 2) \mathrm{Q}_{1}^{\mathrm{n}}(1)$; para cada caso viene dado por

a) $\varnothing<0$

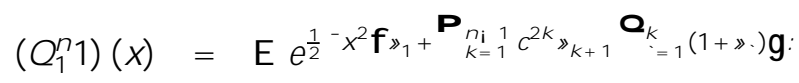

b) $\varnothing>0$

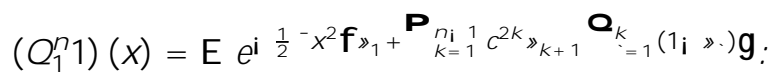

Ahora el paso crucial es la conservatividad del semigrupo dinámico cuántico minimal asociado a todas estas condiciones, para ello se hace uso de dos resultados importantes de [12] Teorema 2.3.1 y Teorema 2;3;2, págs.37 i 38. A nalicemos el caso $\varnothing<0$ : Por lo tanto, sólo será necesario ver la convergencia o divergencia de la serie

$$
\sum_{k=1}^{x^{1}} c^{2 k} \underline{a}_{k+1}^{Y^{k}}\left(1+\underline{a}_{i}\right)
$$

el cual a la vez depende del parámetro $\mathrm{c}$ :

Si jcj, 1 entonces $\mathrm{j} 1+\varnothing$, 1 y por lo tanto $\varnothing$ · i 2 pues $\varnothing<0$; y además se tiene que

$$
\underline{a}_{k+1}^{Y^{k}} c^{2}\left(1+\underline{a}_{1}\right), \underline{a}_{k+1}
$$

pero siendo las variables $\underline{a}_{k+1}$ positivas, independientes e idénticamente distribuidas se sigue que la serie formada por

$$
c^{2 k \underline{a}_{k+1}}{ }_{=1}^{k}\left(1+\underline{a}_{\cdot}\right)
$$

diverge con probabilidad 1, obteniéndose así que el semigrupo minimal es conservativo.

Si jcj $<1$ entonces i $2<\varnothing<0$ y aplicamos [9] Lema 2;3;2; págs.38 i 39, que dice

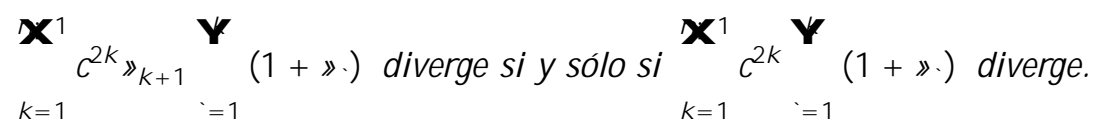


Tomando logaritmo natural y aplicando la Ley Fuerte de los Grandes Números a esta última serie obtenemos que

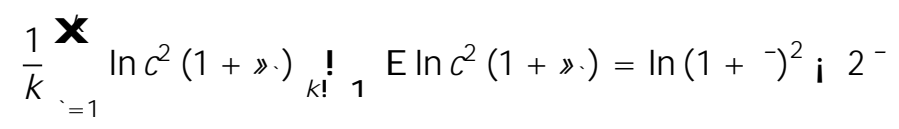

con probabilidad 1 , donde $\ln c^{2}\left(1+\underline{a}_{-}\right)$tiene distribución exponencial con parámetro $+\frac{1}{2 \varnothing}$ :

Considerando la ecuación $(1+\varnothing)^{2}$ e $2 \varnothing=1$; el cual tiene dos raices $\varnothing=0$ y $\varnothing^{\alpha}=$ i 1;278::: la cual es la única raíz negativa, analizamos los casos siguientes:

a) $\varnothing^{\alpha}<\varnothing$; tomemos $\varnothing^{\alpha}<$ i $1<\varnothing<0$ :

Derivando a $\ln (1+\varnothing)^{2}$ i $2 \varnothing$ obtenemos que

$$
{\frac{d^{3}}{d \varnothing}}^{3} \ln (1+\varnothing)^{2} i 2 \varnothing^{\prime}=i \frac{2 \varnothing}{(1+\varnothing)}:
$$

Claramente para todo $\varnothing 2(\mathrm{i} 1 ; 0)$ se tiene que $\ln (1+\varnothing)^{2}$ i $2 \varnothing$ es una función creciente. Pero como $\ln (1+0)^{2}$ i $2(0)=0$ entonces se tiene que $\ln (1+\varnothing)^{2}$ i $2 \varnothing<0$ : Por lo tanto se deduce que si $k$ ! 1

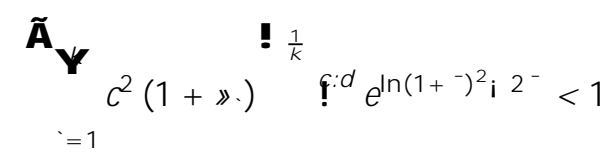

resultando por lo tanto que

$$
\mathrm{k}=1^{\mathrm{X}} \mathrm{c}^{2 \mathrm{k}} \mathrm{Y}^{\mathrm{k}}\left(1+\underline{a}_{1}\right)
$$

es convergente debido al criterio de la raíz, con lo cual el semigrupo minimal no es conservativo. Esto demuestra que la $\mathrm{H}$ ipótesis $\mathrm{A} A$ es necesaria pero no es su..ciente para la conservatividad.

b) Consideremos $\varnothing^{\alpha}<\varnothing<$ i 1 : A hora resulta que para todo $\varnothing 2\left(\varnothing^{\alpha} ;\right.$; 1$)$; $\ln (1+\varnothing)^{2}$; $2 \varnothing$ es una función decreciente y como $\ln \left(1+\varnothing^{\alpha}\right)^{2}$ i $2 \varnothing^{\alpha}=0$, tenemos otra vez que $\mathrm{e}^{\ln (1+\varnothing)^{2} i} 2 \varnothing<1$ y así

$$
\sum_{k=1}^{x} c^{2 k} Y_{=1}^{k}\left(1+\underline{a}_{1}\right)
$$

es convergente. 
c) A hora tomemos $\varnothing<\varnothing^{\alpha}<$ i 1 . Tenemos que $\ln (1+\varnothing)^{2}$ i $2 \varnothing$ es una función decreciente, de donde se deduce que $e^{\ln (1+\varnothing)^{2}}$ i $2 \varnothing>1$ : Lo cual implica que

$$
{ }_{k=1}^{X} c^{2 k} y_{=1}^{Y^{k}}(1+\underline{a} .)
$$

es divergente, resultando así que el semigrupo minimal es conservativo.

d) El caso crítico lo tenemos cuando $\varnothing=\varnothing^{\alpha}$ : Aquí se tiene que

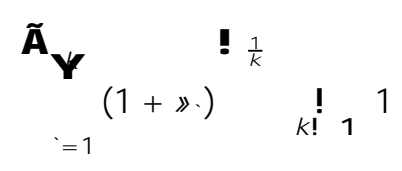

con probabilidad 1, lo cual en este caso el criterio de la raíz no nos proporciona información alguna sobre esta serie. Entonces consideremos la caminata aleatoria

$$
{ }^{(} X^{k} \ln c^{2}\left(1+a_{1}\right): K=1 ; 2 ;::
$$

donde $\ln c^{2}(1+\underline{a}$,$) son v.a.i.i.d, ` 1$ y $\ln (1+\underline{a}) ,2 L^{1}$ porque tiene distribución exponencial con parámetro $+\frac{1}{2 \varnothing}$ : Tenemos así lo siguiente

$$
E \ln c^{2}\left(1+\underline{a}_{1}\right)=\ln (1+\varnothing)+E \ln \left(1+\underline{a}_{-}\right)=\ln \left(1+\varnothing^{\alpha}\right) ; 2 \varnothing^{\alpha}=0 ;
$$

entonces resulta que la caminata aleatoria es recurrente ([4] Teorema 3;38, pág.56). Así que la serie

$$
x_{k=1}^{x} c^{k} c^{2}\left(1+a_{1}\right)
$$

es divergente casi seguramente por la ley 0-1 de Hewitt-Savage ([4], pág.63). Resultando así que el semigrupo minimal es conservativo.

A hora sólo nos resta ver qué pasa cuando $\varnothing>0$. Tomando el respectivo $Q_{1}^{n}(1)$ y las distribuciones dada por $(5 ; 2)$ obtenemos que $i \ln (1 ; \underline{a}$. $)$ tiene distribución exponencial con parámetro $\frac{1}{2 \varnothing}$ : A hora auxiliándonos de la función $f(\varnothing)=(1+\varnothing)$ ei $\varnothing$; claramente se tiene quef $(\varnothing)>0$ para todo $\varnothing, 0$ y a la vez $f(\varnothing)$ es decreciente. Por lo tanto se tiene que $0<f(\varnothing) \cdot 1$ es decir, $f$ alcanza su máximo en $\varnothing=0$ y además

$$
\ln f(\varnothing)^{2}=\ln (1+\varnothing)^{2} \text { i } 2 \varnothing \text {. }
$$

Denotemos por

$$
\mathrm{i} \cdot=\mathrm{c}^{2}\left(1 ; \mathrm{a}_{1}\right) \text {; }
$$


entonces tenemos que

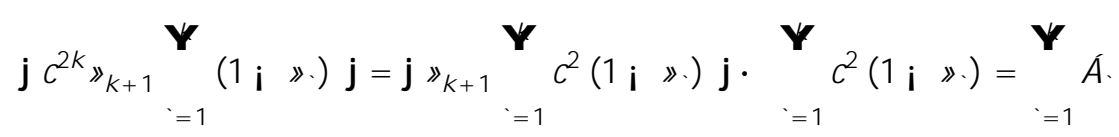

con lo cual, al aplicar el criterio de la raíz, tomando logaritmo natural y aplicando la ley de los grandes números se tiene lo siguiente

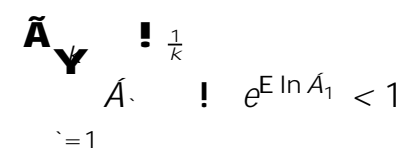

pues

$$
\begin{aligned}
& E \ln ; .=E \ln c^{2}\left(1 ; \quad a_{-}\right)=\ln (1+\varnothing)^{2}+E \ln \left(1 ; \quad a_{-}\right) \\
& =\ln (1+\varnothing)^{2} \text { i } 2 \varnothing=\ln ^{2}(\varnothing)<0
\end{aligned}
$$

Por lo tanto concluimos que

$$
\begin{gathered}
X^{k} \\
k=1 \\
;
\end{gathered}
$$

converge con probabilidad 1 , y por consiguiente se tiene que

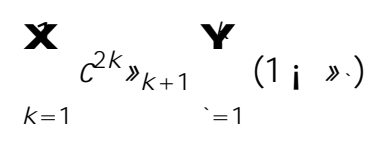

converge con probabilidad 1, resultando así que el semigrupo minimal no es conservativo. 


\section{B ibliografía}

[1] Bartle R. G., The Elements of Real A nalysis. Second Edition 1976 (J hon Willey \& Sons).

[2] Berberian S. K., Lectures in Functional Analysis and Operators Theory,1998 (Springer-Verlag. New Y ork).

[3] B ratteli O. and Robinson D.W., O perators A lgebras and Quantum Statistical Mechanics 1 . Texts and M onograph in Physics.

[4] B reiman Leo., Probability. Addison-Wesley Publishing Company.1968.

[5] Chevotarev A. M., Lectures on Quantum Probability.Aportaciones M atemáticas, 2000 (SM M).

[6] Chevotarev A. M. and Fagnola F., Suф cient Conditions for Conservativity of Minimal Quantum Dynamical Semigroups. J. Funct. A nal.118(1993).

Págs.131-135.

[7] Chevotarev A. M. and Fagnola F., On Quantum Extensions of the A zéma Martingale Semigroup, Sém. Prob. XXIII (1989) ; págs.67-68.

[8] Fagnola F., Quantum Markov Semigroups and Quantum Flows. Proyecciones Revista Matemática. 1991. $\mathrm{N}^{ \pm} 3$.

[9] Fagnola F and Rebolledo R., Stochastic Partial Dixerential Equations and A plications, págs.197-207 (M arcel Decker, Inc.) 2002.

[10] García J. C., Una clase de transformaciones completamente positivas no acotadas y conservatividad de la solución minimal de la ecuación maestra. (Tesis doctoral).

[11] García J. C and Quezada Roberto., Conditions for Nonconservativity in Quantum Dynamical Semigroups. Contemporary Mathematics. 136; 161 i 169; 2003: 
[12] García J . C and Quezada Roberto., Hille-Y osida estimate and nonconservativity of quantum dyanamical semigroups, to appear.

[13] García J. C., Divergence criteria for Series related to the Azéma-E mery Martingale process, Mathematical Notes. Vol. 57, $\mathrm{N} \pm .4,1995$.

[14] K rauss K., General States Changes in Quantum Theory, 1971 (Ann. Phys. 64), págs. 311-335

[15] K ato T., P erturbation Theory for Linear O perators, 1966 (Springer-Verlag).

[16] Lamperti J ., Stochastic Processes, 1977 (Springer-Verlag. New York).

[17] M CBride A. C., Semigroups of Linear Operators, 1987 (Longman Scenti..CTechnical).

[18] Reed M. Simon., Methods of Modern Mathematical Physics, Vol. 1, Functional A nalysis, 1975 (A cademic-P ress).

[19] Parthasaraty K. R., An Introduction to Quantum Stochastic Calculus, 1992 (B irhäuser-Verlag).

[20] Pazy A., Semigroups of linear Operators and Aplication to Partial Dixerential Equations, 1983 (Springer-Verlag. New York).

[21] Stinespring W. P., Positive Functions on C*algebras, P roc. A mer. M ath. Soc., 1995, pág.211-216.

[22] Sunders V. S., An Invitations to von Newmann Algebras, 1986 (SpringerVerlag).

[23] V ladimir T kachuck., Topología General . U.A.M. I, A gosto 1999.

[24] Weidman J ., Linears Operators in Hilbert Spaces, 1980 (Springer-Verlag. New York).

[25] Yosida K ., Functional Analysis, 1980 (Springer). 